\title{
Estudo de Técnicas de Teste de Regressão Baseado em Mutação Seletiva
}

\author{
Luciana Andréia Fondazzi Martimiano
}

Orientador: Prof. Dr. José Carlos Maldonado

Dissertação apresentada ao Instituto de Ciências Matemáticas e de Computação - ICMC-USP, como parte dos requisitos para obtenção do título de Mestre em Ciências - Área: Ciências de Computação e Matemática Computacional. 
Ao Richard, aos meus pais, Darcy e Cândido, e aos meus irmãos, Fabiano e Renato, pelo incentivo e apoio dedicados. 


\section{Agradecimentos}

A Deus por me acompanhar em todos os momentos.

Ao meu orientador, Prof. Dr. José Carlos Maldonado, pelo esforço, dedicação, amizade e profissionalismo que sempre demonstrou durante todo periodo deste trabalho.

Ao Richard, que sempre me deu apoio mesmo estando longe e compreendeu todas as minhas ausências superando as saudades e problemas que tivemos.

A minha familia, que com paciência sempre compreendeu minhas ausências. Em especial, aos meus pais Darcy e Cândido e aos irmãos Fabiano e Renato, por acreditarem em mim, me apoiando e incentivando.

Em especial às Prof ${ }^{\text {sa }}$. Dr ${ }^{\mathrm{a}}$. Itana Gimenes e Sandra Ferrari pelo incentivo, apoio e carinho que sempre me deram.

Às minhas amigas Tatiana, Thelma e Valéria, por dividirem comigo não só o lar mas também momentos alegres e dificeis. Em especial às amigas Andrea e Nilda.

Aos amigos do Grupo de Engenharia de Software: Adenilso, André, Ana Paula, Delamaro, Ellen, Elisa, Plínio, Rosângela, Rurik, Sandra, Silvia, Vangrei e William, pelo apoio e pelos bons e divertidos momentos que passamos juntos. Em especial, ao Auri e à Simone, que me ajudaram muito no desenvolvimento deste trabalho.

Aos meus amigos: Araxá, Ariane, Alessandro, Adilson, Alessandra, Astor, Boni, Cláudia, Cláudio Alex, Chandler, Daniel, Dani Bola, Dudu, Elder, Emerson, Emesto, Gustavo, Jamaica, João, Júlio, Marcos, Leonardo, Léo, Ludimila, Mac, Matite, Maristela, Marisa, Meykell, Mirla, Monique, Nixon, Omar, Ornan, Paulo, Paulo Cardoso, Paulo Noronha, Pissioli, Rejane, Regina, Roberto, Rudinei, Simone, Silvia, Taboca, Tanão, Tavinho e Wagner, pelos bons momentos que passamos juntos que sempre estarão aguardados em meu coração.

À Beth, Laura, Marilia, Adriana e Sandra, pela disposição e atenção que sempre demonstraram.

A todos aqueles que, de certa forma, me apoiaram neste trabalho.

À Fapesp pelo apoio financeiro. 


\section{Resumo}

Independentemente do tipo de manutenção conduzida - corretiva, preventiva, adaptativa ou evolutiva, as atividades de teste de regressão são necessárias para testar as modificações realizadas $e$ as eventuais novas funcionalidades de um programa, e, principalmente, para testar se as funcionalidades já existentes não foram afetadas adversamente pelas modificações. Visando a aplicar o teste de regressão de uma maneira sistemática a um baixo custo e com eficácia, muitas técnicas têm sido propostas na literatura. Essas diversas técnicas são divididas em duas abordagens: retest-all e seletiva. A abordagem retest-all utiliza todo o conjunto de casos de teste disponivel para testar as modificações, enquanto que a abordagem seletiva utiliza um subconjunto dos casos de teste disponiveis para testar as modificações. As técnicas baseadas na abordagem seletiva têm sido muito estudadas, pois visam a diminuir os esforços despendidos no teste de regressão reduzindo o número de casos de teste a serem reexecutados. Diante da diversidade das técnicas seletivas, fazem-se necessários estudos empíricos para avaliar e comparar a aplicação dessas técnicas. Assim, este trabalho visa a aplicar e avaliar empiricamente duas técnicas de teste de regressão que têm se mostrado promissoras: a Técnica baseada em Modificação (Wong et al., 1997a) e a Técnica baseada em Mutação Seletiva (Wong et al., 1997b). Para auxiliar nessa avaliação, um framework, proposto por Rothermel e Harrold (1996), é utilizado. Com a realização desses experimentos, espera-se contribuir para o estabelecimento de estratégias de teste de regressão efetivas e de baixo custo. 


\section{Abstract}

Independently of the type of maintenance carried out - corrective, preventive, adaptative or perfective, regression testing activities are necessary to test the modifications and the new eventual features, and, mainly, to test if the features existent were not adversely affected by the modifications. Aiming at carrying out the regression testing systematically, with low cost and effectiveness, many techniques have been proposed in the literature. These techniques are divided into two approaches: retest-all and selective. The retest-all approach uses the complete test case set available; meanwhile, the selective approach selects a subset to carry out the regression testing. Many researchers have studied the techniques based on the selective approach, since they aim at reducing the efforts during the regression testing reducing the test case set to be reexecuted. As there are several techniques based on selective approach, empirical studies are necessary to evaluate and compare these techniques. Thus, this work aims at evaluating and comparing the application of two promising regression testing techniques: Technique based on Modification and Technique based on Selective Mutation. A framework, proposed by Rothermel and Harrold, is used to evaluate these techniques. The accomplishment of these studies contributes towards the establishment of effective and low cost regression testing strategies. 


\section{Sumário}

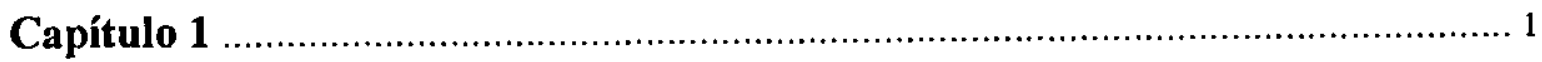

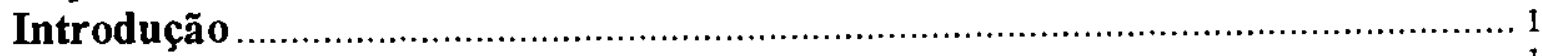

1.1 Contexto.

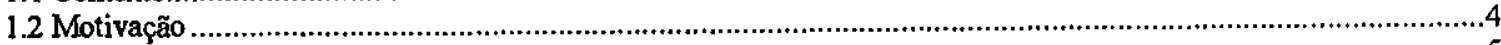

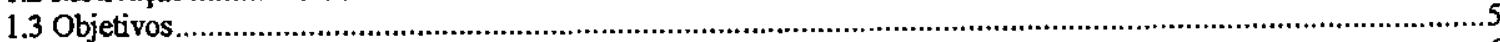

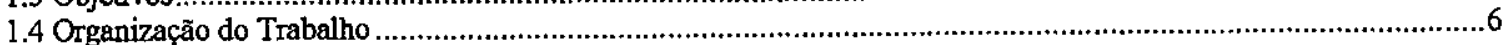

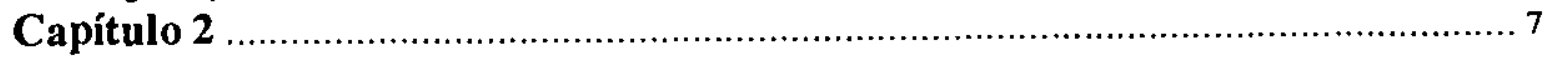

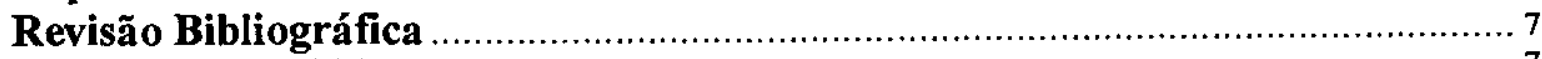

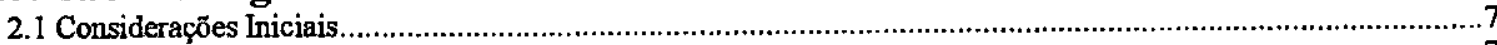

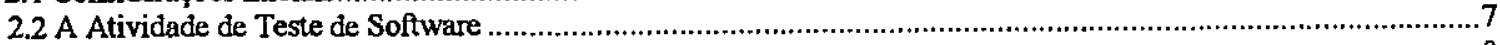

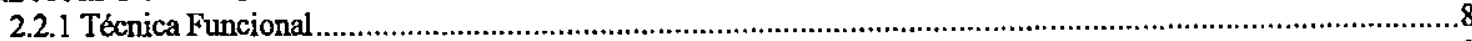

2.2.2 Técnica Estrutural .........................................................................................................................

2.2.3 Técnica Baseada em Erros.................................................................................................................10

2.3 A Atividade de Teste de Regressão ..........................................................................................................

2.3.1 Framework de Avaliação e Comparação de Técnicas de Teste de Regressão Seletivas...................................17

2.3.2 Um Modelo de Custo para Comparar Técnicas Seletivas e Técnicas Retest-all ................................................22

2.4 Técnica de Teste đe Regressão baseado em Modificą̧ão ....................................................................................26

2.5 Técnica de Teste de Regressão baseado em Mutação Seletiva ……………..........................................................30

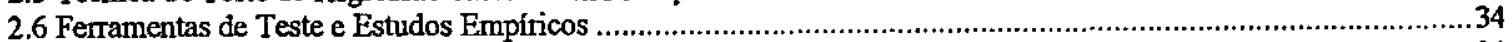

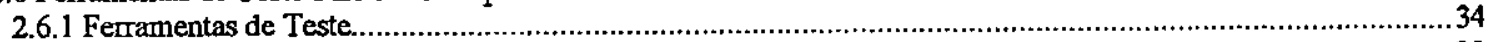

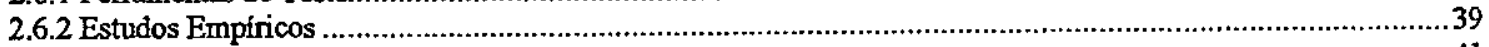

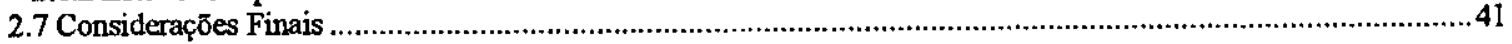

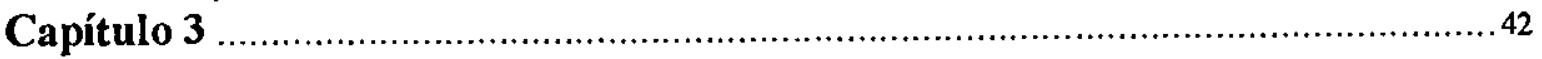

Aplicação e Avaliação das Técnicas de Teste de Regressão ..........................42

3.1 Considerą̧⿸丆⿰丨丶⿱一⿻上丨

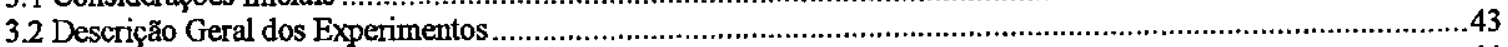

3.2.1 Aplicação e Avaliação da Técnica baseada em Modificação ........................................................................44

3.2.2 Aplicação e Avaliação da Técnica baseada em Mutação Seletiva ……….......................................................59

3.2.3 Análise Comparativa dos resultados obtidos com a Aplicação de ambas as Técnicas de Teste đe Regressão com

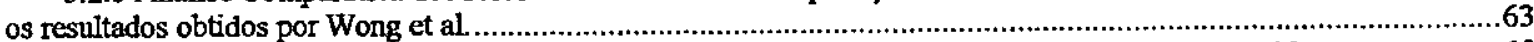

3.2.4 Avaliação das Técnicas de Teste de Regressão segundo o Framework de Rothermel e Harrold.........................65

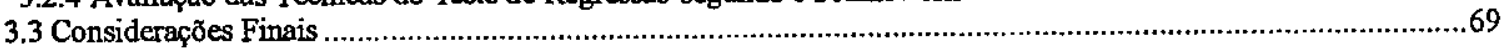

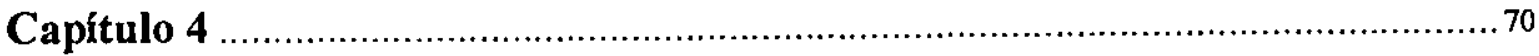

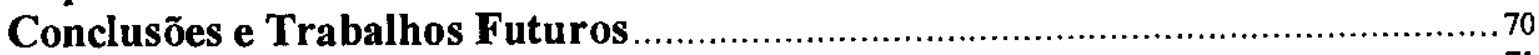

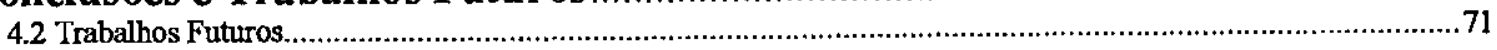

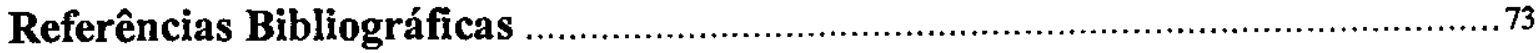

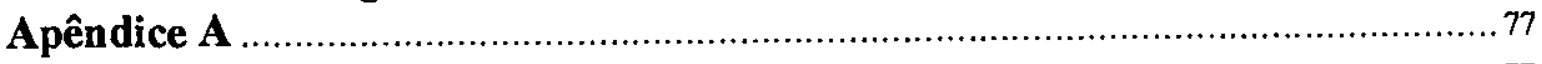

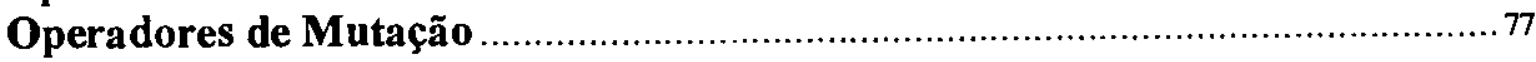

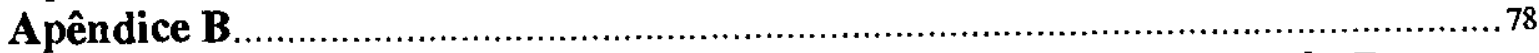

Versões Modificadas dos Programas UNIX e Ativação dos erros do Programa

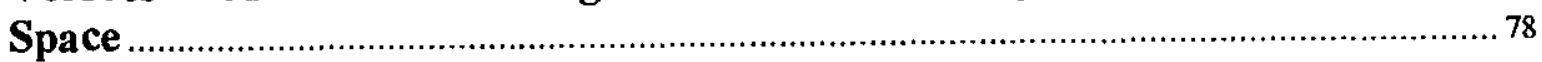




\section{Lista de Figuras}

Figura 1.1 - llustração das atividades realizadas.

Figura 2.2 - Conjunto T"' baseado em modifica çāo.

Figura 2.3 - Seleção dos casos de teste de regressão utilizando um processo off-line (Wong et al.,1997a).

Figura 2.4 - Redução do conjunto de teste de regressäo (Wong et al 1997b). ____ 31

Figura 3.1 - Relação entre as Conjuntos de Operadores de Mutação.

\section{Lista de Tabelas}

Tabela 2.1 - Lista de erros utilizada para gerar os programas com defeitos.

Tabela 2.2 - Programas UNLX utilizados nos experimentos.

Tabela 2.3 - Tipos de defeitos utilizados nos experintentos.

Tabela 3.1 - Dados dos programas UNIX.

Tabela 3.2 - Categorização dos defeitos dos programas UNDX —... 45

Tabela 3.3- Precisãa e eficácia do conjun

Tabela 3.4 - Conjun to T' para cada defeito.

Tabela 3.5 - Conjunto T"' para cada defeito._48

Tabela 3.6-Nümero de mutantes gerados por operador de mutação essencial __ 49

Tabela 3.7 - Nimero de casos de teste nos conjuntos minimizados T" MD _____ 49

Tabela 3.8 - Número de casos de teste nos conjuntos priorizados $T^{\prime \prime}$ "

Tabela 3.9-Média para os conjunbs minimizados T"

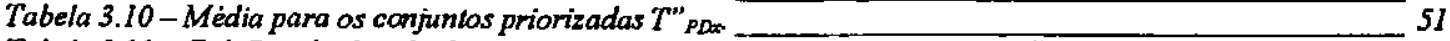

Tabela 3.11-Cal: Resultados obtidos para cada um das conjuntos minimizados $T^{\prime \prime}{ }_{M 0} \longrightarrow 53$

Tabela 3.12 - Cal: Resullados oblidos para cada um dos conjuntos priorizados $T$ "

Tabela 3.13 - Checkeq: Resultados ob tidos para cada um dos conjuntos minimizados T"

Tabela 3.14-Checkeq: Resultados oblidos para cada um dos conjuntos priorizados $T^{\prime \prime}{ }_{P D}$ ___ 55

Tabela 3.15-Comm: Resultados obtidos para cada um dos conjuntos minimizados T" ${ }_{M D x} \longrightarrow 56$

Tabela 3.16 - Comm: Resultados obtidos para cada um dos conjuntos priorizados $T$ " ${ }_{P D *} \longrightarrow 56$

Tabela 3.17-Loak: Resultados obtidos para cada um dos conjumlos minimizados T" MBx-— 57

Tabela 3.18 - Look: Resultados obtidos para cada um dos conjun los priorizados T"

Tabela 3.19-Uniq: Resultados obtidos para cada um dos conjuntos minimizados $T$ " Ma_______ 58

Tabela 3.20 - Uniq: Resultados obtidos para cada um dos conjuntos priorizados $T$ "

Tabela 3.21 - Dados do Programa SPACE.

Tabela 3.22 - Categorização dos Defeitos do Programa SPACE. ______...__ 60

Tabela 3.23 - Nümero de Casos de Teste que revelam os Defeilos do Programa SPACE. 60

Tabela 3.24-Conjunto de Operadores Essenciais e os Mutantes Gerados para cada Operador. ___ 61

Tabela 3.25-Total de Mutantes gerados para o Conjunto de Operadores de Mutaçäo defunidos por Wong. 61

Tabela 3.26-Reduçäo de Tamanho, Eficácia, Precisäo e Recall da conjunto T' selecionado. ___ 63

Tabela 3.27- Precisão e Recall do conjunto T' com relação a cada defeito.

Tabela 3.28 - Média para os conjuntos minimizados e priorizados dos programas UNL $X^{*}$. 64

Tabela 3.29-Número de casos de teste selecionados para os conjun los reduzidos com relação ao critério

MUT-A para o programa Cal.

Tabela 3.30 - Média dos resultados de redução de eficácia, inclusão e precisão_F para os conjuntos minimizados e priorizados do programas $U N D X^{*}$.

Tabela 3.31-Média dos resultados de inclusão e precisão_F para o programa SPACE. 


\section{Lista de Quadros}

Quadro 2.1 - Programas que compöem a PROTEUMAM e a PROTEUM.

Quadro 2.2 - Funçðes implementadas pela ferramenta POKE-TOOL.

Quadro A.1 - Operadores de Mutação utilizados por Wong et al. 


\subsection{Contexto}

Com a crescente demanda de software e a conseqüente evolução da Engenharia de Software, atividades agregadas sob o nome de Garantia de Qualidade de Software têm sido introduzidas ao longo de todo o processo de desenvolvimento, entre elas as atividades de VV\&T - Verificação, Validação e Teste, com o intuito de auxiliar na melhoria da qualidade e da produtividade.

As atividades de teste envolvem basicamente quatro etapas: planejamento de testes, projeto de casos de teste, execução e avaliação dos resultados (Myers, 1979; Biezer, 1990; Maldonado, 1991; Pressman, 1992). Essas atividades são realizadas em três diferentes níveis: nível de unidade, nível de integração e nível de sistema. Geralmente, os critérios de teste de software são estabelecidos a partir de três técnicas: funcional, estrutural e baseada em erros. Cada uma dessas técnicas estabelece os requisitos de teste a partir de diferentes aspectos do software. A técnica funcional estabelece os requisitos de teste a partir da especificação do programa. A técnica estrutural estabelece os requisitos a partir da implementação do programa, enquanto que a técnica baseada em erros estabelece os requisitos de teste a partir de erros típicos cometidos no processo de desenvolvimento de software. Independentemente da técnica utilizada, um ponto importante que se considera em diversos estudos empíricos é a redução dos custos aplicados às atividades de teste. Além da redução dos custos, a eficácia também é um dos aspectos fundamentais bastante investigado (Mathur e Wong, 1993; Wong, 1993; Mathur e Wong, 1994; Wong et al., 1994a; Wong et al., 1994b; Offut et al., 1996a; Offut et al., 1996b).

Observa-se que o conjunto de informações oriundas das atividades de teste pode ser utilizado no contexto das atividades de depuração, estimativa de confiabilidade e de manutenção 
(evolução) de software (Ostrand e Weyuker, 1988; Hartmann e Robson, 1990; Pressman, 1992; Varadan, 1995).

Independentemente da qualidade da concepção, desenvolvimento e teste do sistema antes de ter sido liberado, o produto de software irá certamente ser modificado por diversas razões, por exemplo para atender às mudanças nas especificações e expectativas dos usuários (Lehman, 1996, Wong et al., 1997a, Wong et al., 1997b). Assim, a fase de manutenção é a etapa do ciclo de vida do software na qual são efetuadas alterações no produto após sua liberação para o usuário. Qualquer que seja o tipo de manutenção - corretiva, evolutiva, adaptativa ou preventiva - algumas tarefas comuns devem ser efetuadas: o entendimento, a modificação e a revalidação do software. Durante a etapa de revalidação deve-se demonstrar que não somente a nova lógica está correta, mas também que as partes do software que não foram modificadas permanecem intactas e o software como um todo ainda funciona corretamente (Pressman, 1992).

A atividade de teste da evolução do sistema é geralmente denominada de teste de regressão e visa a fornecer evidências de que as mudanças ocorridas não afetam adversamente as características previamente existentes, assim como a evidenciar que as eventuais novas funcionalidades estão de acordo com as expectativas do usuário, ou seja, visa a dar evidências de que o software funciona corretamente após a modificação. A principal diferença entre o teste de regressão e os testes realizados durante o desenvolvimento do software é que durante o teste de regressão pode-se ter de antemão um conjunto de casos de teste disponível para reutilização. Esse conjunto contribui para verificar se novos erros não foram introduzidos com as modificações realizadas.

Recentemente, diversos estudos têm sido realizados abordando o teste de regressão de software. Leung e White (1991) identificam duas abordagens para o teste de regressão: retestartudo (retest-all) e seletiva. $\mathrm{Na}$ abordagem retest-all todos os casos de teste utilizados durante a fase de desenvolvimento são empregados. $\mathrm{Na}$ abordagem seletiva um subconjunto de casos de teste é selecionado a partir do conjunto original identificando partes do programa modificado que devem ser testadas. Ainda, segundo Leung e White, as técnicas de teste de regressão devem ser aplicadas a um baixo custo sem que sua eficácia seja comprometida. Considerando essas duas abordagens, diversas técnicas de teste de regressão têm sido propostas na literatura (Ostrand e Weyuker, 1988; Hartmann e Robson, 1990; Bates e Horwitz, 1993; Chen et al., 1994; Binkley, 1995; Forgács e Takács, 1997; Rosenblum e Weyuker, 1997; Rothermel e Harrold, 1997; Wong et al, 1997a; Wong et al, 1997b; Granja e Jino, 1999). Essas técnicas, e diversas outras existentes 
na literatura, procuram contribuir para a redução do custos e esforços que são aplicados durante a realização das atividades de teste de regressão.

Muitas das técnicas de teste de regressão utilizam mecanismos, técnicas e critérios oriundos da atividade de teste de software realizada durante o processo de desenvolvimento. São exemplos dessas técnicas: Técnica baseada em Fluxo de Dados (Ostrand e Weyuker, 1988) e Técnica baseada em Mutação Seletiva (Wong et al., 1997b). Outros exemplos de técnicas de teste de regressão são: Técnica baseada em Execução Simbólica (Yau e kishimoto, 1987), Técnica baseada em Domínio (Mayrhauser et al., 1994), Técnica baseada em Modificação (Wong et al., 1997a), Técnica baseada em Cobertura (Rothermel e Harrold, 1997), Técnica baseada na Cobertura dos Critérios Potenciais-Uso (Granja, 1997).

A importância de se reduzir o tamanho do conjunto de casos de teste de regressão e de aumentar a eficácia da deteç̧ão de defeitos em programas modificados é ressaltada por Harrold e Wong et al. (1993; 1997a). Em estudos empíricos realizados por Wong et al. (1997a) uma técnica híbrida que combina minimização e seleção baseada em prioridade é proposta. Basicamente, essa técnica identificar um subconjunto representativo de todos os casos de teste que resultam em diferentes comportamentos no programa modificado.

Além da técnica híbrida, Wong et al. (1997b) propõem uma técnica para minimização dos custos da aplicação dos testes de regressão. Basicamente, a técnica utiliza mutação seletiva por meio de um conjunto de operadores de mutação ${ }^{1}$, visando a encontrar um conjunto minimizado de casos de teste a ser aplicado durante os testes de regressão. Nessa técnica, a característica mais importante é examinar como um critério de teste pode ser utilizado no teste de regressão a fim de ajudar os testadores a determinar quais casos de teste devem ser selecionados ou ter uma maior prioridade para o processo de revalidação das novas funcionalidades.

Diante dessa diversidade de técnicas, Rothermel e Harrold (1996) definiram um framework que estabelece características para a comparação e avaliação de técnicas seletivas. Além desse framework, Leung e White (1991) definiram um modelo de custo que compara os custos gastos em aplicar as abordagens de teste de regressão retest-all e seletiva. Tanto o framework quanto o modelo de custo auxiliam testadores a decidir qual abordagem e qual técnica de teste de regressão utilizar. Assim, este trabalho está inserido no contexto de estudos empíricos para contribuir na análise, comparação e escolha de técnicas de teste de regressão utilizando o framework definido por Rothermel e Harrold.

\footnotetext{
' Introduzem pequenos erros sintáticos no programa.
} 


\subsection{Motivação}

O Grupo de Engenharia de Software do Instituto de Ciências Matemáticas e de Computação - ICMC/USP, em colaboração com o Grupo de Engenharia de Software da Faculdade de Engenharia Elétrica da UNICAMP, tem desenvolvido pesquisas na área de teste, com ênfase em estudos teóricos e empíricos e no desenvolvimento de ferramentas de teste. As ferramentas desenvolvidas - POKE-TOOL (Chaim, 1991), PROTEUM (Delamaro, 1993) e PROTEUMIIM (Delamaro, 1997) - possibilitam a realização de trabalhos comparativos entre critérios de teste funcionais, estruturais e baseados em erros. Na linha de teste de regressão, procurando explorar o conhecimento e a experiência do grupo na atividade de teste, iniciou-se uma cooperação com o Dr. Eric Wong tendo sido definida uma estratégia de revalidação com base no critério Análise de Mutantes (Wong et al., 1997b) que motiva o presente trabalho.

Estudos demonstram que mais de $2 / 3$ dos custos durante o ciclo de vida de um software são gastos com a atividade de manutenção e, uma grande porcentagem disto é gasta para a revalidação do software (teste de regressão) (Myers, 1979; Pressman, 1992). Segundo Ostrand (1988), a probabilidade de erros serem introduzirem durante a fase de manutenção está entre 50 e $80 \%$, tornando, assim, a atividade de teste de regressão extremamente importante.

A exemplo do que ocorre na atividade de teste, durante o desenvolvimento de software, várias restrições são impostas à atividade de teste de regressão: custo, tempo, pressões de mercado, e outras. Os custos associados ao teste de regressão são usualmente altos e uma questão pertinente que se coloca é "Como selecionar um subconjunto de casos de teste que diferencie o programa original do programa modificado, ou seja, que revele os possíveis defeitos existentes no programa modificado?", já que à medida que o software evolui, o conjunto de casos de teste aumenta e, conseqüentemente o custo do teste de regressão. Repetir todos os casos de teste é, em geral, impraticável e eliminar arbitrariamente casos de teste é correr riscos. Nessa perspectiva, vários pesquisadores têm investigado técnicas alternativas para selecionar um conjunto de casos de teste de regressão a partir dos casos de teste disponíveis para o programa original (Ostrand e Weyuker, 1988; Bates e Horwitz, 1993; Chen et al., 1994; Binkley, 1995; Rothermel e Harrold, 1997; Wong et al., 1997a; Wong et al, 1997b; Granja e Jino, 1999), e são classificadas segundo Wong et al. (1997a), em duas abordagens: baseadas no comportamento e baseadas na cobertura. 
As técnicas baseadas no comportamento objetivam selecionar todos os casos de teste que apresentam saída diferente para o programa original e o programa modificado, ignorando a cobertura desses casos de teste para o programa modificado. Por outro lado, as técnicas baseadas na cobertura selecionam casos de teste que cobrem componentes modificados e componentes afetados pelas modificações. A diferença de abordagem origina diferentes resultados na seleção dos casos de teste.

\subsection{Objetivos}

Considerando os conceitos apresentados acima, como a importância das atividades de teste de software durante o processo de desenvolvimento de software, a importância das atividades do teste de regressão durante a evolução/manutenção do software e a necessidade de minimização dos custos dessas atividades, este trabalho tem por objetivo realizar estudos empíricos e comparativos utilizando as técnicas de teste de regressão propostas por Wong et al. (1997a, 1997b): Técnica baseada em Modificação e Técnica baseada em Mutação Seletiva.

A Figura 1.1 ilustra as atividades realizadas durante este trabalho e as atividades realizadas por Wong et al. Essas atividades estão caracterizadas na figura por setas azuis tracejadas e por setas vermelhas contínuas, respectivamente.

Procurar-se-á, portanto, complementar e comparar os experimentos realizados por Wong et al. para avaliar ambas as técnicas de teste de regressão, gerando conhecimento e experiência na perspectiva do estabelecimento de estratégias de revalidação eficazes e de baixo custo. Além dos estudos empíricos realizados, uma análise comparativa entre as técnicas de teste de regressão é realizada utilizando o framework desenvolvido por Rothermel e Harrold (1996).

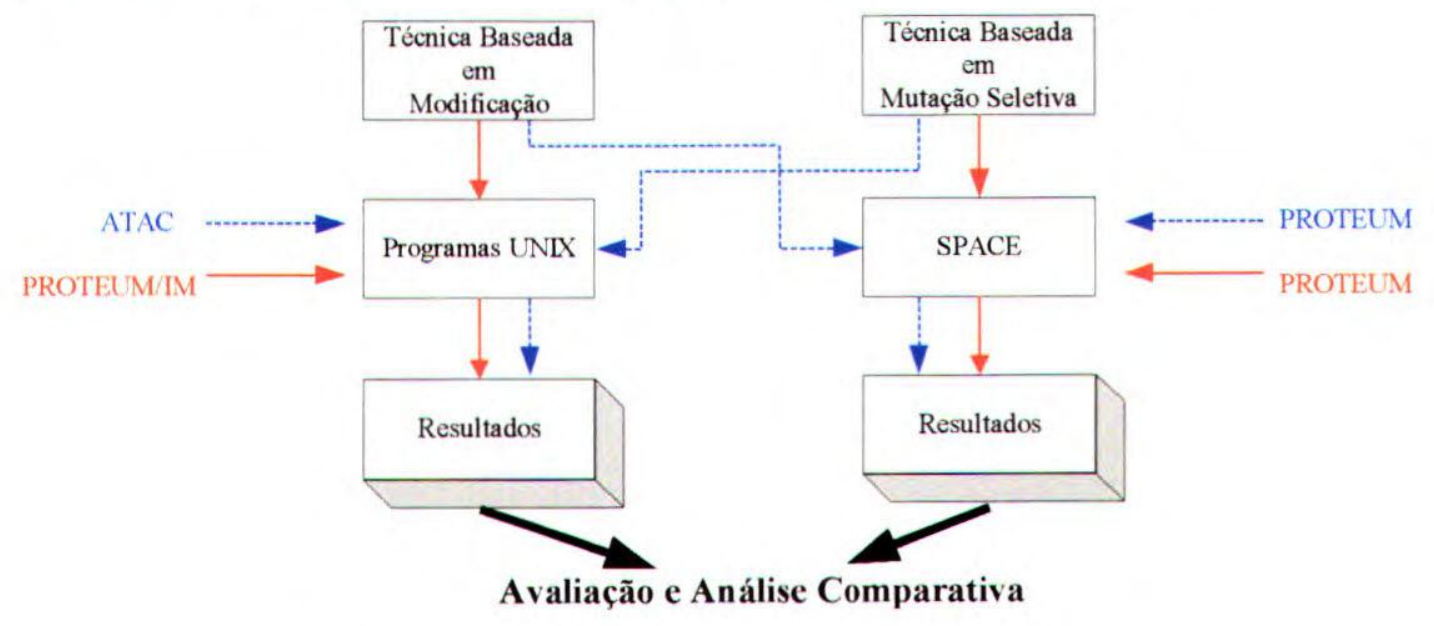

Figura 1.1 - Ilustração das atividades realizadas. 


\subsection{Organização do Trabalho}

Este capítulo apresentou o contexto no qual o trabalho está inserido, a motivação para realizá-lo e os objetivos a serem atingidos. O Capítulo 2 apresenta uma revisão bibliográfica pertinente aos principais conceitos relacionados a este trabalho, enfatizando os aspectos de evolução/manutenção de software e do teste de regressão. No Capítulo 3 são descritos os experimentos realizados com os programas UNIX para avaliar a Técnica baseada em Modificação e os experimentos realizados com o programa SPACE para avaliar a Técnica baseada em Mutação Seletiva. O Capítulo 4 apresenta as contribuições deste trabalho e propostas de trabalhos futuros. O Apêndice A apresenta o conjunto de operadores de mutação utilizado e o Apêndice B apresenta as versões modificadas para os programas UNIX e o processo de ativação dos erros do programa SPACE. 


\section{Capitulo 2}

\section{Revisão Bibliográfica}

\subsection{Considerações Iniciais}

Neste capítulo são apresentados os principais conceitos pertinentes a este trabalho. Primeiramente, são apresentadas a importância da atividade de teste, suas principais técnicas e critérios. Em seguida, são apresentados os principais conceitos relacionados à manutenção de software e à atividade de teste de regressão. As técnicas de teste de regressão pertinentes a este trabalho são descritas, além do framework de avaliação de Rothermel e Harrold (1996) dessas técnicas e o modelo de custo de Leung e White (1991). Ferramentas de teste e estudos empíricos relacionados a este trabalho também são apresentados sucintamente.

\subsection{A Atividade de Teste de Software}

Segundo Myers (1979), o principal objetivo do teste de software é revelar a presença de erros no produto. Idealmente, o programa deveria ser exercitado com todos os valores do domínio de entrada possíveis. Sabe-se, entretanto, que o teste exaustivo é, em geral, impraticável devido às restrições de tempo e custo para realizá-lo.

Myers afirma que:

$>$ Teste é um procedimento de executar um programa com a intenção de encontrar erros.

$>$ Um bom caso de teste é aquele com alta probabilidade de encontrar erros.

$>$ Um teste bem sucedido é aquele que revela um erro ainda não descoberto. 
Assim, o objetivo é determinar quais casos de teste devem ser utilizados de modo que a maioria dos erros existentes possa ser encontrada e que o número de casos de teste utilizado não seja tão grande a ponto de ser impraticável.

Critérios de teste têm sido elaborados com o objetivo de fornecer uma maneira sistemática e rigorosa para selecionar um subconjunto do domínio de entrada e ainda assim ser eficiente para revelar os erros existentes, respeitando as restrições de tempo e custo associados a um projeto de software. Esses critérios são classificados, basicamente, em três técnicas de teste: Técnica Funcional, Técnica Estrutural e a Técnica Baseada em Erros.

\subsubsection{Técnica Funcional}

A técnica funcional, também conhecida como teste de caixa preta, trata o software como uma caixa cujo conteúdo é desconhecido, sendo possível visualizarem-se somente os dados de entrada e as respostas produzidas como saída. Nessa técnica são verificadas as funções do sistema sem se preocupar com detalhes de implementação. Para isto, Coward (1988) distingue nessa técnica dois passos principais: primeiro, identificar as funções esperadas do software e segundo, criar casos de teste que chequem a corretitude dessas funções.

Um componente importante do teste funcional é o oxáculo. O oráculo determina se a saída obtida, após a execução de uma função com os casos de teste, é correta ou não. A partir de ferramentas que simulem a especificação do sistema é possível obterem-se as informações necessárias para o oráculo; entretanto, na maioria das vezes, o oráculo é a figura do próprio testador (Howden, 1985; Biezer, 1990).

Os critérios de teste funcional mais conhecidos são: Particionamento em Classes de Equivalência, Análise do Valor Limite, Grafos de Causa e Efeito e Error Guessing.

Particionamento em classes de equivalência divide o domínio de entrada de um programa em classes, a partir das quais os casos de teste podem ser derivados. 0 objetivo é minimizar o número de casos de teste, obtendo-se somente casos de teste essenciais, ou seja, que possuam alta probabilidade de revelar a presença dos erros existentes no programa. $O$ uso de particionamento permite examinar os requisitos com mais detalhes e restringir o número de casos de teste existentes.

Análise do valor limite procura testar os limites das condições de entrada. Segundo Pressman (1992), erros costumam ocorrer com mais freqüência nos limites dos domínios de entrada do que no centro desses domínios, tornando o critério Análise de valor limite relevante para o teste funcional de um sistema. 
Grafo de causa e efeito é utilizado para testar o efeito combinado de dados de entrada. Causas e efeitos são identificados e combinados em um grafo a partir do qual uma tabela de decisão é criada. Os dados de teste e as saídas são derivados da tabela de decisão obtida.

Error guessing consiste basicamente em listar possíveis erros e construir casos de teste associados a essas condições de erros.

Em geral, o teste funcional é uma técnica de validação de programas na qual os casos de teste são gerados a partir da especificação dos requisitos tornando-se, assim, uma técnica sujeita às inconsistências que podem ocorrer na especificação (DeMillo, 1987).

\subsubsection{Técnica Estrutural}

Essa técnica, também conhecida como teste de caixa branca, estabelece os requisitos de teste baseando-se nos aspectos de implementação, ou seja, no conhecimento da estrutura interna do programa. A maioria dos critérios dessa técnica utiliza uma representação de programa conhecida como grafo de fluxo de controle (GFC) ou grafo de programa. Um GFC é um grafo orientado no qual cada vértice representa um bloco indivisível de comandos e cada aresta representa um desvio de um bloco para outro. Um bloco desse tipo tem as seguintes características: não existem desvios para o meio do bloco e uma vez que o primeiro comando do bloco seja executado, todos os demais comandos do bloco são executados seqüencialmente. Utilizando-se do grafo de programa, os componentes que devem ser executados são escolhidos, caracterizando assim o teste estrutural.

Os critérios estruturais baseiam-se em tipos de estruturas diferentes para determinar quais partes do programa são requeridas na execução. Como critérios de teste estrutural tem-se: Critérios Baseados em Fluxo de Controle, Baseados em Fluxo de Dados e Baseados na Complexidade.

Os critérios baseados em fluxos de controle utilizam-se de características de controle da execução do programa, como comandos ou desvios, para determinar quais estruturas são necessárias. Os mais conhecidos são os critérios Todos-Arcos, Todos-Nós e Todos-Caminhos. Os critérios Todos-Nós e Todos-Arcos exigem que cada comando e cada aresta (cada desvio do programa) do GFC sejam exercitados pelo menos uma vez. Já o critério Todos-Caminhos que, geralmente, é impraticável, requer que todos os caminhos possíveis do programa sejam executados (Pressman, 1992). Um caminho (completo) é executável se existe um conjunto de valores que possa ser atribuído às variáveis de entrada do programa e que causa a execução desse caminho, caso contrário, esse caminho é dito não executável (Frankl, 1987). 
Os critérios baseados em fluxo de dados utilizam-se de informações do fluxo de dados do programa para estabelecer os requisitos de teste. Esses critérios requerem que sejam testadas as interações que envolvam definições de variáveis e referências a essas definições. Exemplos dessa classe de critérios são os Critérios de Rapps \& Weyuker (Rapps e Weyuker, 1982; Rapps e Weyuker, 1985) e os Critérios Potenciais-Usos (Maldonado, 1991).

Os critérios baseados na complexidade utilizam-se de informações sobre a complexidade do programa para determinar os requisitos de teste. Um critério bastante conhecido dessa classe é o critério de McCabe que utiliza a complexidade ciclomática ${ }^{2}$ para estabelecer os requisitos de teste. Essencialmente, esse critério requer que um conjunto de caminhos linearmente independente do grafo de programa seja executado (Pressman, 1992).

\subsubsection{Técnica Baseada em Erros}

Essa técnica enfatiza os erros que o programador ou o projetista pode cometer durante o processo de desenvolvimento $e$ as abordagens que podem ser usadas para revelar a sua ocorrência. A Análise de Mutantes e a Mutação de Interface (Interface Mutation) são critérios típicos dessa técnica.

A Análise de Mutantes e a Mutação de Interface são critérios de teste que, para avaliar o quanto um conjunto de casos de teste $\mathbf{T}$ é adequado para o teste de um dado programa $\mathbf{P}$, utilizam um conjunto de programas, ligeiramente diferentes de $\mathbf{P}$, chamados de mutantes. $\mathbf{O}$ objetivo é obter casos de teste que consigam revelar as diferenças de comportamento existentes entre $\mathbf{P}$ e seus mutantes (DeMillo, 1980).

O critério Análise de Mutantes será descrito com mais detalhes por ser base para este trabalho. A sintese apresentada foi retirada do trabalho de Souza (1996).

\subsubsection{O Critério Análise de Mutantes}

A Análise de Mutantes surgiu na década de 70 na Yale University e Georgia Institute of Technology (DeMillo, 1978) e vários trabalhos empíricos e teóricos têm indicado que esse critério é atrativo para o teste de programas (Budd e DeMillo, 1980a; Mathur, 1991; Mathur e Wong, 1993; Wong, 1993; Mathur e Wong, 1994; Wong e Mathur, 1995a; Wong e Mathur, 1995b; Offut et al., 1996a; Offut et al., 1996b). Basicamente, a idéia da Análise de Mutantes é

\footnotetext{
${ }^{2}$ Medida de software que permite medir quantitativamente a complexidade lógica de um programa. Possibilita estabelecer um limite superior para o número de testes que devem ser conduzidos para garantir que todas as declaraçōes de um programa sejam executadas pelo menos uma vez.
} 
criar a confiança de que um programa $\mathrm{P}$ está correto produzindo-se, por meio de pequenas alterações sintáticas, um conjunto de programas, chamados de mutantes, semelhantes a $\mathbf{P}$, e construindo-se casos de teste capazes de provocar diferenças de comportamento entre $\mathbf{P}$ e seus mutantes. Essas alterações são feitas utilizando-se de um conjunto de operadores denominados operadores de mutação. A cada operador associa-se um tipo ou uma classe de erros que se pretende revelar.

Segundo DeMillo (1987), a Análise de Mutantes é um critério para medir a adequação dos casos de teste, no qual um conjunto de casos de teste é dito adequado se o programa funciona corretamente quando executado com os casos de teste e se todos os programas incorretos têm um comportamento não esperado com alguns desses casos de teste. Dessa forma, o objetivo inicial da Análise de Mutantes era de servir como um meio de medir o quanto um conjunto de casos de teste é adequado a um determinado programa, sendo demonstrado por DeMillo (1978) sua eficácia também para revelar erros em programas.

A Análise de Mutantes consiste de quatro etapas principais: geração de mutantes, execução de $\mathbf{P}$ com base em um dado conjunto de casos de teste $\mathbf{T}$, execução dos mutantes com base em $\mathbf{T}$ e análise dos mutantes. $\mathbf{O}$ principal objetivo é encontrar um conjunto de casos de teste que mate todos os mutantes não equivalentes a $\mathbf{P}$. Tais conjuntos são considerados adequados para o teste de $\mathbf{P}$, no sentido de que ou $\mathbf{P}$ está correto ou contém um erro sutil e inesperado.

Durante a Análise de Mutantes um programa $\mathbf{P}$ é testado com um conjunto de casos de teste T. Se o programa funciona corretamente, então este sofre pequenas modificações, gerando seus mutantes que são executados com $\mathbf{T}$. Caso o comportamento de um mutante de $\mathbf{P}$ seja diferente de $\mathbf{P}$, então esse mutante é dito "morto". Caso contrário, o mutante permanece vivo devido a um dos dois motivos a seguir: 1. o conjunto $\mathbf{T}$ não é suficiente (adequado) para distinguir o comportamento de $\mathbf{P}$ e seu mutante e, com isso, novos casos de teste devem ser incluídos ao conjunto ou; 2. o mutante é dito equivalente a $\mathbf{P}$, ou seja, para qualquer dado do domínio de entrada o comportamento de ambos os programas não diferem.

Uma das dificuldades para a aplicação desse critério é a determinação de equivalência, em geral indecidivel (Budd, 1981). Usualmente requer a intervenção do testador ou a elaboração de heurísticas para decidir sobre encerrar a atividade de teste com base nesse critério. Dois pontos relevantes são o projeto e a implementação de um conjunto de operadores de mutação que, essencialmente, modelam os tipos de erros que se deseja revelar. Tanto o projeto quanto à implementação baseiam-se na hipótese do programador competente que afirma que um programador competente produz programas corretos ou perto do correto. Além disso, cada 
mutante é gerado pela aplicação de um único operador, considerando o efeito de acoplamento. $O$ efeito de acoplamento afirma que casos de teste que são capazes de revelar erros simples também sāo capazes de revelar erros mais complexos (DeMillo, 1978).

Conseguindo-se obter casos de teste que resultem em apenas mutantes mortos e equivalentes tem-se um conjunto de casos de teste adequado ao programa em teste.

Um escore de mutação é definido para verificar a adequação do conjunto de casos de teste utilizado. $O$ escore de mutação é calculado a partir da seguinte equação:

$$
m s(P, T)=\frac{D M(P . T)}{M(P)-E M(P)}
$$

na qual:

$\mathrm{DM}(\mathrm{P}, \mathrm{T})$ : Número de mutantes mortos pelo conjunto de casos de teste $\mathbf{T}$.

$M(P)$ : Número de mutantes gerados para o programa $\mathbf{P}$.

EM(P): Número de mutantes equivalentes ao programa $\mathbf{P}$.

O escore de mutação varia no intervalo de [0,1]; quanto maior o escore mais adequado é o conjunto de casos de teste para o programa emxteste. Percebe-se com essa fórmula que somente $\mathbf{D M}(\mathbf{P}, \mathbf{T})$ é dependente do conjunto de casos de teste utilizado e que EM(P) é obtido à medida que o testador decide que um determinado mutante vivo é equivalente, o que pode ser feito interativamente ou automaticamente aplicando-se heurísticas.

A Análise de Mutantes fornece uma medida objetiva do nível de confiança na adequação dos casos de teste analisados. Com o escore de mutação, que relaciona o número de mutantes gerados com o número de mutantes mortos, pode-se avaliar a adequação dos casos de teste usados e, como conseqüência, a confiabilidade do programa testado.

Um outro problema para a aplicação do critério, e que por algum tempo foi um limitante para o seu uso, é o seu custo em termos de tempo de execução, pois o número de mutantes criados, mesmo para programas pequenos, pode ser muito grande, demandando um tempo de execução demasiadamente alto. Com os recursos computacionais disponiveis atualmente e com algumas estratégias torna-se possível empregar a Análise de Mutantes dentro de limites aceitáveis de tempo (Souza, 1996).

Entre as pesquisas relacionadas com os aspectos de custo de aplicação do critério Análise de Mutantes, têm sido investigadas abordagens para a redução do número de mutantes sem a fetar 
a eficácia do critério (Mathur e Wong, 1993; Mathur e Wong, 1994; Offut et al.,1996a; Offut et $a l .$, 1996b). Uma linha interessante é a caracterização de um subconjunto de operadores de mutação que apresente o mesmo grau de adequação e eficácia, mas a um custo menor. Os critérios que abordam essa caracterização têm sido denominados de Mutação Restrita (Constrained Mutation) (Mathur, 1991; Wong, 1993), Mutação Aleatória (Acree et al., 1979) e Mutação Seletiva (Offut et al., 1993; Barbosa, 1998).

A Mutação Restrita seleciona alguns operadores específicos para a geração de mutantes. A partir desse conjunto, é possível obter uma sensível redução no custo de execução dos mutantes sem reduzir a capacidade do critério em revelar erros. A Mutação Aleatória, a invés de considerar todos os mutantes gerados, seleciona aleatoriamente uma porcentagem de mutantes e a análise fica restrita a esses mutantes selecionados. A Mutação Seletiva propõe que os operadores de mutação responsáveis por gerar um maior número de mutantes não sejam aplicados.

O critério Análise de Mutantes para o teste de programas em $\mathrm{C}$ é apoiado pela ferramenta de teste PROTEUM desenvolvida por Delamaro (1993) e descrita na Seção 2.6.1.

\subsubsection{O Critério Mutação de Interface}

Esse critério de teste é responsável por revelar erros de integração. Possui o mesmo princípio do critério Análise de Mutantes criando mutantes inserindo pequenas perturbações nas conexões entre módulos de um programa.

Utilizando o mesmo raciocínio aplicado à Análise de Mutantes, casos de teste capazes de distinguir mutantes de interface (interface-mutants) também devem ser capazes de revelar grande parte dos erros de integração (Vincenzi, 1998). Essa informação depende de quais mutantes são utilizados, ou seja, quais operadores de mutação são aplicados (Delamaro, 1997b).

O critério Mutação de Interface explora três idéias básicas:

$>$ Restringir os operadores de mutação a fim de modelar apenas erros de integração.

$>$ Testar as conexões entre módulos separadamente, uma de cada vez.

$>$ Aplicar os operadores de mutação somente nas partes relacionadas às interfaces dos módulos, tais como chamadas de função, parâmetros ou variáveis.

O critério Mutação de Interface para o teste de integração de programas escritos em C é apoiado pela ferramenta de teste PROTEUM/IM desenvolvida por Delamaro (1997) e descrita na Seção 2.6.1. 


\subsection{A Atividade de Teste de Regressão}

Estudos mostram que cerca de $2 / 3$ do esforço total do ciclo de vida de um sistema de software é gasto durante as atividades de manutenção (Myers, 1979; Ostrand e Weyuker, 1988; Pressman, 1992; Rothermel e Harrold, 1996). Assim, o teste de software realizado após alguma modificação é tão importante quanto o teste realizado durante o processo de desenvolvimento do software.

Um software sofre modificações por várias razões, mudanças de especificação, adaptação a um novo ambiente, etc. Existem, basicamente, 4 tipos de modificações realizadas durante a manutenção (Hartmann e Robson, 1990):

Adaptativa: modificações são realizadas para que o software se adapte a um novo ambiente.

Corretiva: modificações são realizadas para corrigir erros.

$>$ Preventiva: modificações são realizadas para facilitar futuras manutenções.

$>$ Evolutiva: modificações são realizadas para adicionar novas funcionalidades.

Qualquer modificação, independentemente do tipo, pode efetivamente alterar a estrutura do programa, portanto, deve ser testada. Modificações corretivas e evolutivas requerem novos casos de teste funcionais, enquanto que, modificações adaptativas e preventivas não requerem necessariamente novos casos de teste, pois, eventualmente, não implicam mudanças nos requisitos.

As áreas modificadas devem ser tão revalidadas quanto às áreas que não sofreram modificações com o propósito de garantir que funcionalidades não modificadas não tenham sido afetadas (White e Leung, 1992). Conforme caracterizado anteriormente, o processo de teste realizado durante a fase de manutenção/evolução do software é denominada teste de regressão. Portanto, teste de regressão é uma parte do processo de manutenção que utiliza casos de teste previamente desenvolvidos para revelar erros nas funcionalidades não modificadas e nas novas funcionalidades, devendo ser aplicado tanto ao nível de unidade quanto aos níveis de sistema $\mathrm{e}$ integração. Os erros eventualmente encontrados durante a fase de manutenção são chamados de erros de regressão (Abdullah et al., 1995).

Idealmente, quando se testa um programa que sofreu modificações, todos os casos de teste existentes devem ser reutilizados a fim de determinar se modificaçōes introduziram 
defeitos. Utilizar um conjunto de casos de teste já planejado minimiza os esforços para criar novos casos de teste e também permite uma comparação direta com a saída do programa modificado com a saída do programa original. No entanto, testar todos os conjuntos de casos de teste existentes é muito custoso. Assim, um subconjunto de casos de teste é selecionado para retestar o programa modificado. Às vezes, os casos de teste existentes não são suficientes para avaliar as modificações no software, assim, novos casos de teste devem ser adicionados.

Uma característica importante do teste de regressão é que durante os testes pode-se ter de antemão um conjunto de casos de teste disponível para reutilização. Esse conjunto permite verificar se novos erros não foram introduzidos com as modificaçōes realizadas.

Outro ponto importante a ser ressaltado é o fato de que o teste de regressão é distinguido em duas fases (Rothermel e Harrold, 1996):

Fase Preliminar: inicia-se logo após a nova versão ser finalizada; durante essa fase os desenvolvedores corrigem o software preparando-o para a nova versão. Enquanto isso, os testadores planejam as atividades de testes ou realizam tarefas de coletar informações de trace e análise de cobertura. Quando as correções estão concluídas, inicia-se a próxima fase.

$>$ Fase Crítica: durante essa fase o teste de regressão é realizado. É nessa fase que os custos são maiores.

Apesar das vantagens do teste de regressão, existem basicamente dois problemas principais, os quais as técnicas existentes procuram solucionar. $\mathrm{O}$ primeiro - test-update problem - é a preocupação em manter o conjunto de casos de teste $\mathbf{T}$ ainda adequado após as modificações, pois identificar casos de teste irrelevantes e eliminá-los não é tarefa fácil. $O$ segundo - test-selection problem - é a preocupação em selecionar quais casos de teste devem ser utilizados para retestar o programa após modificações. É importante que os casos de teste sejam selecionados sistematicamente, pois executar um conjunto inteiro de casos de teste adequados para validar poucas modificações pode consumir muito tempo e recursos computacionais e humanos (Hartmann e Robson, 1990). 
Em geral, a atividade de teste de regressão segue os seguintes passos:

1. Identificar as modificações realizadas no programa.

2. Selecionar o conjunto de casos de teste, a partir do conjunto original $T$, a ser reexecutado no teste de regressão.

3. Aplicar o conjunto selecionado ao programa modifica do e avaliar os resultados.

4. Gerar novos casos de teste, se necessário.

5. Aplicar os novos casos de teste e avaliar os resultados.

6. Estabelecer a base de dados dos casos de teste utilizado no teste de regressão.

Diversas técnicas são propostas na literatura para auxiliar sistematicamente o teste de regressão. Algumas dessas técnicas são descritas sucintamente a seguir.

A Técnica Baseada em Fluxo de Dados propõe o uso da análise de fluxo de dados para selecionar e avaliar casos de teste para serem utilizados durante o teste de regressão [OST88]. A análise de fluxo de dados pode ser utilizada para selecionar casos de teste de regressão a partir de um conjunto de casos de teste já definido, e gerar casos de teste adicionais. A análise de fluxo de dados permite selecionar casos de teste para todas essas situaçōes. A teoria do teste de regressão utilizando a análise de fluxo de dados é executar todos os caminhos que sofreram modificações. Na mesma linha de pesquisa baseada em fluxo de dados, a Técnica baseada na Cobertura dos Critérios Potenciais-Uso, proposta por Granja (1997), seleciona os casos de teste para serem utilizados no teste de regressão a partir dos elementos requeridos para os critérios Potenciais-Uso que foram modificados.

A Técnica Baseada em Execução Simbólica, proposta por Yau e Kishimoto (1987), divide o domínio de entrada em diferentes classes utilizando o código e a especificação do programa modificado e seleciona um caso de teste de cada classe para executar o programa modificado. $\mathrm{O}$ objetivo é executar cada modificação ou cada novo código pelo menos um vez. $\mathrm{O}$ particionamento do domínio é baseado na especificação a fim de obter diferentes combinações dos dados de entrada e a identificação de possíveis caminhos através do código, estabelecendo que cada classe de entrada deve ser testada pelo menos uma vez. Assim, casos de teste são alocados a diferentes partições de entrada e executados utilizando execução simbólica.

A Técnica Baseada em Domínio, proposta por Mayrhauser et al. (1994), gera casos de teste baseando-se na análise e no modelo do domínio. Os modelos de domínio são utilizados 
como uma estrutura para gerar casos de teste e armazenam informações sintáticas e semânticas necessárias para a geração de novos casos de teste.

A Técnica baseada em Cobertura, proposta por Rothermel e Harrold (1997), seleciona casos de teste de regressão percorrendo os grafos de fluxo de controle dos programas originais e modificados. Basicamente, o algoritmo constrói o grafo de fluxo de controle para os programas $P$ e $\mathrm{P}^{\prime}$, coleta traces de teste que associam casos de teste em $\mathrm{T}$ com arcos dos grafos. Durante o percurso, o algoritmo compara as declarações associadas com os nós que são simultaneamente alcançados em ambos os grafos. Quando o algoritmo encontra um par de nós em $\mathrm{N}$ e $\mathrm{N}^{\prime}$ dos grafos de $\mathrm{P}$ e $\mathrm{P}^{\prime}$, respectivamente, tal que as declarações associadas a $\mathrm{N}$ e $\mathrm{N}^{\prime}$ não sejam lexicamente idênticas, o algoritmo seleciona todos os casos de teste em $\mathrm{T}$ que alcançam $\mathrm{N}$ em $\mathrm{P}$.

Diante da diversidade de técnicas de teste de regressão existentes, são necessários mecanismos que possibilitem avaliar e comparar essas técnicas. Rothermel e Harrold (1996) propuseram um framework para avaliar e comparar técnicas baseadas na abordagem seletiva. Além disso, Leung e White (1991) propõem um modelo de custo para comparar as técnicas baseadas na abordagem seletiva e as técnicas baseadas na abordagem retest-all. Nas seções 2.3.1 e 2.3.2 esses mecanismos são apresentados, respectivamente.

Nas seções 2.4 e 2.5 são apresentadas com mais detalhes as técnicas que foram utilizadas no escopo deste trabalho: Técnica baseada em Modificacão e Técnica baseada em Mutação Seletiva.

\subsubsection{Framework de Avaliação e Comparação de Técnicas de Teste de Regressão Seletivas}

Algumas técnicas de teste de regressão baseiam-se na especificação do software para selecionar os testes de regressão, enquanto outras se baseiam no código original (P) e no código modificado (P'). Essas últimas técnicas têm os seguintes objetivos (Rothermel e Harrold, 1996; Souza, 1997):

Cobertura - Selecionar casos de teste que passem pelos componentes modificados.

Minimização - Semelhante à anterior: selecionar um conjunto mínimo de casos de teste de regressão que passem pelos componentes modificados.

$>$ Segurança - Selecionar casos de teste de T que revelam defeitos em $\mathbf{P}$ '. 
Como são muitas as técnicas seletivas de teste de regressão, é preciso uma maneira de compará-las e avaliá-las. Com esse objetivo, Rothermel e Harrold (1996) propuseram um framework que define algumas características que auxiliam na comparação e avaliação das técnicas: inclusão (inclusiveness), precisão, eficiência, e generalidade.

Inclusão - Mede a extensão com a qual a técnica inclui casos de teste que fazem com que P' produza uma saída diferente de $\mathbf{P}$ e revela os defeitos.

$>$ Precisão - Mede a habilidade da técnica em evitar testes que não fazem com que $\mathbf{P}^{\prime}$ produza um saída diferente de $\mathbf{P}$.

$>$ Eficiência - Mede o custo computacional gasto pela técnica.

$>$ Generalidade - Mede a habilidade da técnica em manipular linguagens diversas.

Segundo Rothermel e Harrold, uma técnica seletiva de teste típica segue o seguinte:

1. Selecionar um conjunto de casos de teste $\mathbf{T}^{\prime} \subseteq \mathbf{T}$ para executar $\mathbf{P}$.

2. Testar P' com 'T' para estabelecer a corretitude de $\mathbf{P}$ ' com respeito a T'.

3. Se necessário, criar $\mathbf{T}$ ", um conjunto de novos casos de teste para $\mathbf{P}$ '.

4. Testar P' com T' para estabelecer a corretitude de $\mathbf{P}$ ' com respeito a $T^{\prime \prime}$.

5. Criar T"', um novo teste adequado para P', a partir de T, T' e T".

Para a definição do framework, os autores dividem os casos de teste em três classes: fault-revealing, modification-revealing e modification-traversing.

Fault-revealing são os casos de teste capazes de revelar os erros existentes no programa. Entretanto, não existe um mecanismo eficiente para encontrar esses casos de teste a partir de um conjunto inicial. Assim, tem-se a seguinte indagação que dificilmente é possível responder: Como saber se um caso de teste revela erros antes de aplicá-lo?

Modification-revealing são os casos de teste que revelam um comportamento diferente entre P e P'. Entretanto, para selecioná-los seria necessário executar todo o conjunto de casos de teste para saber quais são modification-revealing. Então, a solução é selecionar aqueles casos de teste que passam pelas modificações feitas no programa: os casos de teste modificationtraversing, que incluem os casos de teste fault-revealing e modification-revealing. Assim, os casos de teste modification-traversing são aqueles com maiores chances de revelarem defeitos. A Figura 2.1 mostra a relação entre as três diferentes classes de conjuntos de casos de teste. 
Além das três classes de conjuntos de casos de teste definidos para este framework, têmse os casos de teste obsoletos e não obsoletos. Os casos de teste obsoletos são aqueles que não serão reutilizados para as atividades de teste de regressão, enquanto que os casos de teste não obsoletos são aqueles que serão reutilizados. Ambos os casos de teste estão caracterizados na Figura 2.1.

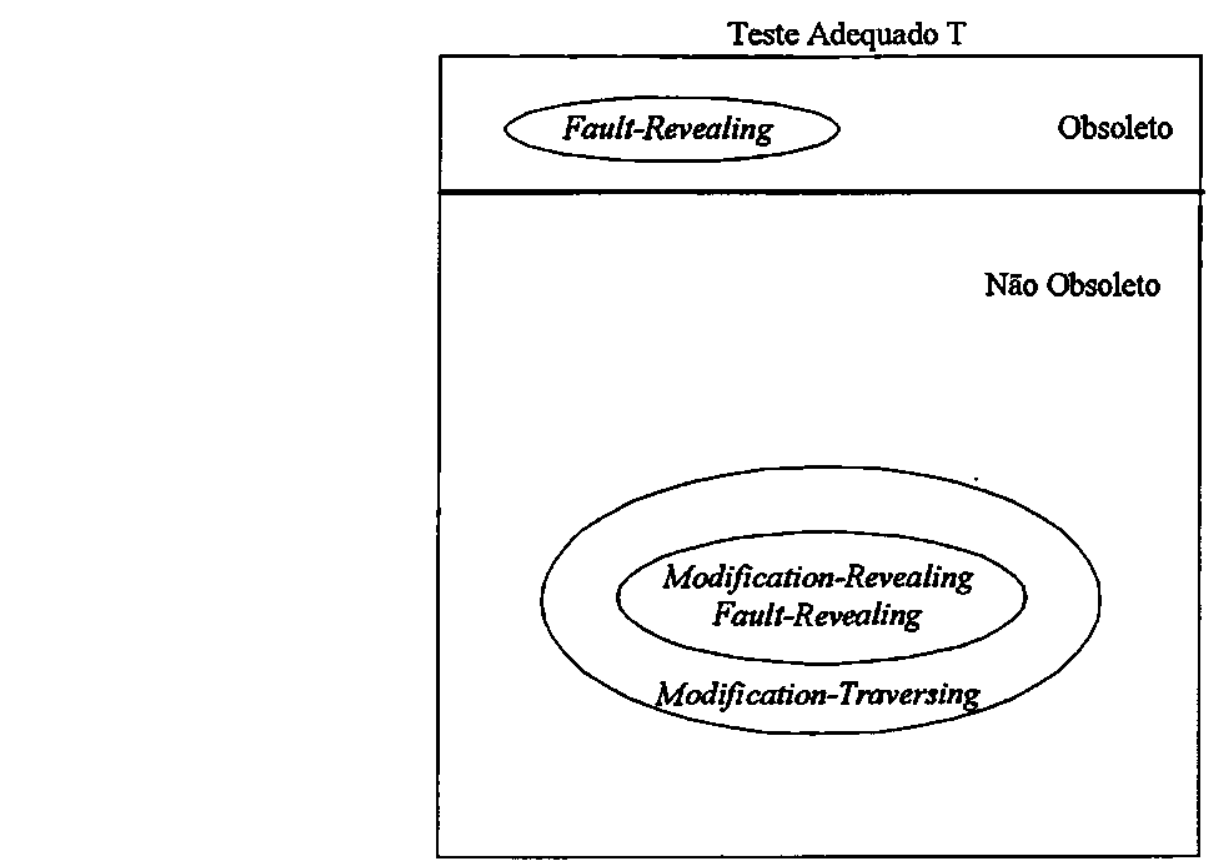

Figura 2.1 - Relacionamento entre as três classes de conjuntos de casos de teste (Rothermel e Harrold, 1996).

As três classes de casos de teste descritas podem contribuir para a especificação do framework para avaliar e comparar técnicas de teste de regressão baseadas na estratégia seletiva por várias razões:

Testadores são relutantes quanto ao fato de descartar casos de teste que possam revelar erros. $\mathrm{O}$ relacionamento entre as três classes de casos de teste fornece uma maneira de avaliar analiticamente as técnicas de teste baseadas na abordagem seletiva em termos de suas habilidades de selecionar casos de teste que sejam fault-revealing e casos de teste que não o sejam. Apesar do fato de que essas técnicas objetivam selecionar casos de teste para satisfazer alguma medida de adequação, é razoável e importante avaliar essas técnicas em termos de suas habilidades de revelar defeitos.

$>$ As três classes podem ainda servir para distinguir técnicas de teste de regressão baseadas na abordagem seletiva. Muitas técnicas são mais precisas, eliminando do conjunto de 
casos de teste aqueles não que executam componentes modificados e aqueles que não causam saídas diferentes em $\mathbf{P}$ e $\mathbf{P}^{\prime}$. Assim, é útil comparar técnicas de teste de regressão em termos de suas habilidades em identificar tais classes de casos de teste.

A seguir, cada uma das quatro caracteristicas que compõem o framework é apresentada. A seguinte notação é utilizada: $\mathbf{M}=$ técnica; $\mathbf{P}=$ programa original; $\mathbf{P}$ ' = programa modificado; $\mathbf{T}=$ conjunto de casos de teste original; e $\mathbf{T}^{\boldsymbol{\prime}}=$ subconjunto de casos de teste de regressão, $\mathbf{T}^{\mathbf{9}} \subseteq$ T.

\section{Inclusão}

Mede a extensão com a qual a técnica $\mathbf{M}$ seleciona casos de teste modification-revealing a partir de $\mathbf{T}$ para incluir em $\mathbf{T}^{\prime}$. Assim, tem-se:

Definição 1 - Supondo que T contenha n casos de teste que sejam modification-revealing para $\mathbf{P}$ e $\mathbf{P}^{\prime}$, e supondo que $\mathbf{M}$ selecione $\mathbf{m}$ desses casos de teste, a inclusão de $\mathbf{M}$ relativa a $\mathbf{P}, \mathbf{P}^{\prime}$ e T é:

(1) a porcentagem dada pela expressão $(100(m / n))$ se $n \neq 0$ ou

(2) $100 \%$ se $n=0$.

Por exemplo, se $\mathbf{T}$ contém 50 casos de teste dos quais 8 são modification-revealing para $\mathbf{P}$ e $\mathbf{P}^{\prime}$, e $\mathbf{M}$ seleciona 2 destes 8 , então a inclusão de $\mathbf{M}$ relativa a $\mathbf{P}, \mathbf{P}^{\prime}$ e $\mathbf{T}$ é $25 \%$.

Definição 2 - Se para todo $\mathbf{P}, \mathbf{P}^{\prime}$ e T, a inclusão de $\mathbf{M}$ relativa a $\mathbf{P}, \mathbf{P}^{\prime}$ e $\mathbf{T}$ é $100 \%$, então M é segura.

Inclusão e segurança são medidas importantes e bastante significantes. Se uma técnica de teste de regressão M1 tem maior inclusão que uma técnica M2, então M1 tem maior habilidade em revelar defeitos do que $\mathbf{M 2}$.

\section{Precisão}

Mede a extensão com a qual $\mathbf{M}$ omite casos de teste que não são modification-revealing. Assim, tem-se: 
Definição 3 - Supondo que $\mathbf{T}$ contenha $\mathbf{n}$ casos de teste que não são modificationrevealing para $\mathbf{P}, \mathbf{P}^{\prime}$ e $\mathbf{T}$ e supondo que $\mathbf{M}$ omite $\mathbf{m}$ destes casos de teste. $\mathbf{A}$ precisão de $\mathbf{M}$ relativa a $\mathbf{P}, \mathbf{P}$ e $\mathbf{T}$ é:

(1) a porcentagem dada pela expressão $(100(m / n))$ se $n \neq 0$ ou

(2) $100 \%$ se $n=0$.

Por exemplo, se $\mathbf{T}$ contém 50 casos de teste dos quais 44 não são modification-revealing para $\mathbf{P}, \mathbf{P}^{\prime}$ e $\mathbf{T}$, e $\mathbf{M}$ omita 33 desses 44 , então a precisão de $\mathbf{M}$ relativa a $\mathbf{P}, \mathbf{P}^{\prime}$ e $\mathbf{T}$ é $75 \%$.

A precisão é bastante útil, pois mede a extensão com a qual $\mathbf{M}$ evita selecionar testes que não causam um comportamento diferente no programa modificado. Em geral, quando se compara técnicas de teste em termos de precisão, é possível identificar as técnicas que menos escolhem casos de teste desnecessários. Quando se compara segurança em termos de precisão, pode-se identificar técnicas que se aproximam do objetivo principal que é selecionar casos de teste que sejam modification-revealing.

\section{Eficiência}

A eficiência de uma técnica é medida em termos do espaço utilizado para armazenar os dados de teste e em termos do tempo gasto para aplicar e avaliar a técnica. Quando tempo é considerado, a estratégia seletiva é mais econômica que a estratégia retest-all se o custo de selecionar $\mathbf{T}^{\boldsymbol{N}}$ é menor que o custo de executar os casos de teste em $\mathbf{T}$ (Leung e White, 1991).

Pode-se identificar fatores importantes que influenciam na avaliação da eficiência de uma técnica:

$>$ Fases que são realizadas durante o teste de regressão: fase preliminar e fase crítica.

$>$ Esforço humano empregado durante o teste de regressão. Técnicas que requerem muito esforço humano são impraticáveis.

Extensão com a qual a técnica deve calcular as informações nos módulos modificados. Uma técnica que deve determinar cada componente removido, modificado ou adicionado a $\mathbf{P}$ ou calcular semelhanças entre $\mathbf{P}$ e $\mathbf{P}^{\prime}$ pode ser mais cara que aquela técnica que verifica essas modificações apenas quando necessário.

Habilidade da técnica em manipular casos nos quais P' é criado a partir de várias modificações de $\mathbf{P}$. Uma técnica que depende da análise de programas e processa uma 
modificação de cada vez pode ser forçada a reavaliar todas as informações obtidas após cada modificação. Tal análise pode ser cara e pode aumentar o custo de aplicação da técnica.

\section{Generalidade}

A generalidade de uma técnica seletiva é a habilidade da técnica em funcionar em diversas situações. Fatores que devem ser considerados ao avaliar a generalidade de uma técnica:

Para ser prática, a técnica deve funcionar para vários tipos de programas. Por exemplo, uma técnica que é definida somente para procedimentos com if, while, e atribuições não é prática.

$>$ Uma técnica deve manipular modificações realistas. Por exemplo, uma técnica que não manipula modificações que alteram o fluxo de controle, em geral, não é prática.

$>$ Uma técnica que depende do ambiente de teste e de manutenção é menos geral que uma que não depende destes fatores.

$>$ Uma técnica que depende da disponibilidade de ferramentas de análise é menos geral que uma técnica que não depende de tais ferramentas. Por exemplo, uma técnica que requer informações sobre trace de casos de teste é menos geral que uma técnica que não requer essas informações.

$>$ Uma técnica pode apoiar teste intraprocedural ou interprocedural. Na prática, teste de regressão é freqüentemente realizado em nível interprocedural.

Pode-se definir generalidade mais quantitativamente. No entanto, neste framework, as comparações qualitativas são suficientes, segundo Rothermel e Harrold (1996).

Com o objetivo de utilizar e avaliar o framework definido, Rothermel e Harrold conduziram um experimento aplicando várias técnicas de teste de regressão seletivas em fragmentos de programas modificados. Cada uma das técnicas foi aplicada e avaliada de acordo com as características definidas no framework. A partir desse experimento, Rothermel e Harrold estabeleceram os seguintes pontos:

Aumentar a precisão pode diminuir a eficiência em técnicas seguras.

$>$ Aumentar a precisão ou a inclusão pode diminuir a eficiência em técnicas não seguras. 
$>$ A maioria dos fatores que afeta a generalidade também pode afetar a inclusão, a precisão e a eficiência.

$>$ Uma técnica que deve reavaliar todas as informaçōes já obtidas a cada modificação pode influenciar a eficiência da técnica. Por outro lado, se a reavaliação não ocorre a precisão da técnica pode ser influenciada.

\subsubsection{Um Modelo de Custo para Comparar Técnicas Seletivas e Técnicas Retest- all}

Aparentemente, a abordagem seletiva requer mais tempo e recursos para selecionar casos de teste a fim de reduzir o número de casos de teste a serem executados. Um beneficio é alcançado somente se o esforço gasto na seleção for menor que o esforço de executar todos os casos de teste originais, como faz a abordagem retest-all.

Muitos fatores influenciam o custo do teste de regressão. Pode-se distinguí-los em dois conjuntos de fatores: direto e indireto. Direto: inclui custos com as atividades relacionadas ao teste e aos recursos fisicos utilizados para execução dos casos de teste. Indireto: inclui custos com gerenciamento do processo de teste, base de dados para armazenar informaçōes sobre os casos de teste, os resultados da análise estática e históricos de execução. No modelo de custo, proposto por Leung e White (1991), somente os custos diretos são considerados.

$\mathrm{O}$ custo de aplicar um conjunto de casos de teste a um sistema consiste dos seguintes componentes:

$>$ Custo de Análise do Sistema (Ca) - Envolve custos gastos com o estudo da especificação, do projeto e do código do sistema. Esse estudo deve facilitar no julgamento do comportamento do sistema quando da execução dos casos de teste de regressão.

$>$ Custo de Seleção de Teste (Cs) - Envolve custos gastos em selecionar os casos de teste para testar o comportamento atual do sistema. Esse custo depende muito da abordagem escolhida e também da técnica.

$>$ Custo de Execução de Teste (Ce) - Envolve custos gastos em preparar o ambiente para o teste e na execução propriamente dita.

$>$ Custo de Análise dos Resultados (Cr) - Envolve custos gastos em checar o comportamento do sistema sob a execução dos casos de teste. Vários fatores influenciam 
esses custos: tempo para coletar os resultados de saída, tempo para comparar o resultado obtido com o resultado esperado, tempo para registrar esses dados.

Esses componentes dependem de vários fatores e alguns deles estão interrelacionados:

$>$ A abordagem de teste utilizada influencia muito o custo. Ex.: a abordagem que utiliza a técnica de caixa-preta (funcional), que requer apenas a análise da especificação, tem um custo menor que a abordagem que utiliza ambas as técnicas caixa-preta e caixa-branca (estrutural), que requer além da análise da especificação a análise do código fonte.

$>\mathrm{O}$ número de casos de teste também depende da técnica de teste. Ex.: uma técnica que requer que todos os pares de definição-uso sejam executados geralmente necessita de mais casos de teste do que aquela técnica que requer somente que todas as instruções sejam executadas ( $\mathrm{Cs}, \mathrm{Ce}, \mathrm{Cr}$ ).

A complexidade do sistema influencia muito o custo. Quanto maior a complexidade maior é o número de casos de teste e maior é o tempo gasto com a análise do sistema (Ca).

Basicamente, custo de aplicação de uma abordagem $\mathbf{E}$ com relação a um conjunto de casos de teste $\mathrm{T}$ é definido da seguinte forma:

$$
E=C a(T)+C s(T)+C e(T)+C r(T)
$$

na qual, $\mathrm{Cs}, \mathrm{Ce}$ e $\mathrm{Cr}$ são dependentes do número de casos de teste.

Algumas consideraçōes foram feitas para comparar os custos das abordagens retest-all e seletiva:

> Ambas as abordagens são igualmente eficazes.

$>$ A abordagem retest-all não realiza nenhuma análise antes de aplicar todos os casos de teste.

D A abordagem seletiva gasta muito esforço em selecionar um subconjunto de casos de teste para execução. 
Baseando-se nessas observações, a abordagem seletiva é mais econômica do que a abordagem retest-all se o custo para selecionar um subconjunto de casos de teste é menor que o custo para executar e checar casos de teste extras necessários na abordagem retest-all.

Segundo Leung e White (1991), o modelo de custo apresentado é apenas um passo inicial para modelar os esforços gastos nas atividades de teste de regressão. $O$ custo pode ainda ser refinado para incorporar fatores indiretos tais como: gerenciamento, custo para armazenar as informações relacionadas aos casos de teste e custos com desenvolvimento de ferramentas para auxiliar as atividades de teste de regressão. A inclusão desses fatores pode não afetar os resultados já encontrados, no entanto, esses fatores podem ser muito importantes para avaliar a qualidade das abordagens utilizadas para os testes de regressão. Estudos preliminares indicam que quanto maior a complexidade, maior é o número de casos de teste a serem executados, significando assim, um maior tempo gasto. Assim, a abordagem seletiva é mais atrativa que a abordagem retest-all se os custos com gerenciamento forem considerados.

Considerando custos com armazenamento, a abordagem retest-all torna-se mais atrativa que a abordagem seletiva, pois essa abordagem armazena mais dados, tais como: informações da análise estática e dos históricos de cada caso de teste. Essas informações podem dobrar o tamanho da base de dados utilizada para os testes. O custo com desenvolvimento de ferramentas não acarreta maior impacto aos resultados obtidos. Esse custo representa um fator constante para ambas as abordagens.

Leung e White chegaram às seguintes conclusões definindo o modelo de custo descrito:

$>\mathrm{O}$ custo da aplicação de uma abordagem de teste depende de, basicamente, 4 fatores: seleção dos conjuntos de casos de teste de regressão, execução dos conjuntos de casos de teste de regressão, análise dos resultados e razão entre o tamanho do conjunto selecionado com o tamanho do conjunto de casos de teste original.

$>\mathrm{O}$ custo da aplicação de ambas as abordagens depende muito da técnica, da complexidade do programa em teste e do ambiente utilizados.

$>$ Existe uma relação linear entre os valores de $\mathrm{Cs}, \mathrm{Ce}, \mathrm{Cr}$ e o número de casos de teste utilizado.

Duas áreas que garantem estudos são o relacionamento entre a confiabilidade dos produtos de software e as abordagens de teste, e o relacionamento entre o custo do teste e a alcançabilidade da qualidade. Apesar de se desejar uma alta confiabilidade, é igualmente importante relacionar a confiabilidade a um custo razoável. 
Tanto o modelo de custo quanto o framework de avaliação fornecem uma sistemática clara de se avaliar e comparar abordagens e técnicas de teste de regressão. Ambos podem ser utilizados de forma complementar a fim de melhorar a qualidade de se avaliar o teste de regressão.

\subsection{Técnica de Teste de Regressão baseada em Modificação}

O principal objetivo desta técnica é selecionar aqueles casos de teste que revelam um comportamento diferente entre o programa modificado e o programa original. Assim, dado um programa $\mathbf{P}$, seu conjunto de casos de teste de regressão $\mathbf{T}$ e seu programa modificado P', é preciso encontrar $\mathbf{T}^{\prime} \subseteq \mathbf{T}$ tal que:

$$
\forall t \in T, t \in T^{\prime} \leftrightarrow P^{\prime}(t) \neq P(t)
$$

No entanto, encontrar T' não é uma tarefa fácil. Na prática, segundo Wong et al. (1997a), é possível encontrar 'T' somente executando P' com cada caso de teste de regressão em T. Mas essa tarefa não é viável, pois é muito custosa como já mencionado. Entretanto, ao invés de encontrar-se $T^{\prime}$ a partir de $T$, pode-se encontrar $\mathbf{T}^{\prime \prime}, \mathbf{T}^{\prime} \subseteq \mathbf{T}^{\prime}$, incluindo todos os casos de teste em $\mathbf{T}$ que executam o código modificado, ou seja, os casos de teste modification-traversing. Assim:

$\forall t \in T, t \in T^{\prime \prime} \leftrightarrow t$ executa código em $P$ que foi modificado ou removido para gerar $P$ ' ou código em $P$ no qual novo código foi adicionado para gerar $P^{\prime}$

Portanto, T" é um subconjunto de $\mathrm{T}$ baseado em modificação. A Figura 2.2 ilustra o esquema apresentado. Apesar do conjunto T" ser possuir todos os casos de teste que passam por alguma modificação, ele pode conter, ainda, casos de teste que não revelam um comportamento diferente entre P e P', sendo assim, não preciso. Assim, a seguinte questão: “Que procedimentos ou mecanismos podem ser aplicados para reduzir o número de casos de teste visando a eliminar aqueles casos de teste que não revelam comportamento diferente?" Os mecanismos de minimização e priorização são propostos para solucionar essa questão. 


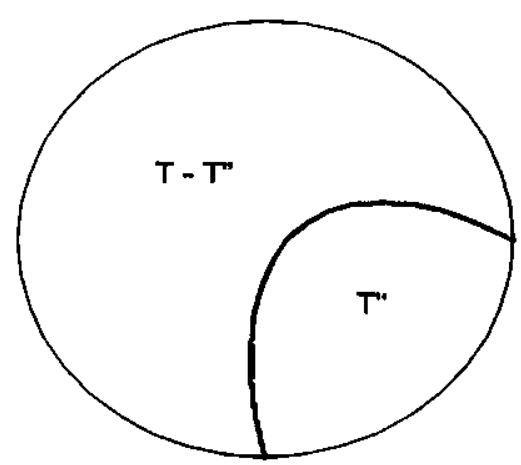

Té o conjunto de teste de

regressão original que

contém todos os testes

Figura 2.2 - Conjunto T" baseado em modificação.

O mecanismo de minimizacão encontra um subconjunto mínimo de casos de teste de regressão que preserve a cobertura com respeito a um dado critério a partir de $\mathbf{T}$. $\mathbf{O}$ mecanismo de prioridades seleciona casos de teste de regressão utilizando o mecanismo de minimização de acordo com a análise de cobertura de cada caso de teste segundo um dado critério. Sob esse cenário, testadores podem selecionar os casos de teste no topo da lista de prioridades. Tal flexibilidade não aparece em nenhuma outra técnica de revalidação seletiva. $\mathrm{O}$ uso de ambos os mecanismos oferece algumas vantagens:

Reduz a quantidade de esforço requerido para testar o conjunto minimizado.

Aumentam as chances de se escolherem casos de teste que produzam saídas diferentes na antiga e na nova versão do programa.

$>$ Diminuem as chances de inclusão de casos de teste que falhem quando da distinção entre o antigo e o novo programa.

Para selecionar o conjunto de casos de teste de regressão mínimo e priorizado a partir de um conjunto $\mathbf{T}$ adequado, 2 passos são realizados:

Construção de um superconjunto de todos os casos de teste de regressão que devem ser utilizados.

Se necessário, utilizar a minimização e priorização.

Esses dois passos provêem um guia sobre quais casos de teste devem ser utilizados para executar as modificações realizadas. A aplicação desses dois passos está ilustrada na Figura 2.3 a seguir. 
Primeiramente, os casos de teste modification-traversing, conjunto T", são selecionados a partir do conjunto de casos de teste de regressão T disponível e das modificações realizadas. Em seguida, se necessário, o mecanismo de minimização e priorização são aplicados. Cada mecanismo gera um subconjunto de teste de regressão diferente a partir do conjunto T”. Note que esses mecanismos não são aplicados seguidos um do outro, e sim de forma separada. No entanto, um mecanismo poderia ser utilizado como complemento do outro a fim de reduzir ainda mais o número de casos de teste a serem reexecutados. Após esse processo, caso seja necessário, novos casos de teste são gerados.

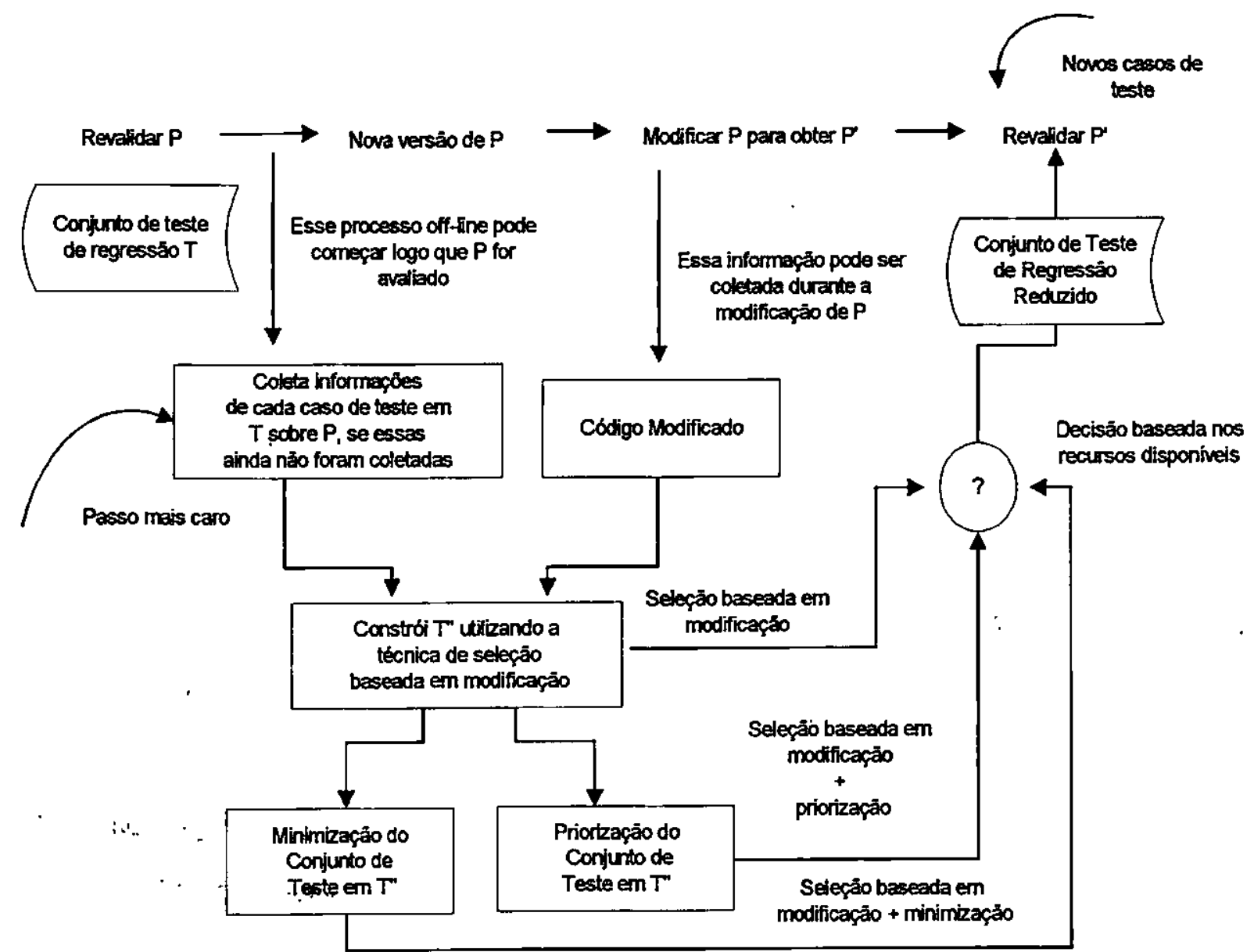

Figura 2.3 - Seleção dos casos de teste de regressão utilizando um processo off-line (Wong et al.,1997a).

A fim de avaliar a técnica baseada em Modificação, Wong et al. conduziram um experimento utilizando-se o programa $\mathrm{SPACE}^{3}$, desenvolvido pela Agência Espacial Européia, e a ferramenta de teste $A T A C$ (descrita na Seção 2.6.1). Para realização desse estudo de caso,

\footnotetext{
${ }^{3}$ O programa SPACE permite que sc possa descrever a configuração de um vetor de antenas utilizando-sc de uma linguagem de alto nível.
} 
obteve-se uma lista de erros, Tabela 2.1, que foi elaborada durante as fases de teste e integração do SPACE. Com base nessa lista de erros, dez programas com defeitos foram gerados. Para cada um desses programas com defeitos, conjuntos de casos de teste de regressão baseados em modificação foram gerados, e a partir desses conjuntos baseados em modificação, conjuntos minimizados e priorizados foram selecionados seguindo o processo ilustrado na Figura 2.3. Os conjuntos de casos de teste de regressão baseados em modificação foram selecionạdos a partir de um conjunto original de 1000 casos de teste.

O mecanismo de minimização utilizado no experimento foi implementado na ferramenta ATAC. Já o mecanismo de priorização utilizou-se da cobertura dos critérios de teste todos-usos, cobertura de blocos e cobertura de decisões dos programas para selecionar os conjuntos priorizados. Casos de teste modification-traversing foram sendo inseridos no topo da lista de prioridades à medida que esses casos melhoravam a cobertura com relação a cada um dos critérios. Utilizando-se dessa lista de prioridades, os conjuntos priorizados para cada defeito foram definidos com os $\mathbf{n}$ primeiros casos de teste da lista. $\mathrm{O}$ valor de $\mathbf{n}$, segundo Wong et al. é desconhecido, pois não se sabe quantos casos de teste que estão na lista de prioridades devem ser selecionados. Assim, no experimento conduzido por Wong et al. foi definido que o valor máximo de $\mathbf{n}$ era o número de casos de teste selecionados pelo mecanismo de minimização Foram selecionados três conjuntos priorizados diferentes para cada defeito: um conjunto com 1/3 do número de casos de teste dos conjuntos minimizados, outro com $2 / 3$ desse número e outro com $3 / 3$ desse número.

A partir desse experimento realizado, Wong et $\dot{a} l$. concluíram:

Um teste de regressão é um trade-off entre o número de casos de teste de regressão necessários e seu custo.

$>$ Quanto maior o número de casos de teste de regressão, mais completo é o processo de revalidação, no entanto, esse processo requer muitos recursos e, muitas vezes, pode não ser prático.

Executar poucos casos de teste pode ser mais barato, mas pode comprometer a garantia de que as novas funcionalidades sejam validadas e verificadas, ou seja, pode comprometer a eficácia e a eficiência do teste de regressão. 
Assim, a utilização da técnica baseada em modificação, juntamente com a minimização e priorização, permite a escolha de um conjunto eficaz para realização dos teste de regressão com um menor custo.

Tabela 2.1 - Lista de erros utilizada para gerar os programas com defeitos.

Equacōos incorretas ou insuficientes

Alteraç̄es nos comandos de condiç̃es

Dados acessados ou armazenados incorretamente

Variáveis incorretas

\subsection{Técnica de Teste de Regressão baseada em Mutação Seletiva}

Uma característica especial da seleção de casos de teste de regressão utilizando mutação seletiva é que, como mostra a Figura 3.4, a seleção pode ser inicializada logo que a nova versão do programa é finalizada. A solução apresentada por esta técnica depende somente do programa original, do conjunto dos casos de teste de regressão e do conjunto de operadores de mutação. Ou seja, a seleção dos casos de teste pode ser realizada por um processo off-line antes que qualquer modificação seja realizada.

A principal estratégia desta técnica é examinar como um critério $\mathbf{C}$ pode ser utilizado no teste de regressão a fim de auxiliar testadores a determinarem quais casos de teste devem ser selecionados ou têm maior prioridade para revalidar as funcionalidades herdadas da versão anterior do programa, e quais devem ser omitidos ou têm baixa prioridade para tal revalidação. A qualidade de $\mathbf{C}$ e dos casos de teste selecionados pode ser medido com o auxílio de duas métricas:

Redução do número de casos de teste a serem reexecutados.

Redução da eficácia do número de defeitos que podem ser revelados por $\mathbf{T}$ que não foram baseadas em $\mathbf{C}$.

A mutação seletiva, como já mencionado, consiste em selecionar um conjunto de operadores de mutação para encontrar o conjunto minimizado de casos de teste a serem aplicados durante os testes de regressão. Estudos empíricos demonstram que a mutação seletiva é bastante eficiente em revelar defeitos e pode contribuir para a redução dos custos aplicados nas atividades de teste (Mathur e Wong, 1994; Offut et al., 1996a, Offut et al., 1996b, Wong e Mathur, 1995a; Wong e Mathur, 1995b; Wong et al., 1997c). 
A técnica é aplicada da seguinte maneira (Figura 2.4). Logo que o programa é finalizado, um conjunto de teste de regressão é definido a partir de um conjunto de operadores de mutação e do conjunto de casos de teste disponível. Se um caso de teste desse conjunto melhora o escore de mutação com relação à mutação seletiva, esse caso de teste é inserido no conjunto de teste de regressão reduzido. Esse processo continua até que todos os casos de teste sejam examinados. Ao final do processo um conjunto de casos de teste de regressão reduzido é selecionado. Se necessário, novos casos de teste são gerados.

Segundo Wong et al. (1997b), o custo do teste de regressão pode ser medido de várias maneiras: tempo necessário para executar os casos de teste e tempo despendido pelo testador em analisar os resultados obtidos. Ambos os custos são proporcionais ao número de casos de teste a serem reexecutados.

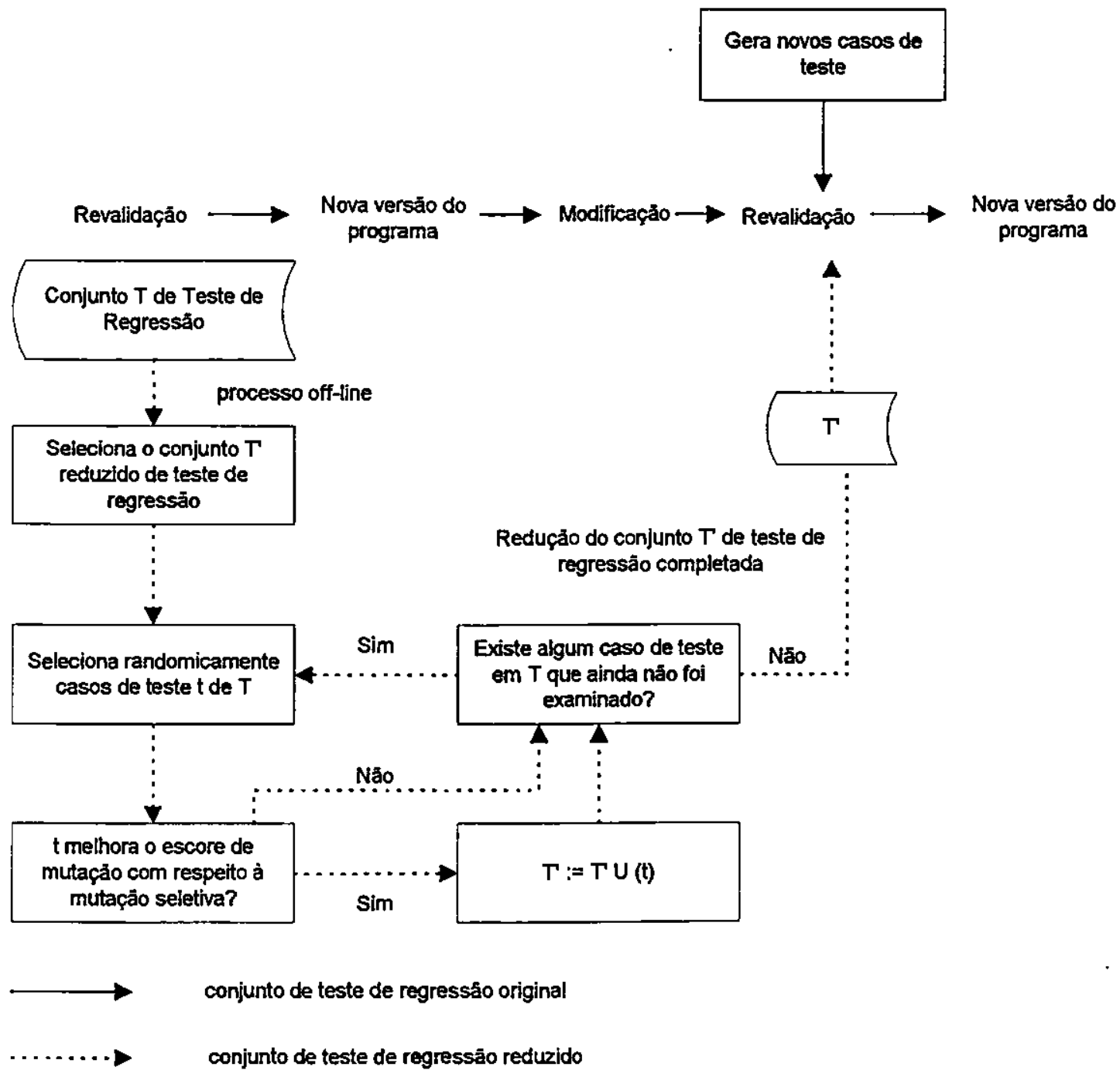

Figura 2.4 - Redução do conjunto de teste de regressão (Wong et al. 1997b). 
Para avaliar a aplicação da técnica baseada em Mutação Seletiva, Wong et al. conduziram um experimento utilizando-se de um conjunto de programas UNIX. Os seguintes passos foram realizados para avaliar a técnica:

\section{Passo 1 - Preparação dos dados dos programas}

Os programas utilizados foram nove primitivas do UNIX, vide Tabela 2.2. Para cada um desses programas, uma lista de erros e um conjunto de casos de teste de regressão foram estabelecidos. A Tabela 2.3 apresenta a lista de erros utilizada. Baseando-se nessa lista de erros, os programas com defeitos foram gerados.

Tabela 2.2 - Programas UNIX utilizados nos experimentos.

\begin{tabular}{|c|l|}
\hline Programas & Imprime calendário de um ano ou mês especifico \\
\hline Cal & Reporta delimitadores desbalanceados \\
\hline Checkeq & Filtra arquivos tratando caracteres especiais \\
\hline Comm & Seleciona ou rejeita linhas comuns de dois arquivos classificados \\
\hline Crypt & Criptografa ou decriptografa arquivos utilizando password \\
\hline Look & Encontra palavras em um sistema ou linhas \\
\hline Sort & Classifica e realiza merge em arquivos \\
\hline Spline & Interpola pontos \\
\hline Tr & Traduz caracteres \\
\hline Uniq & Reporta ou remove linhas adjacentes duplicadas \\
\hline
\end{tabular}

Tabela 2.3 - Tipos de defeitos utilizados nos experimentos.

\begin{tabular}{|l|l|}
\hline Caminhos removidos & Troca de operador relacional \\
\cline { 2 - 2 } & Troca de operador lógico \\
\hline \multirow{4}{*}{ Declaraçōes incorretas } & Inicialização incorreta \\
\cline { 2 - 2 } & Constante incorreta \\
\cline { 2 - 2 } & Precedência incorreta \\
\cline { 2 - 2 } & Referência de vetor incorreta \\
\cline { 2 - 2 } & Operação de ponteiro incorreta \\
\hline & Troca de mesmo tipo de variável \\
\cline { 2 - 2 } & Troca de operador aritmético \\
\hline & Miscelânea \\
\hline \multirow{2}{*}{ Declaraçöes removidas } & Remoção completa de declaração \\
\cline { 2 - 2 } & Remoção parcial de declaração \\
\hline Número incorreto de iteraçöes & \\
\hline Predicados removidos & \\
\hline
\end{tabular}




\section{Passo 2 - Seleção dos Operadores de Mutação}

Doze operadores de mutação da ferramenta PROTEUM foram selecionados, de acordo com estudos realizados por (Offut et al., 1996b; Wong e Mathur, 1995a; Wong e Mathur, 1995b; Wong et al., 1997c), para que os mutantes fossem gerados para cada programa. A Tabela 2.4 mostra os operadores de mutação utilizados para cada critério de mutação seletiva. $O$ Apêndice A apresenta detalhes sobre cada um desses operadores de mutação.

Mutantes a nível estrutural e a nível declarativo foram gerados e avaliados. Mutantes estruturais modificam a estrutura do programa e estão relacionados com fluxo de controle e/ou fluxo de dados de algumas variáveis do programa original. Mutantes a nível declarativo realizam pequenas mudanças sintáticas nas declarações sem alterar a estrutura.

Tabela 2.4 - Operadores de mutação e critérios de mutação seletiva.

\begin{tabular}{|c|c|c|}
\hline & Operador de Mutaçó & Critério \\
\hline MUT-A & VDTR, VTWD & Declarativo \\
\hline MUT-B & SSDL & Estrutural \\
\hline MUT-C & SMVB, SBRn, SCRn & Estrutural \\
\hline MUT-D & Vprr, Vtrr, CRCR & Declarativo \\
\hline MUT-E & OLLN, OLNG, ORRN & Estrutural \\
\hline
\end{tabular}

\section{Passo 3 - Seleção dos Conjuntos de Casos de Teste de Regressão Reduzidos}

O esquema da Figura 3.4 ilustra como foi realizada a seleção dos conjuntos de casos de teste de regressão reduzido. A partir de um conjunto de casos de teste de regressão $\mathbf{T}$, selecionaram-se $t$ casos de teste aleatoriamente, $t \in T$, gerando um conjunto reduzido de casos de teste de regressão $\mathbf{T}^{\prime}$.Se o caso de teste $\mathbf{t}$ melhorasse o escore de mutação com relação à mutação seletiva, este era incluído no conjunto $\mathbf{T}^{\mathbf{\prime}}$. Esse processo foi realizado até que todos os casos de teste foram avaliados. Uma vez incluido no conjunto $\mathbf{T}$, o caso de teste não é excluido devido à inclusão de qualquer outro caso de teste.

Para cada programa foram gerados cinco conjuntos de casos de teste $\left(T_{n}\right)$ a partir do conjunto de casos de teste original $\mathrm{T}$ em diferentes ordens. Para cada um desses conjuntos foram selecionados cinco subconjuntos de casos de teste $\left(T_{n}\right)$ para serem utilizados no teste de regressão. $\mathrm{O}$ primeiro conjunto, $\mathrm{T}_{0}$, é composto por todos casos de teste disponíveis ordenados de forma crescente, enquanto que os demais conjuntos, $T_{1}, T_{2}, T_{3}$ e $T_{4}$ são permutações do conjunto $\mathrm{T}_{0}$ obtidas aleatoriamente. Cada caso de teste recebe uma numeração para ser executado pela 
ferramenta PROTEUM. Esse é a numeração que foi permutada para gerar os conjuntos $\mathrm{T}_{1}, \mathrm{~T}_{2}, \mathrm{~T}_{3}$ e $\mathrm{T}_{4}$.

A técnica baseada em mutação seletiva mostrou-se bastante promissora, pois permitiu com uma grande redução dos números de casos de teste a serem reexecutados sem comprometer a eficácia dos conjuntos em relevar os defeitos de cada programa UNIX. Além disso, um conjunto minimizado de casos de teste de regressão também contribui para a redução dos custos sem comprometer significativamente a eficácia das atividades de teste de regressão (Wong et al., 1997a; Wong et al., 1997b).

\subsection{Ferramentas de Teste e Estudos Empíricos}

As atividades de teste de software são bastante custosas, demandando muitos esforços de desenvolvedores, programadores e testadores, como já mencionado. Assim, com o intuito de reduzir os custos aplicados nessas atividades, diversas ferramentas de teste foram desenvolvidas. Com o auxilio dessas ferramentas, diversos estudos empíricos também têm sido realizados visando a estabelecer estratégias e critérios que auxiliem na redução desses esforços. Muitos desses estudos são relevantes para este trabalho, portanto, são sintetizados nessa seção juntamente com algumas ferramentas de teste de software.

\subsubsection{Ferramentas de Teste}

A seguir uma sintese de algumas ferramentas de teste é apresentada. As ferramentas de teste PROTEUM, PROTEUMMM, que apóiam as atividades de teste durante o desenvolvimento do software, são descritas com mais detalhes, pois foram utilizados nos experimentos descritos no Capítulo 3. Outras ferramentas, como: ATAC, POKETOOL, RePOKETOOL, DEJAVU são descritas mais sucintamente.

\section{A ferramenta PROTEUM}

A PROTEUM (Delamaro, 1993) apóia o critério Análise de Mutantes e está configurada para funcionar em estações SUN sob o ambiente OPENWINDOWS. A ferramenta apresenta interface gráfica e oferece recursos para a execução das seguintes operações: definição de casos de teste, execução do programa em teste, seleção dos operadores de mutação que serão utilizados para gerar os mutantes, geração dos mutantes, execução dos mutantes com os casos de teste 
definidos, análise dos mutantes vivos e cálculo do escore de mutação. As funções implementadas na PROTEUM possibilitam que alguns desses recursos sejam executados automaticamente, enquanto que para outros são fornecidas facilidades para que o testador possa realizá-los. A PROTEUM destina-se a teste de programas escritos na linguagem $\mathrm{C}$, entretanto, é uma ferramenta multilinguagem que permite a configuração para outras linguagens.

A ferramenta possui 71 operadores de mutação divididos em quatro classes: mutação de comandos, mutação de operadores, mutação de variáveis e mutação de constantes. Essa divisão permite a escolha dos operadores de mutação de acordo com a classe de erros que se deseja revelar, de modo que a geração dos mutantes possa ser feita em etapas ou que se possa dividir a atividade de teste entre vários testadores (Vincenzi, 1998).

A ferramenta permite ao testador avaliar a adequação de um conjunto de casos de teste $\mathbf{T}$ para um determinado programa $\mathbf{P}$ e com o resultado dessa avaliação o testador pode melhorar $o$ conjunto $\mathbf{T}$ visando a satisfazer o critério Análise de Mutantes.

As operações mínimas suportadas pela ferramenta são:

Manipulação de casos de teste: execução, inclusão e exclusão.

Manipulação de mutantes: geração, execução e análise.

Análise de adequação: escore de mutação e relatórios estatísticos.

A ferramenta PROTEUM pode importar casos de teste da ferramenta POKE-TOOL, de sessões de teste dela mesma ou pode gerar casos de teste aleatoriamente. As informações geradas pela PROTEUM são armazenadas em uma base de dados composta por: uma base com informações sobre os casos de teste utilizados, uma base com informações sobre os mutantes e alguns arquivos intermediários que descrevem o programa em teste. Uma sessão de teste caracteriza-se de uma seqüência de operações realizadas sobre essas bases de dados.

A implementação da ferramenta PROTEUM facilitou a condução de trabalhos comparativos entre critérios de teste funcional, estrutural e baseados em erros, assim como o estudo e a avaliação de estratégias de aplicação de variantes da Análise de Mutantes (Mutação Restrita) a exemplo de Mathur e Wong (1993), no contexto de programas C, linguagem de muito interesse no ambiente UNIX. Assim, viabiliza a condução de benchmarks que visem a demonstrar a factibilidade desse critério, avaliar o custo e a sua eficiência e avaliar o aspecto complementar deste com outros critérios. 


\section{A ferramenta PROTEUM/M}

A PROTEUM/IM apóia o critério Interface Mutation. A ferramenta está disponível para os sistemas operacionais SunOS e Linux e configurada para o teste de programas escritos na linguagem C, sendo também uma ferramenta multilinguagem (Delamaro, 1997a). A PROTEUM/IM possui 33 operadores de mutação divididos em dois grupos. O primeiro grupo aplica mutações em um módulo B chamado por um outro módulo A, enquanto o segundo grupo aplica mutações no ponto onde B é chamado dentro de A.

A ferramenta permite ao testador avaliar a adequação de um conjunto de casos de teste $\mathbf{T}$ para um determinado programa $\mathbf{P}$ e com o resultado dessa avaliação o testador pode melhorar o conjunto $\mathbf{T}$ visando a satisfazer o critério Mutação de Interface.

As operações mínimas suportadas pela ferramenta são as mesmas suportadas pela PROTEUM:

Manipulação de casos de teste: execução, inclusão e exclusão.

Manipulação de mutantes: geração, execução e análise.

Análise de adequação: escore de mutação e relatórios estatísticos.

O Quadro 2.1 mostra quais são os programas que compõem as ferramentas PROTEUM/IM e PROTEUM (Vincenzi, 1998). Esses programas podem ser utilizados em conjunto na condução de uma sessão de teste. Os programas disponíveis estão divididos em dois grupos. O primeiro é composto por programas básicos que agem diretamente na base de teste que caracteriza a sessão. O segundo é composto por programas utilitários que utilizam os programas básicos para realizar algumas operações durante uma sessão de teste (Delamaro e Maldonado, 1997c).

A execução desses programas pode ser feita de duas formas: diretamente na linha de comando através de scripts ou de forma transparente para o usuário através da interface gráfica. A interface gráfica facilita a condução de uma sessão de teste no caso do usuário ser iniciante. Os recursos de visualização dos casos de teste e dos mutantes são melhores com a interface gráfica, tornando a condução da sessão de teste mais fácil. No entanto, esse tipo de interface depende muito da interação do usuário, sendo, assim, menos flexível do que chamar diretamente os programas (Vincenzi, 1998). A chamada direta aos programas, na forma de scripts, reduz o número de interações com as ferramentas e possibilita a execução de longas sessões de teste em batch, nas quais o usuário pode construir um programa especificando as tarefas a serem 
realizadas e a ferramenta executa esse programa, reduzindo, assim, o tempo gasto na atividade de teste. Entretanto, se o usuário optar por utilizar scripts, a elaboração de scripts exige um esforço de programação e um completo domínio tanto dos conceitos sobre teste baseado em mutação quanto dos próprios programas que compõem a ferramenta.

Quadro 2.1 - Programas que compõem a PROTEUM/M e a PROTEUM.

\begin{tabular}{l|l}
\hline \multicolumn{2}{c}{ Programas Básicos } \\
\hline li & Transforma um programa C para uma linguagem intermediária (LI) \\
\hline pi2nli & Ccria o grafo de programa e adiciona informações sobre os nós na LI \\
\hline tcase & $\begin{array}{l}\text { Ccria e manipula arquivos de teste de programas, os quais descrevem as características } \\
\text { gerais da sessão de teste }\end{array}$ \\
\hline muta & Ccria e manipula a base de dados de casos de teste \\
\hline exemuta & Cria e manipula a base de dados dos mutantes \\
\hline opmuta & Constrói o código fonte dos mutantes e os executa; ativa/desativa mutantes \\
\hline report & Aplica os operadores de mutação no programa original, criando descritores de mutação \\
\hline \multicolumn{2}{|c}{ Pria um relatório sobre a efetividade dos casos de teste } \\
\hline test-view & Cria uma nova sessão de teste \\
\hline tcase-add & Insere um caso de teste interativamente \\
\hline muta-gen & Gera descritores de mutação e os insere na base de dados dos mutantes \\
\hline muta-view & Permite visualizar e analisar mutantes
\end{tabular}

\section{A ferramenta POKE-TOOL}

A POKE-TOOL (Potential Uses Criteria Tool for Program Testing) (Chaim, 1991) é uma ferramenta de teste que apóia os critérios estruturais Potenciais-Usos, Todos-Nós e TodosArcos. A versão inicial da ferramenta utiliza unidades de programa escritos na linguagem $\mathrm{C}$, entretanto, a ferramenta possui a característica de multilinguagem.

A ferramenta é orientada à sessão de trabalho, na qual o usuário entra com o programa a ser testado, com o conjunto de dados de teste e seleciona um dos critérios suportados.

A $P O K E-T O O L$ possui nove funções para fornecer os dados acima e são mostradas no Quadro 2.2. 
Quadro 2.2 - Funç̃es implementadas pela ferramenta POKE-TOOL.

\begin{tabular}{l|l}
\hline Grafo de Fluxo de Controle & $\begin{array}{l}\text { gera o grafo de fluxo de controle (GFC) do programa em teste. O } \\
\text { GFC é gerado a partir de uma versão em linguagem } \\
\text { intermediária (LD) produzida pela ferramenta a partir do } \\
\text { programa em teste }\end{array}$ \\
\hline Cálculo dos Arcos Primitivos & $\begin{array}{l}\text { calcula os arcos primitivos do GFC que irão servir para geração } \\
\text { da versão instrumentada do programa em teste }\end{array}$ \\
\hline $\begin{array}{l}\text { Extensão do Grafo de Fluxo de } \\
\text { Controle }\end{array}$ & gera o Grafo Def \\
\hline Interface Gráfica & apresenta o GFC (Vilela, 1994; Vilela et al., 1996) \\
\hline Instrumentação & $\begin{array}{l}\text { insere comandos de escrita (pontas de prova) no programa em } \\
\text { teste para cada nó do GFC que produzem um trace da execução } \\
\text { dos casos de teste. }\end{array}$ \\
\hline Compilador Selecionado & $\begin{array}{l}\text { compilador da linguagem fonte na qual o programa em teste foi } \\
\text { implementado }\end{array}$ \\
\hline Execução do Programa & controla a execuçăo do programa em teste \\
\hline Construçăo do Grafo(i) & $\begin{array}{l}\text { gera o conjunto de caminhos e associaçóes requeridas para } \\
\text { satisfazer os critérios Potenciais-Usos }\end{array}$ \\
\hline Avaliação & $\begin{array}{l}\text { verifica se o conjunto de caminhos ou associaçães executados } \\
\text { satisfaz o critério selecionado, fazendo um relacionamento de } \\
\text { cobertura }\end{array}$
\end{tabular}

\section{A ferramenta $A T A C$}

A ATAC (Automatic Testing Analysis tool in C) realiza testes baseando-se na cobertura do fluxo de dados de programas escritos na linguagem C. Dado um programa para teste, a $A T A C$ encontra o seu conjunto de atributos testáveis tais como blocos, comandos de decisão, p-uso, cuso e todos-usos, e instrumenta o programa para armazenar informações através de trace durante a execução do teste. A $A T A C$ utiliza esse trace para gerar os casos de teste (Wong et al., 1997a).

A ferramenta conduz a seleção dos casos de teste baseando-se nas modificações realizadas e encontra os conjuntos minimizados e os conjuntos priorizados de casos de teste. Para o conjunto priorizado, a ferramenta seleciona casos de teste que melhoram a cobertura com respeito a um critério. Para o conjunto minimizado, a ferramenta utiliza um algoritmo com redução para encontrar o subconjunto minimizado baseando-se nos testes examinados.

\section{As ferramentas DEJAVU e RePOKE-TOOL}

Considerando o teste de regressão, algumas técnicas são apoiadas por ferramentas. Rothermel e Harrold (1997 e 1998) propuseram uma técnica de teste de regressão que se baseia na cobertura para selecionar casos de teste e é implementada pela uma ferramenta DEJAVU. Granja (1997) desenvolveu uma técnica seletiva que se baseia no teste estrutural mais especificamente nos critérios Potenciais-Usos que apoiada pela ferramenta Re-POKETOOL. A 
Re-POKETOOL (Regression Testing support for Potencial-Uses Criteria Tool) pode ser aplicada em unidades que sofreram algum tipo de modificação. A ferramenta seleciona casos de teste para serem utilizados no teste de regressão a partir dos elementos requeridos para o teste estrutural que foram modificados ou inseridos após a fase de manutenção.

\subsubsection{Estudos Empíricos}

Os critérios de adequação fornecem mecanismos para seleção sistemática de um subconjunto de casos de teste para um programa, de modo que esse subconjunto seja eficaz na deteç̧ão de defeitos. A partir de um dado critério de adequação pode-se obter um número infinito de subconjuntos de casos de teste adequados ao programa em teste. Dependendo do critério e da forma utilizada na geração dos casos de teste, pode-se chegar a um conjunto bastante grande, dificil de ser manipulado e que aumenta o custo na condução da atividade de teste (Vincenzi, 1998).

"A minimização de conjuntos de casos de teste tem o objetivo de, a partir de um conjunto de casos de teste $\mathbf{T}$, gerar um conjunto $\mathbf{T}_{\min }$ com o mesmo grau de adequação de $\mathbf{T}$, geralmente com um tamanho menor. A redução do tamanho do conjunto $\mathbf{T}$ é obtida pela eliminação de casos de teste redundantes e obsoletos. A minimização procura beneficiar os testes de regressão, como também fornecer um mecanismo mais real para avaliação do custo de aplicação de um critério. Muitas vezes esse tipo de avaliação é que determina o uso ou não de um critério para a condução dos testes." (Souza, 1996).

As estratégias que realizam minimização de conjuntos de casos de teste, além de permitirem redução de custos nas atividades de teste, mais particularmente o critério Análise de Mutantes, também possibilitam a obtenção de parâmetros mais reais para quantificar o custo de aplicar um critério durante o desenvolvimento de estudos empíricos. Entretanto, a redução do conjunto é uma tarefa dificil na prática, uma vez que, segundo Garey e Johnson (1979), o problema de encontrar em conjunto mínimo é NP-Completo.

Uma contribuição relevante do trabalho de Souza (1996), foi a implementação de um módulo de minimização de casos de teste, denominado MINIMIZE, para a ferramenta PROTEUMI.2-C. Com base nesse módulo, um experimento foi realizado visando avaliar a redução no tamanho dos conjuntos e também o efeito dessa redução na eficácia em revelar erros. Observou-se que a minimização proporciona uma redução significativa no tamanho dos conjuntos de casos de teste e com relação à eficácia dos conjuntos mínimos houve, em alguns 
casos, uma pequena redução da eficácia. O módulo MINIMIZE foi modificado para se integrar a nova versão da ferramenta, PROTEUM1.4.1. No entanto, sua funcionalidade não foi alterada, mas sim a estrutura de dados. Essa adaptação do módulo foi realizada por Tullio (1999) em seu trabalho de iniciação científica.

O módulo MINIMIZE tem como principal objetivo obter um subconjunto de casos de teste a partir do conjunto inicial de casos de teste adequados ao critério Análise de Mutantes. A implementação do módulo está baseada no algoritmo proposto por Harrold et al. (1993) o qual apresenta a vantagem de poder ser aplicado a qualquer critério de teste que contenha associações entre os requisitos de teste e os casos de teste. A minimização é realizada sobre uma sessão de teste já existente, composta de um conjunto de casos de teste adequado ao critério Análise de Mutantes.

As principais características do módulo MINIMIZE são:

Sendo um módulo integrante da ferramenta PROTEUM, este deve seguir o mesmo padrão de desenvolvimento e utilizar, quando pọssível, as bibliotecas já definidas. Além disso, desempenha função específica e funciona de forma isolada dos demais módulos, se relacionando somente através das bases de dados.

Permite que seja mantido no conjunto minimizado um determinado número de casos de teste. Essa característica permite, por exemplo, manter os casos de teste que revelem erros.

$>$ A chamada ao módulo pode ser feita via shell scripts.

Uma contribuição importante para a minimização de custos da aplicação dos critérios Análise de Mutantes e Mutação de Interface foram os trabalhos realizados por Barbosa e Vincenzi (1998; 1998). Barbosa desenvolveu um algoritmo que permite definir um conjunto de operadores essenciais para a Linguagem C, denominado Essencial. Esse algoritmo estabelece um conjunto essencial de operadores considerando apenas o critério Análise de Mutantes. Para validar o algoritmo definido, utilizaram-se dois grupos distintos de programas: um conjunto de programas UNIX e um conjunto de programas em C (27) definidos por Maldonado (1991). Para ambos os conjuntos de programas, o conjunto essencial obtido apresentou resultados bastante significativos quanto à redução de custos, com um decréscimo muito pequeno no grau de adequação em relação ao critério Análise de Mutantes (Barbosa, 1998). 
Vincenzi desenvolveu uma estratégia de aplicação dos operadores de mutação, tanto para - critério Análise de Mutantes quanto para o critério Mutação de Interface. Essa estratégia estabelece 0 teste incremental que combina ambos os critérios de mutação. Com 0 desenvolvimento dessa estratégia, além de se objetivar a minimização dos custos da aplicação de ambos os critérios de mutação, pretendeu-se comparar a adequação entre os critérios Análise de Mutantes e Mutação de Interface. A estratégia estabelecida foi validada por meio de experimentos realizados com os mesmos dois grupos de programas utilizados por Barbosa em seus experimentos.

\subsection{Considerações Finais}

Neste capítulo foram apresentadas as principais técnicas de teste de software e as principais características de cada técnica, além de diversas ferramentas que apoiam a aplicação dessas técnicas.

Tão importante quanto o teste realizado durante o processo de desenvolvimento de software é o teste de regressão, pois o software evolui constantemente e deve ser testado visando a garantir que suas funcionalidades não foram afetadas adversamente com as modificações. Pode-se perceber que cada técnica baseia-se em conjuntos diferentes de informações para derivar os requisitos de teste. Devido a esse fato, estudos empíricos são necessários visando a comparar a eficácia da aplicação de cada uma dessas técnicas.

Assim como o teste de software tradicional, as atividades de teste de regressão também demandam muito custo. Visando a reduzir esses custos, vários pesquisadores têm proposto diversas técnicas que selecionam um subconjunto de casos de teste, a partir do conjunto original, para realizar os testes de regressão. Além disso, pesquisadores têm estudado continuamente mecanismos para avaliar e comparar essas diversas técnicas.

O próximo capitulo apresenta os experimentos realizados com as Técnicas de teste de regressão baseadas em Modificação e Mutação Seletiva. 



\section{Capítulo 3}

\section{Aplicação e Avaliação das Técnicas de Teste de Regressão}

\subsection{Considerações Iniciais}

A realização de estudos teóricos e empíricos é importante para fornecer conhecimento e subsídios para o estabelecimento de estratégias de teste de software, seja essa atividade realizada durante a fase de desenvolvimento ou durante a fase de manutenção. Assim como o teste de software realizado durante o desenvolvimento é apoiado por técnicas e critérios de teste, o teste de regressão realizado durante a fase de manutenção também deve ser apoiado por técnicas de teste que visam a selecionar um conjunto de casos de teste a serem reexecutados para avaliar a confiabilidade das modificações realizadas. Por meio de estudos empíricos, é possível avaliar o custo e os beneficios da aplicação das diversas técnicas de teste de regressão existentes na literatura. Essa avaliação visa a facilitar a escolha de uma dessas técnicas quando da realização das atividades de teste de regressão.

Assim, neste capítulo são descritos os estudos empíricos realizados com os programas UNIX e o programa SPACE utilizando-se as técnicas de teste de regressão baseada em Modificação e Mutação Seletiva. Neste trabalho a técnica baseada em Modificação foi aplicada nos programa UNIX, enquanto que a técnica baseada em Mutação Seletiva foi aplicada ao programa SPACE, ao contrário do trabalho realizado por Wong et al. como ilustrado na figura do Capítulo 1. O framework definido por Rothermel e Harrold (1996) foi utilizado para avaliar essas técnicas de teste de regressão. 


\subsection{Descrição Geral dos Experimentos}

Rothermel e Harrold (1997) definem duas maneiras possiveis de se realizarem estudos empirícos relacionados ao teste de regressão: 1. Considerar que as versões modificadas são tentativas mal sucedidas de se evoluir o programa original; ou 2. Considerar o programa original como sendo a versão correta de uma família de versões incorretas (modificadas). Para estes experimentos, a primeira maneira foi utilizada.

O ambiente dos experimentos é constituidos dos seguintes elementos:

$>$ Programas em teste.

$>$ Mecanismos e ferramentas.

$>$ Métricas.

Os programas utilizados nos experimentos foram os programas UNIX e o programa SPACE descritos no Capitulo 2. Os mecanismos e ferramentas utilizados foram: ferramenta PROTEUM, ferramenta PROTEUMIM e o módulo de minimização MINIMIZE, também no Capítulo 2. As métricas utilizadas para a medição dos resultados, além das características do framework de Rothermel e Harrold descritas no Capitulo 2, foram as seguintes (Wong et al., 1997a; Wong et al., 1997b):

$>$ Redução de Tamanho: [1 - $\left(\mathrm{n}^{\circ}\right.$ de casos selecionados em $\mathrm{Ts} / \mathrm{n}^{\circ}$ de casos em $\left.\left.\mathrm{T}\right)\right]$ * 100

$>$ Reduçäo da Efícácia: $\left[1-\left(n^{\circ}\right.\right.$ de defeitos revelados por $\mathrm{T} s / \mathrm{n}^{\circ}$ de defeitos revelados por $\left.\left.\mathrm{T}\right)\right]$ $* 100$

$>$ Eficácia: ( $\mathrm{n}^{\circ}$ de defeitos revelados por $\mathrm{T} s / \mathrm{n}^{\circ}$ de defeitos disponíveis)

$>$ Precisão: ( $\mathbf{n}^{\circ}$ de casos de teste em Ts que revelam defeitos $/ \mathbf{n}^{\circ}$ casos de teste em Ts) * 100

$>$ Recall: $\left(\mathbf{n}^{\circ}\right.$ de casos de teste em $\mathrm{Ts}$ que revelam defeitos $/ \mathbf{n}^{\circ}$ casos de teste em $\left.\mathrm{T}^{\prime}\right) * 100$

sendo que:

$T=$ conjunto de casos de teste original

$T_{S}=$ conjunto de casos teste de regressão selecionado.

$\mathbf{T}^{\prime}=$ conjunto de casos de teste que revelam um comportamento diferente entre $\mathrm{P}$ e $\mathrm{P}^{\prime}$ 
As seguintes etapas foram realizadas e são descritas a seguir:

Etapa 1 - Aplicação e Avaliação da Técnica baseada em Modificação

Etapa 2 - Aplicação e Avaliação da Técnica baseada em Mutação Seletiva

Dtapa 3 - Análise Comparativa dos Resultados obtidos com a Aplicação de ambas as Técnicas de Teste de Regressão com os resultados obtidos por Wong et al.(1997a, 1997b).

Etapa 4 - Avaliação das Técnicas de Teste de Regressão segundo o Framework de Rothermel e Harrold.

\subsubsection{Aplicação e Avaliação da Técnica baseada em Modificação}

Nesta Seção são descritos os passos realizados duraṇte a aplicação da técnica baseada em Modificação nos programas UNIX. Os seguintes passos foram realizados e são descritas a seguir:

$>$ Passo 1 - Preparação dos Dados dos Programas.

$>$ Passo 2 - Aplicação da Técnica baseada em Modificação nos programas UNIX.

Passo 3 - Análise dos Resultados.

\section{Passo l - Preparação dos Dados dos Programas}

Os conjuntos de casos de teste utilizados para os programa UNIX foram adquiridos do trabalho de Vincenzi (1998). pois para esses programas Vincenzi já havia definido os conjuntos de casos de teste adequados em relação ao critério Análise de Mutantes para cada um dos cinco programas. Para cada um dos programas, um conjunto de programas modificados foi utilizado. Esses programas contêm apenas uma modificação (defeito) em uma linha de código (Wong et al, 1997b). A Tabela 3.1 apresenta o número casos de teste e o número de defeitos disponíveis para cada programa. $O$ Apêndice $B$ apresenta os programas originais e suas modificações.

Utilizando-se um script escrito no $K$-shell do UNIX (Vergilio, 1997), uma Tabela de Eficácia contendo todos os defeitos que são revelados por cada um dos cinco conjuntos de casos de teste foi gerada. Por meio dessa Tabela de Eficácia foi possível estabelecer níveis de dificuldade de detecção de cada defeito. Foram definidos quatro niveis. Defeitos que estão no 
nível I são revelados pelo intervalo de [0-25\% dos casos de teste); defeitos que estão no nível II são revelados por [25-50\% dos casos de teste); defeitos que estão no nível III são revelados por [50-75\% dos casos de teste); e defeitos que estão no nível IV são revelados por [75-100\% dos casos de teste] (Wong et al., 1997b). Defeitos que estão no nível I são os mais dificeis de serem revelados, enquanto que aqueles que estão no nível IV são os mais făceis de serem revelados. A Tabela 3.2 mostra em que nível está cada um dos defeitos disponiveis para cada programa. Esses niveis de dificuldade estão relacionados sempre com o conjunto de casos de teste disponível.

Tabela 3.1 - Dados dos programas UNIX.

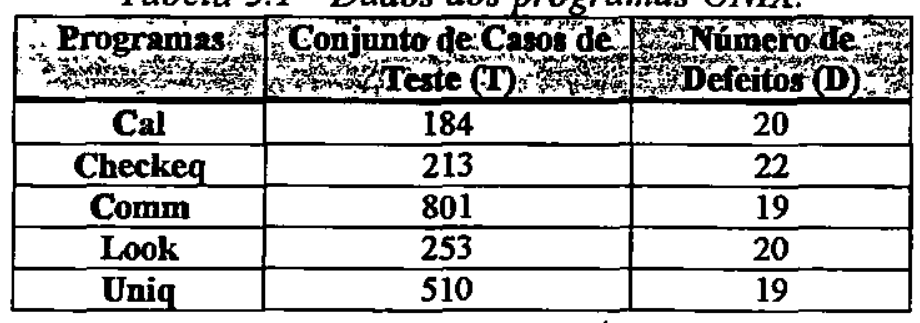

Tabela 3.2 - Categorização dos defeitos dos programas UNIX.

\begin{tabular}{|c|c|c|c|c|}
\hline Programas & \multicolumn{4}{|c|}{ 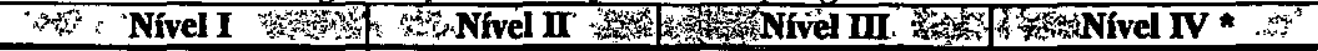 } \\
\hline Cal & $\begin{array}{l}\text { D2 D5 D6 D9 D18 } \\
\text { D20 }\end{array}$ & $\begin{array}{l}\text { D4 D11 D12 } \\
\text { D19 }\end{array}$ & $\begin{array}{c}\text { D3 D7 D8 D10 } \\
\text { D13 D14 D15 D16 } \\
\text { D17 }\end{array}$ & D1 \\
\hline Checkeq & D1 D6 D11 D13 D20 & $\overline{D 4}$ D19 D22 & $\begin{array}{l}\text { D2 D3 D8 D9 D12 } \\
\text { D14 D15 D16 D18 } \\
\text { D21 }\end{array}$ & D5 D7 D10 D17 \\
\hline Comm & $\begin{array}{c}\text { D2 D3 D4 D6 D7 D10 } \\
\text { D12 D13 D14 D16 D17 } \\
\text { D19 }\end{array}$ & D5 D8 D15 D18 & D9 D11 & D1 \\
\hline Look & $\begin{array}{c}\text { D1 D3 D4 D5 D6 D7 } \\
\text { D8 D9 D10 D11 D12 } \\
\text { D13 D14 D15 D18 D19 } \\
\text { D20 }\end{array}$ & D16 & D2 D17 & - \\
\hline Uniq & $\begin{array}{c}\text { D2 D3 D5 D6 D9 D10 } \\
\text { D11 D14 D15 D16 D18 } \\
\text { D19 }\end{array}$ & $\begin{array}{c}\text { D7 D8 D12 D13 } \\
\text { D17 }\end{array}$ & D1 D4 & - \\
\hline
\end{tabular}

* O símbolo "_" significa que não existem defeitos nesse nível.

Para cada um dos programas, a precisão e a eficácia do conjunto de casos de teste $\mathbf{T}$ foi calculada. A Tabela 3.3 mostra esses dados. Observando a tabela, nos programas Checkeq, Comm, Look e Uniq o conjunto $\mathrm{T}$ não é capaz de revelar todos os defeitos disponiveis. No entanto, para os programas Comm, Look e Uniq, os defeitos que não são revelados na verdade não são defeitos, são programas equivalentes ao programa original. Assim, qualquer que seja o conjunto de casos de teste, esse não será capaz de causar um comportamento diferente entre os programas originais e os modificados. Nesse caso, para essas modificações que não caracterizam 
defeitos, não foram selecionados conjuntos de casos de teste de regressão. Com relação à precisão, analisando-se a Tabela 3.3, pode-se notar que somente no caso do programa Checkeq todos os casos de teste disponíveis revelam pelo menos um defeito, pois a precisão foi de $100 \%$. O programa Uniq é aquele que possui, proporcionalmente, o menor número de casos de teste que revelam defeitos, ou seja, $21,858 \%$ dos casos de teste não revelam defeitos

Tabela 3.3-Precisão e eficácia do conjunto T dos programas UNIX.
\begin{tabular}{|c|c|c|}
\hline - & Programas \\
\hline Cal & 98,370 & 100 \\
\hline Checkeq & 100 & 95,455 \\
\hline Comm & 99,625 & 94,737 \\
\hline Look & 88,142 & 80,000 \\
\hline Uniq & 78,431 & 94,737 \\
\hline
\end{tabular}

Após a preparação dos dados programas, a técnica baseada em Modificação foi aplicada a cada programa.

\section{Passo 2 - Aplicação da Técnica baseada em Modificação nos Programas UNLX}

As seguintes tarefas foram executadas e são descritas a seguir:

Tarefa 1 - Definição do Conjunto $\mathrm{T}^{\prime}$ com relação a cada defeitos.

$>$ Tarefa 2 - Definição do Conjunto T" com relação aos programas priginais e seus defeitos.

$>$ Tarefa 3 - Seleção dos Conjuntos de Casos de Teste de Regressão para cada uma das Modificações.

\section{Tarefa 1 - Definiçäo do Conjunto $T$ 'com relação a cada defeitos}

O conjunto $T^{\prime}$ foi definido utilizando-se a Tabela de Eficácia gerada no passo anterior para cada um dos programas. Esse conjunto é composto por todos os casos de teste faultrevealing. A Tabela 3.4 mostra o tamanho de cada conjunto T' com relação a cada defeito. 
Tabela 3.4 - Conjunto T' para cada defeito.

\begin{tabular}{|c|c|c|c|c|c|}
\hline 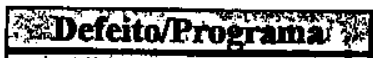 & 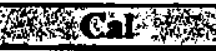 & Sheckeg & 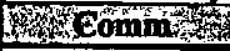 & 7 & H \\
\hline or & 138 & 33 & 794 & 1 & 260 \\
\hline W & 1 & 151 & 3 & 183 & 82 \\
\hline 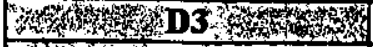 & 94 & 149 & $\mathbf{0}$ & 2 & 99 \\
\hline Fish & 89 & 101 & 26 & $\mathbf{0}$ & 338 \\
\hline H. & 5 & 202 & 347 & 23 & 89 \\
\hline Wor & 38 & $\mathbf{0}$ & 24 & 31 & 85 \\
\hline - & 127 & 202 & 12 & 34 & 131 \\
\hline 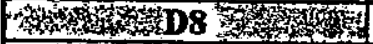 & 127 & 138 & 233 & 39 & 236 \\
\hline H4 & 41 & 137 & 462 & $\mathbf{0}$ & 65 \\
\hline 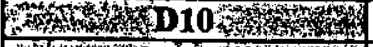 & 107 & 159 & 3 & $\begin{array}{ll}\mathbf{0} \\
\end{array}$ & 72 \\
\hline 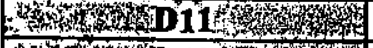 & 62 & 45 & 505 & 35 & $\mathbf{0}$ \\
\hline M & 90 & 153 & 125 & 3 & 142 \\
\hline 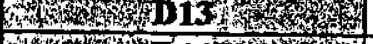 & 127 & 11 & 124 & 36 & 145 \\
\hline 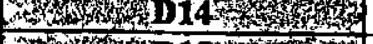 & 127 & 140 & 198 & 37 & 60 \\
\hline AN & 116 & 111 & 268 & 0 & 60 \\
\hline 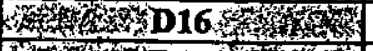 & 127 & 141 & 79 & 114 & 78 \\
\hline Do & 123 & 174 & 3 & 147 & 146 \\
\hline$\sqrt{2}$ & 29 & 147 & 248 & 2 & 21 \\
\hline 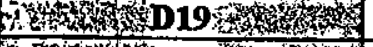 & 64 & 85 & 11 & 36 & 57 \\
\hline$y$, & 32 & 38 & - & 6 & 二 \\
\hline 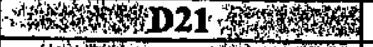 & 二 & 138 & - & 二 & - \\
\hline 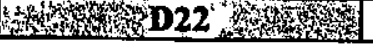 & $=$ & 83 & 二 & - & - \\
\hline
\end{tabular}

\section{Tarefa 2 - Definição dos Conjuntos $T$ " com relação aos programas originais e seus defeitos}

Os conjuntos T" são compostos pelos casos de teste modification-traversing. Esses conjuntos, evidentemente, contêm o conjunto $T$ '. Para a definição desses conjuntos, foi necessário estabelecer o trace de execução de cada um dos casos de teste disponíveis utilizandose a ferramenta PROTEUM/IM (Delamaro, 1997a).

Primeiramente, o código que sofre modificação em $\mathbf{P}$ para gerar $\mathrm{P}^{\prime}$ foi identificado através do grafo de fluxo de controle dos programas. Em seguida, uma sessão de teste foi gerada, para cada um dos programas, e todos os casos de teste disponiveis executados com os programas originais. Enquanto os casos de teste estavam sendo executados, um arquivo de trace para cada caso foi gerado. A partir desse arquivo, foi possível encontrar o caminho percorrido pelo caso de teste em $\mathbf{P}$ e verificar se esse caso de teste passava por alguma modificação. Se o caso de teste executasse a modificação, esse caso de teste era incluído no conjunto T" referente à modificação executada. Esses conjuntos, portanto, possuem todos os casos de teste modification-traversing com respeito a um determinado defeito. Considerando o conjunto original $\mathrm{T}$ de cada programa, todo conjunto possui pelo menos um caso de teste que executa pelo menos um defeito. Assim, a Tabela 3.5 mostra o número de casos de teste que estão nos conjuntos $T^{\text {” }}$ para cada um dos defeitos para cada programa. 
Tabela 3.5 - Conjunto T" para cada defeito.

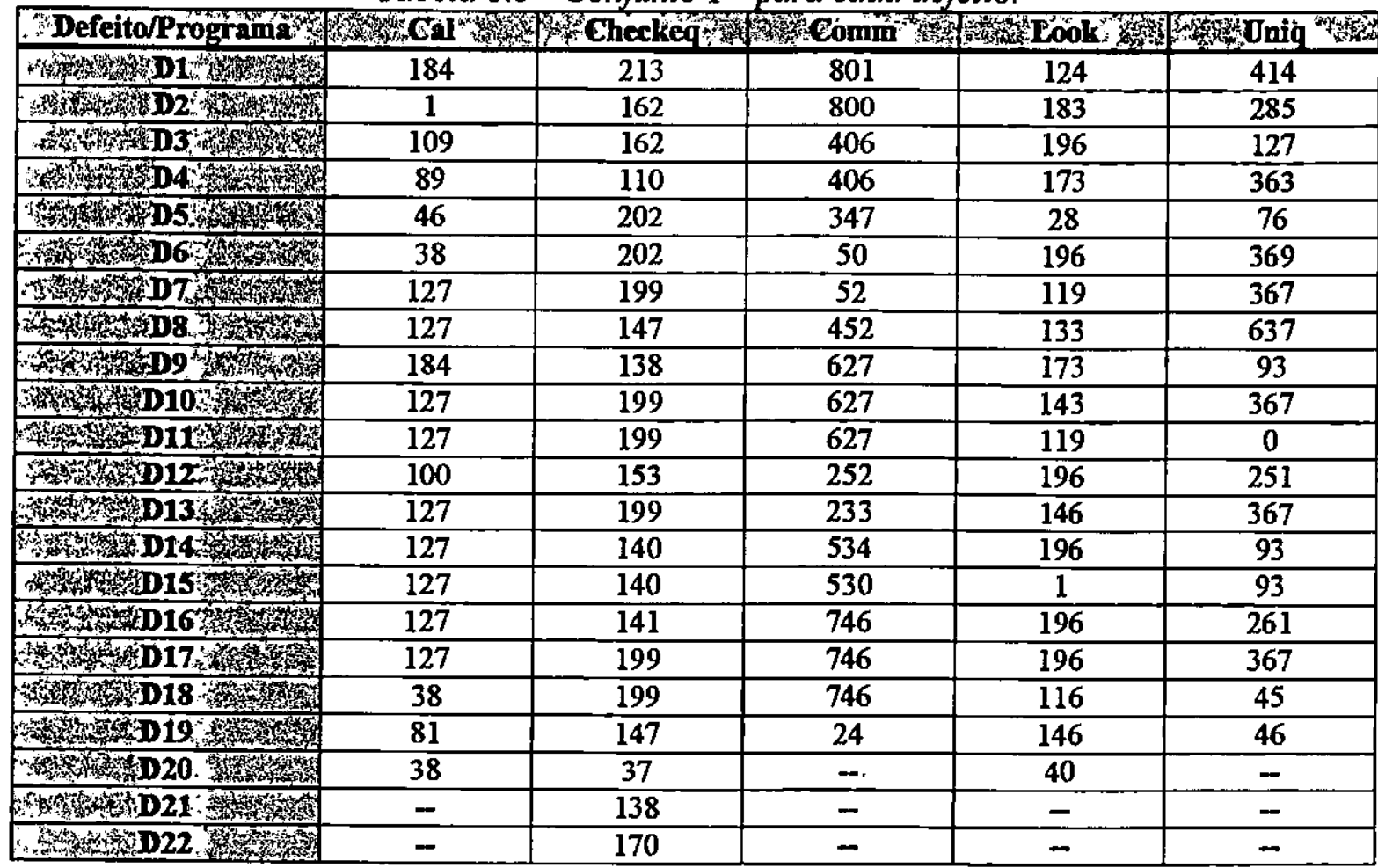

\section{Tarefa 3 - Seleção dos Conjuntos de Casos de Teste de Regressão para cada uma das Modificaçōes}

O principal objetivo da técnica é selecionar um subconjunto de casos de teste que passam por alguma modificação. Após a definição de cada um dos conjunto T", os mecanismos de minimização e priorização foram aplicados.

Selecionando os conjuntos de casos de teste de regressão utilizando o mecanismo de minimização

Primeiramente, uma sessão de teste foi criada para cada um dos conjuntos de casos de teste modification-traversing, T', selecionados para cada um dos defeitos. Para cada um dos programas, os mutantes foram gerados e executados com os conjuntos de casos de teste T". A Tabela 3.6 sintetiza número de mutantes gerados para cada um dos operadores de mutação essenciais. Em seguida, o módulo de minimização MINIMIZE foi aplicado a cada sessão de teste, gerando um conjunto de casos de teste de regressão minimizado, $\mathrm{T}^{\mathrm{M}}{ }_{\mathrm{M}}$, para cada um dos defeitos de cada programa, preservando o mesmo índice de adequação em relação ao conjuntos de 
operadores de mutação essenciais. A Tabela 3.7 apresenta o número de casos de teste em cada conjunto T"MDx.

Tabela 3.6-Número de mutantes gerados por operador de mutaçao essencial.

\begin{tabular}{|c|c|c|c|c|c|}
\hline Operadores/Programas & 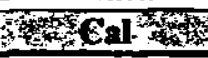 & \% Chedkeg & 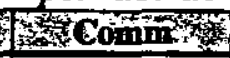 & 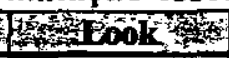 & Stonig \\
\hline SWDD & 2 & 2 & 4 & 3 & 7 \\
\hline SMTC & 8 & 4 & 5 & 7 & 9 \\
\hline SSDL & 101 & 70 & 103 & 82 & 90 \\
\hline OLBN & 12 & 33 & 9 & 12 & 15 \\
\hline ORRN & 110 & 135 & 115 & 60 & 95 \\
\hline VTWD & 124 & 130 & 50 & 74 & 44 \\
\hline VDTR & 186 & 195 & 75 & 111 & 66 \\
\hline Cccr & 939 & 340 & 164 & 125 & 108 \\
\hline Ccsr & 645 & 540 & 51 & 115 & 42 \\
\hline Total Gerado & 2127 & 1449 & 576 & 589 & 476 \\
\hline Total de Equivalentes & 204 & 126 & 102 & 118 & 43 \\
\hline
\end{tabular}

Tabela 3.7 - Número de casos de teste nos conjuntos minimizados T"

\begin{tabular}{|c|c|c|c|c|c|}
\hline Deféto/Programa & 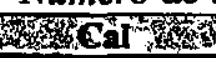 & 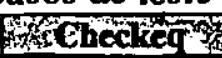 & 安 & 5 & Unig " \\
\hline 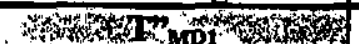 & 19 & 23 & 22 & 18 & 10 \\
\hline 45, & 1 & 9 & 26 & 14 & $\overline{4}$ \\
\hline 促 & 12 & 9 & 0 & 16 & $\overline{1}$ \\
\hline 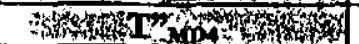 & 11 & 4 & 15 & $\overline{0}$ & 1 \\
\hline W & 10 & 22 & 12 & 8 & 1 \\
\hline (1) & 7 & $\begin{array}{ll}0 \\
0\end{array}$ & 4 & 16 & 2 \\
\hline H & 11 & 22 & 4 & 13 & 6 \\
\hline 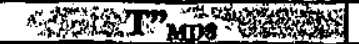 & 11 & 5 & 8 & 12 & 6 \\
\hline 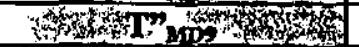 & 19 & 4 & 8 & 0 & 2 \\
\hline 7 th & $\overline{11}$ & 22 & 16 & $\overline{0}$ & 1 \\
\hline For & 11 & 23 & 16 & 12 & $\begin{array}{ll}0 & \\
\end{array}$ \\
\hline * & 10 & 6 & 6 & 16 & 4 \\
\hline 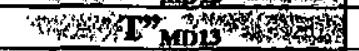 & 11 & 22 & 6 & 13 & 2 \\
\hline 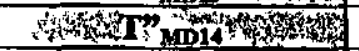 & 11 & 5 & 11 & 16 & 2 \\
\hline 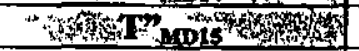 & 11 & 7 & 12 & $\overline{0}$ & 6 \\
\hline 3 & $\overline{11}$ & 6 & 22 & 16 & 2 \\
\hline W & $\overline{11}$ & 22 & 22 & 16 & 2 \\
\hline 20 & 8 & $\overline{22}$ & $\overline{6}$ & 18 & 1 \\
\hline 4 , & $\overline{8}$ & 5 & 6 & 13 & 1 \\
\hline rin & 7 & 5 & - & 8 & - \\
\hline W & Z & 4 & $=$ & - & 二 \\
\hline 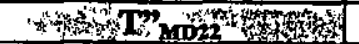 & - & 16 & - & - & - \\
\hline
\end{tabular}

Selecionando os conjuntos de casos de teste de regressão utilizando um mecanismo de priorização baseado na eficácia do caso de teste

Primeiramente, com o auxilio da Tabela de Eficácia, os conjuntos de casos de teste modification-traversing de cada um dos defeitos dos programas foram ordenados de acordo com a eficácia dos casos de teste: casos de teste que mais revelavam defeitos foram colocados no topo 
da lista de prioridades. Em seguida, esses casos de teste com maior prioridade foram sendo selecionados para os conjuntos de casos de teste priorizados T"PDx até que o defeito fosse revelado. Esse processo foi realizado para todos os defeitos de cada um dos programas UNIX. A Tabela 3.8 mostra o número de casos de teste selecionados pelo mecanismo de priorização para cada defeito de cada programa.

Em geral, para todos os programas o tamanho dos conjuntos priorizados é menor que o tamanho dos conjuntos minimizados. Esse fato evidencia a importância de se ter informações com relação aos casos de teste disponíveis de forma a minimizar a relação custo/beneficio.

Wong et al. utilizaram para a priorização informações sobre o incremento proporcionado na cobertura relativa a um determinado critério de teste. Essas informações podem ser consideradas para a definição de políticas de priorização. A decisão tomada neste trabalho de utilizar as informações sobre a eficácia dos casos de teste favorece a aplicação desta técnica do ponto de vista de custo/beneficio.

Tabela 3.8 - Número de casos de teste nos conjuntos priorizados $T^{\prime \prime}{ }_{P D x}$

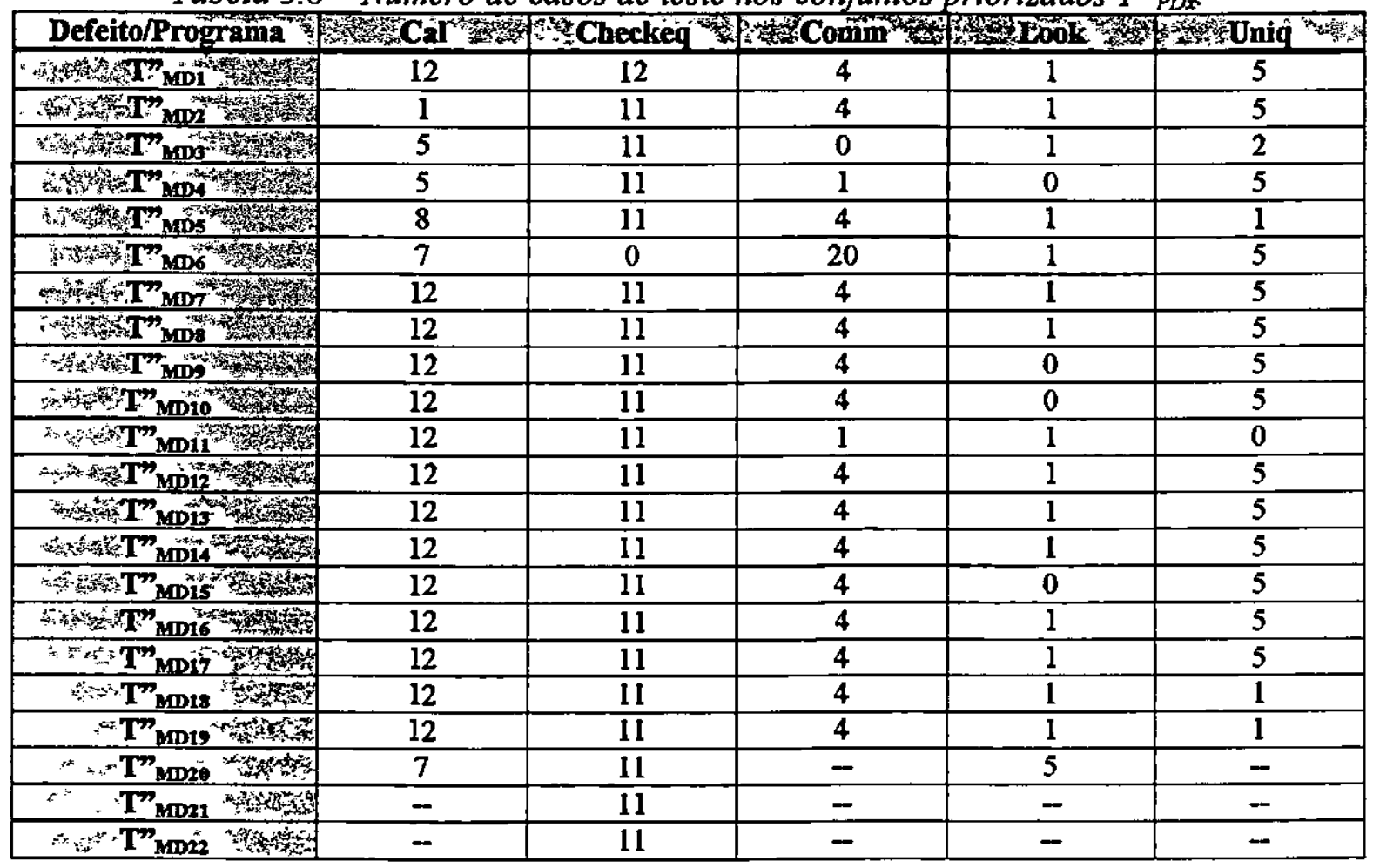

\section{Passo 3 - Análise dos Resultados}

As Tabelas 3.9 e 3.10 sintetizam os resultados dos experimentos apresentando a média dos valores correspondentes a cada um dos conjuntos minimizados e priorizados. Observa-se nas 
tabelas, que os valores de redução de tamanho e precisão foram um pouco melhores para os conjuntos priorizados. Já os valores de recall foram semelhantes para todos os conjuntos minimizados e priorizados, apresentando apenas uma pequena variação. A redução de eficácia para todos os conjuntos foi de $0 \%$. Os valores de precisão e recall são diretamente dependentes do número de casos de teste que revelam um defeito. Se existem poucos casos que revelam um determinado defeito, certamente os valores de recall serão altos e os valores de precisão serão baixos. No entanto, isso não significa que a técnica seja menos ou mais eficaz., ou seja, é uma medida relativa.

Nota-se que o uso da minimização baseada no conjunto de operadores essenciais de mutação produz resultados muitos bons com relação aos resultados produzidos com a priorização que utilizou informações sobre a eficácia dos casos de teste, que na realidade traz mais informações semânticas.

Tabela 3.9 - Média para os conjuntos minimizados $T "{ }_{M D x}$

\begin{tabular}{|c|c|c|c|c|}
\hline Programas & 3. & Sty & Row & 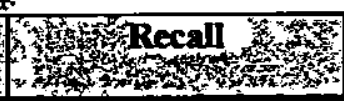 \\
\hline Cal & 83,926 & 100 & 59,815 & 16,293 \\
\hline Checkeg & 92,617 & 100 & 43,613 & 10,592 \\
\hline Comm & 95,608 & 100 & 48,636 & 9,737 \\
\hline Look & 88,558 & 100 & 73,315 & 27,243 \\
\hline Unig & 98,395 & 100 & 78,191 & 1,838 \\
\hline
\end{tabular}

Tabela 3.10-Média para os conjuntos priorizados $T_{P D x}$

\begin{tabular}{|c|c|c|c|c|}
\hline Programas & Weducho de & ofy & 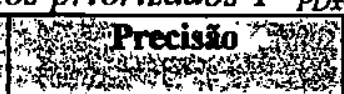 & (3) \\
\hline Cal & 83,954 & 100 & 90,625 & 18,159 \\
\hline Checkeg & 92,107 & 100 & 91,270 & 9,828 \\
\hline Comm & 95,655 & 100 & 72,778 & 13,740 \\
\hline Look & 98,346 & 100 & 93,750 & 21,435 \\
\hline Uniq & 97,787 & 100 & 88,889 & 3,723 \\
\hline
\end{tabular}

A seguir, a avaliação dos resultados com relação a cada programa é apresentada.

\section{Programa CAL}

As Tabelas 3.11 e 3.12 apresentam os resultados obtidos com cada um dos conjuntos minimizados e priorizados, respectivamente. No caso da eficácia de cada um dos conjuntos, tanto dos minimizados quanto dos priorizados, todos foram capazes de revelar os defeitos 
pretendidos, obtendo-se, assim, uma eficácia de $100 \%$ com relação a todos os defeitos, ou, redução de eficácia de $0 \%$ com relação ao conjunto $T^{\prime \prime}$.

Analisando as Tabelas 3.11 e 3.12, é possivel notar que os conjuntos priorizados obtiveram uma maior redução do tamanho sem afetar a eficácia do conjunto em revelar defeitos. Ainda, é importante ressaltar que com o mecanismo de priorização utilizado procura-se evitar que casos que não revelam defeitos sejam selecionados, enquanto que com o mecanismo de minimização utilizado esse controle é mais dificil de ser realizado, pois o algoritmo que o Módulo MINIMIZE implementa seleciona aqueles casos de teste que mais "matam" mutantes sem considerar sua eficácia em revelar defeitos. Nesse caso, três conjuntos minimizados

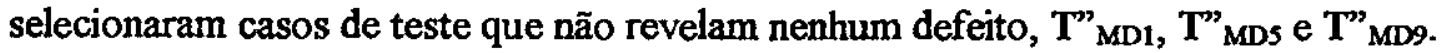

Tanto para os conjuntos minimizados quanto para os conjuntos priorizados, o recall foi pequeno e a precisão foi alta para a maioria dos defeitos. No entanto, para o defeito D2 tanto o recall quanto a precisão foram altos, sendo de $100 \%$. Isso ocorreu, pois no caso do defeito D2 somente um caso de teste do conjunto original é capaz de executá-lo e revelá-lo, e tanto o mecanismo de minimização quanto o de priorização foram capazes de selecionar apenas esse caso de teste. Esse fato também explica a redução de $0 \%$ no tamanho dos conjuntos minimizados e priorizados. No caso dos conjuntos priorizados, a maioria deles obteve $100 \%$ de precisão significando que todos os casos de teste selecionados pelo mecanismo de priorização são capazes de revelar um determinado defeito. Analisando ainda as Tabelas 3.11 e 3.12, é possível notar que muitas vezes o valor do recall e da redução de tamanho são complementares, atingindo $100 \%$. Esse fato ocorre quando o número de casos de teste que revelam e executam um defeito é o mesmo, como, por exemplo, para os defeitos D13 e D14. 
Tabela 3.11 - Cal: Resultados obtidos para cada um dos conjuntos minimizados $T^{\prime \prime}{ }_{M D x}$.

\begin{tabular}{|c|c|c|c|c|}
\hline Noférto & $\begin{array}{l}\text { Weducto de } \\
\text { Tamanho" }\end{array}$ & 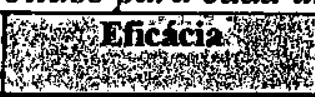 & Thy & Wy- \\
\hline$T_{M D 1}$ & 89,674 & 100 & 57,895 & 7,971 \\
\hline $\mathrm{T}_{\mathrm{MD2}}$ & 0 & 100 & 100 & 100 \\
\hline $\mathrm{T}_{\mathrm{MD} 3}$ & 88,991 & 100 & 75,000 & 9,574 \\
\hline $\mathrm{T}_{\mathrm{MDA}}$ & 87,640 & 100 & 100 & 12,360 \\
\hline$T_{M D S}$ & 78,261 & 100 & 20,000 & 40 \\
\hline $\mathrm{T}_{\mathrm{MD6}}$ & 81,579 & 100 & 100 & 18,421 \\
\hline $\mathrm{T}_{\mathrm{MD7}}$ & 91,339 & 100 & 100 & 8,661 \\
\hline $\mathrm{T}_{\mathrm{MDB}}$ & 91,339 & 100 & 100 & 8,661 \\
\hline$T_{M D 9}$ & 89,674 & 100 & 26,316 & 12,195 \\
\hline $\mathrm{T}_{\mathrm{MPlO}}$ & 91,339 & 100 & 72,727 & 7,477 \\
\hline$T_{M D 11}$ & 91,339 & 100 & 63,636 & 11,290 \\
\hline$T_{M 12}^{\prime \prime}$ & 90,000 & 100 & 70,000 & 7,778 \\
\hline$T^{\prime \prime}{ }_{M D 13}$ & 91,339 & 100 & 100 & 8,661 \\
\hline$T_{M D 14}$ & 91,339 & 100 & 100 & 8,661 \\
\hline $\mathrm{T}^{\prime \prime}{ }_{\mathrm{MDI} 5}$ & 91,339 & 100 & 81,818 & 7,759 \\
\hline$T^{\prime \prime}{ }_{M D 16}$ & 91,339 & 100 & 100 & 8,661 \\
\hline$T_{M D 17}$ & 91,339 & 100 & 100 & 8,943 \\
\hline T"'MD18 & 78,947 & 100 & 50,000 & 13,793 \\
\hline $\mathrm{T}_{\mathrm{MD19}}$ & 90,123 & 100 & 75,000 & 9,375 \\
\hline$T^{\prime \prime}{ }_{M D 20}$ & 81,579 & 100 & 71,429 & 15,625 \\
\hline
\end{tabular}

Tabela 3.12-Cal: Resultados obtidos para cada um dos conjuntos priorizados T'"

\begin{tabular}{|c|c|c|c|c|}
\hline Defeito & 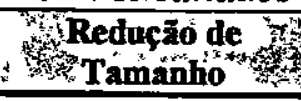 & W & W Trecis & 伤 \\
\hline$T^{\prime}{ }_{P D 1}$ & 93,478 & 100 & 41,667 & 3,623 \\
\hline$T^{\prime \prime}{ }_{P D 2}$ & 0 & 100 & 100 & 100 \\
\hline $\mathrm{T}_{\mathrm{PD3}}^{\prime \prime}$ & 95,413 & 100 & 100 & 5,319 \\
\hline$T^{\prime \prime}{ }^{\prime}$ & 94,382 & 100 & 100 & 5,618 \\
\hline$T^{\prime}$ PDS & 82,609 & 100 & 12,500 & 20,000 \\
\hline$T_{P D 6}$ & 81,579 & 100 & 100 & 18,421 \\
\hline $\mathrm{T}_{\mathrm{PD} 7}$ & 90,551 & 100 & 100 & 9,449 \\
\hline$T^{\prime}{ }_{\mathrm{PDB}}$ & 90,551 & 100 & 100 & 9,449 \\
\hline T"PD9 & 93,478 & 100 & 75,000 & 21,951 \\
\hline $\mathrm{T}_{\mathrm{PDIO}}$ & 90,551 & 100 & 100 & 11,215 \\
\hline$T_{\text {PDII }}^{\prime \prime}$ & 90,551 & 100 & 100 & 19,355 \\
\hline$T^{\prime \prime}{ }^{\prime} 12$ & 88,000 & 100 & 100 & 13,333 \\
\hline$T^{\prime \prime}{ }^{\prime}{ }^{2} 13$ & 90,551 & 100 & 100 & 9,449 \\
\hline$T^{\prime}$ PDI4 & 90,551 & 100 & 100 & 9,449 \\
\hline $\mathrm{T}_{\text {PDI } 5}$ & 90,551 & 100 & 100 & 10,345 \\
\hline $\mathrm{T}_{\mathrm{PD} 16}$ & 90,551 & 100 & 100 & 9,449 \\
\hline$T^{\prime \prime}{ }^{\prime \prime}{ }^{2} 17$ & 90,551 & 100 & 100 & 9,756 \\
\hline T"'PD18 & $68,42 I$ & 100 & 91,667 & $37,93 I$ \\
\hline $\mathrm{T}^{\prime \prime}{ }_{\text {PDI9 }}$ & 85,185 & 100 & 91,667 & 17,188 \\
\hline$T^{\prime \prime}{ }^{2 D 20}$ & 81,579 & 100 & 100 & 21,875 \\
\hline
\end{tabular}




\section{Programa CHECKEQ}

Os conjuntos minimizados obtiveram uma menor redução de tamanho do que os conjuntos priorizados. Conseqüentemente, os valores de recall para cada um dos defeitos foram maiores, pois mais casos de teste foram selecionados para o teste de regressão. $\mathrm{O}$ mesmo fato ocorre também com a precisão, que foi menor para a maioria dos defeitos para os conjuntos minimizados. Tanto para os conjuntos minimizados quanto para os conjuntos priorizados a redução de eficácia foi de $0 \%$ com relação aos conjunto T" ${ }^{\text {Dx. }}$. Assim, todos os conjuntos foram capazes de revelar os defeitos pretendidos com uma grande redução no número de casos de teste a serem reexecutados no teste de regressão.

Os resultados obtidos para cada um dos defeitos disponiveis para o programa Checkeq, para os conjuntos minimizados e priorizados, são mostrados nas Tabelas 3.13 e 3.14, respectivamente.

Tabela 3.13 - Checkeq: Resultados obtidos para cada um dos conjuntos minimizados $T^{\prime \prime}{ }_{M D x}$

\begin{tabular}{|c|c|c|c|c|}
\hline Defeito & Reducäo de & is & W & Fis \\
\hline$T^{\prime \prime}{ }_{M D I}$ & 89,202 & 100 & 39,130 & 27,273 \\
\hline $\mathrm{T}_{\mathrm{MD2}}$ & 94,444 & 100 & 88,889 & 5,298 \\
\hline $\mathrm{T}_{\mathrm{MD3}}^{\mathrm{NM}}$ & 94,444 & 100 & 100 & 6,040 \\
\hline$T_{M D 4}$ & 96,364 & 100 & 75 & 2,970 \\
\hline $\mathrm{T}_{\mathrm{MD5}}$ & 89,109 & 100 & 100 & 10,891 \\
\hline$T_{M D 6}$ & - & - & - & - \\
\hline $\mathrm{T}_{\mathrm{MD7}}$ & 88,945 & 100 & 100 & 10,891 \\
\hline $\mathrm{T}^{\prime \prime}{ }_{\mathrm{MDS}}$ & 96,599 & 100 & 100 & 3,623 \\
\hline $\mathrm{T}^{*}{ }_{\mathrm{MD} 9}$ & 97,101 & 100 & 75 & 2,190 \\
\hline $\mathrm{T}_{\mathrm{MD10}}^{n}$ & 88,945 & 100 & 45,455 & 6,289 \\
\hline$T_{M D 11}^{\prime \prime}$ & 88,945 & 100 & 27,273 & 13,333 \\
\hline $\mathrm{T}_{\mathrm{MD12} 2}^{m}$ & 96,078 & 100 & 100 & 3,922 \\
\hline $\mathrm{T}_{\mathrm{MD13}}^{\mathrm{m}}$ & 88,945 & 100 & 40,909 & 81,818 \\
\hline $\mathrm{T}_{\mathrm{MD14}}$ & 96,429 & 100 & 100 & 3,571 \\
\hline$T_{M D 15}$ & 95,000 & 100 & 100 & 6,306 \\
\hline $\mathrm{T}_{\mathrm{MDI6}}$ & 95,745 & 100 & 100 & 4,255 \\
\hline $\mathrm{T}_{\mathrm{MDI}} \mathrm{MDI0}$ & 88,945 & 100 & 72,727 & 9,195 \\
\hline $\mathrm{T}_{\mathrm{MD} 18}$ & 88,945 & 100 & 22,727 & 3,401 \\
\hline $\mathrm{T}_{M \mathrm{MD} 19}$ & 96,599 & 100 & 40 & 2,353 \\
\hline $\mathrm{T}_{\mathrm{MD20}}$ & 86,486 & 100 & 100 & 13,514 \\
\hline$T{ }_{M D 21}$ & 97,101 & 100 & 100 & 2,899 \\
\hline$T_{\mathrm{MP22}}$ & 90,588 & 100 & 12,500 & 2,410 \\
\hline
\end{tabular}


Tabela 3.14 - Checkeq: Resultados obtidos para cada um dos conjuntos priorizados $T_{P D x}{ }_{P D}$

\begin{tabular}{|c|c|c|c|c|}
\hline Wefeito & 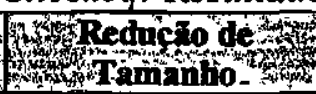 & 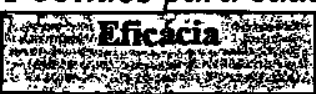 & 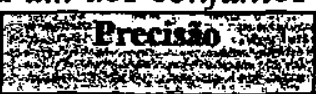 & (1) \\
\hline$T_{P D 1}$ & 94,366 & 100 & 8,333 & 3,030 \\
\hline$T_{P D 2}$ & 93,210 & 100 & 100 & 7,285 \\
\hline$T_{\text {PD3 }}$ & 93,210 & 100 & 100 & 7,383 \\
\hline$T^{\prime}{ }_{P D A}$ & 90 & 100 & 100 & 10,891 \\
\hline $\bar{T}_{\mathrm{PDS}}$ & 94,554 & 100 & 100 & 5,446 \\
\hline$T_{P D S}$ & $=$ & $=$ & $=$ & $=$ \\
\hline$T_{\mathrm{PD7} 7}$ & 94,472 & 100 & 100 & 5,446 \\
\hline$T^{\prime \prime}{ }_{\mathrm{PD} 8}$ & 92,517 & 100 & 100 & 7,971 \\
\hline$T_{P D 9}^{\prime}$ & 92,029 & 100 & 100 & 8,029 \\
\hline$T^{n}$ PDI0 & 94,472 & 100 & 100 & 6,918 \\
\hline$T^{n}$ PDI1 & 94,472 & 100 & 100 & 24,444 \\
\hline$T^{n}{ }_{\text {PD12 }}$ & 92,810 & 100 & 8,333 & 7,190 \\
\hline $\bar{T}_{\mathrm{PD} 13}$ & 94,366 & 100 & 100 & 9,091 \\
\hline$T_{\mathrm{PD} 14}$ & $92, \overline{143}$ & 100 & 100 & 7,857 \\
\hline$T_{\text {ppl5 }}$ & 92,143 & 100 & 100 & 9,910 \\
\hline$T_{p D 16}$ & 92,199 & 100 & 100 & 7,801 \\
\hline$T_{\mathrm{PD} 17}$ & 94,472 & 100 & 100 & 6,322 \\
\hline$T_{\mathrm{PD} 18}$ & 94,472 & 100 & 100 & 7,483 \\
\hline$T^{p}{ }_{p D 19}$ & 92,517 & 100 & 100 & 12,941 \\
\hline$T^{m}{ }_{p D 20}$ & 70,270 & 100 & 100 & 29,730 \\
\hline$T_{P D 21}^{m}$ & 92,029 & 100 & 100 & 7,971 \\
\hline$T_{\mathrm{PD22}}$ & 93,529 & 100 & 100 & 13,253 \\
\hline
\end{tabular}

\section{Programa COMM}

Para o programa Comm, os conjuntos priorizados também obtiveram uma maior redução de tamanho com relação aos conjuntos minimizados. Com relação aos valores de precisão e recall, os conjuntos minimizados obtiveram menores valores de precisão e maiores valores de recall do que os conjuntos priorizados. No entanto, a redução de eficácia para todos os conjuntos minimizados e priorizados foi de $0 \%$.

\section{Programa LOOK}

O programa Look foi o programa que obteve uma menor redução de tamanho dos conjuntos minimizados, pois 17, dos 20 defeitos disponiveis para o programa, estão no nível I de categorização de falhas, sendo assim, mais dificeis de serem revelados. Portanto, mais casos de teste devem ser reexecutados no teste de regressão. Com isso, os valores de recall foram maiores e os valores de precisão menores do que dos conjuntos priorizados. Já para os conjuntos priorizados, a redução de tamanho foi alta para todos os defeitos. A redução de eficácia para todos os conjuntos minimizados e priorizados foi de $0 \%$. 
Tabela 3.15-Comm: Resultados obtidos para cada um dos conjuntos minimizados $T^{\prime \prime}{ }_{M D x}$

\begin{tabular}{|c|c|c|c|c|}
\hline Wefeito & 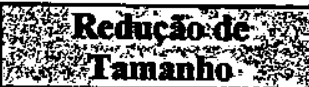 & (3) & 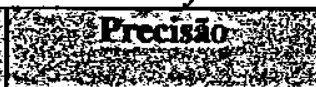 & W \\
\hline $\mathrm{T}_{\mathrm{MD1}}$ & 96,629 & 100 & 92,593 & 3,149 \\
\hline $\mathrm{T}_{\mathrm{MD2} 2}$ & 96,750 & 100 & 3,846 & 33,333 \\
\hline$T_{M D 3}$ & - & - & - & - \\
\hline $\mathrm{T}^{\prime \prime} \mathrm{MD}$ & 96,305 & 100 & 6,667 & 3,846 \\
\hline$T_{M D 5}$ & 96,542 & 100 & 91,667 & 3,170 \\
\hline $\mathrm{T}_{\mathrm{MD6}}$ & 92 & 100 & 50 & 8,333 \\
\hline$T^{\prime \prime}{ }^{2}$ ? & 92,308 & 100 & 25 & 8,333 \\
\hline$T_{M D 8}$ & 98,230 & 100 & 75 & 2,575 \\
\hline$T_{M D 9}^{\prime \prime}$ & 98,724 & 100 & 87,500 & 1,515 \\
\hline $\mathrm{T}_{\mathrm{MD} 10}$ & 97,448 & 100 & 6,250 & 33,333 \\
\hline$T^{7{ }^{3}}{ }_{\text {D11 }}$ & 97,448 & 100 & 93,750 & 2,970 \\
\hline $\mathrm{T}_{\mathrm{MD12}}$ & 97,619 & 100 & 16,667 & 0,800 \\
\hline $\mathrm{T}_{\mathrm{MD13}}$ & 97,425 & 100 & 33,333 & 1,613 \\
\hline$T_{M D 14}^{\prime \prime}$ & 97,940 & 100 & 54,545 & 3,030 \\
\hline $\mathrm{T}_{\mathrm{MD15}}$ & 97,736 & 100 & 91,667 & 4,104 \\
\hline $\mathrm{T}_{\mathrm{MD16}}$ & 97,051 & 100 & 9,091 & 2,532 \\
\hline $\mathrm{T}_{\mathrm{MD1}}$ & 97,051 & 100 & 4,545 & 33,333 \\
\hline $\mathrm{T}_{\mathrm{MD18}}$ & 98,739 & 100 & 83,333 & 2,016 \\
\hline T"MD19 & 75 & 100 & 50 & 27,273 \\
\hline
\end{tabular}

Tabela 3.16-Comm: Resultados obtidos para cada um dos conjuntos priorizados T" ${ }_{P D x}$

\begin{tabular}{|c|c|c|c|c|}
\hline Defeito & 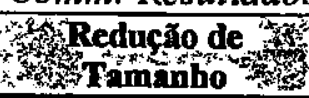 & 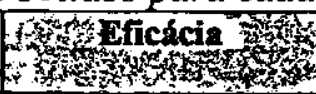 & 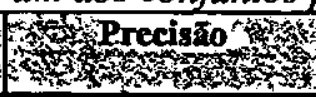 & W \\
\hline $\mathrm{T}_{\mathrm{PD1}}$ & $9 \overline{99,501}$ & 100 & 100 & 0,504 \\
\hline $\mathrm{T}^{10}{ }_{\mathrm{PD2}}$ & 99,375 & 100 & 20 & 33,333 \\
\hline $\mathrm{T}_{\mathrm{PD} 3}$ & - & - & - & - \\
\hline$T_{P D 4}$ & 99,507 & 100 & 50 & 3,846 \\
\hline T"PDS & 98,847 & 100 & 100 & 1,153 \\
\hline $\mathrm{T}_{\mathrm{PD} 6}$ & 60 & 100 & 90 & 75 \\
\hline$\widehat{T}{ }^{\prime}{ }^{2} 7$ & 90,385 & 100 & 40 & 16,667 \\
\hline $\mathrm{T}^{\prime \prime}{ }_{\mathrm{PD} 8}$ & 99,115 & 100 & 100 & 1,717 \\
\hline T"PD9 & 99,362 & 100 & 100 & 0,866 \\
\hline $\mathrm{T}_{\mathrm{PD} 10}$ & $9 \overline{99,203}$ & 100 & 20 & 33,333 \\
\hline$T^{\prime \prime}$ PDI1 & 99,362 & 100 & 100 & 0,792 \\
\hline $\mathrm{T}_{\mathrm{PD} 12}$ & 99,206 & 100 & 50 & 0,800 \\
\hline $\mathrm{T}_{\mathrm{PD} 13}$ & 98,283 & 100 & 100 & 3,226 \\
\hline $\mathrm{T}_{\mathrm{PDI}}$ & 99,251 & 100 & 100 & 2,020 \\
\hline T"PD15 & 99,245 & 100 & 100 & 1,493 \\
\hline$T^{3}{ }^{\prime}$ & 99,330 & 100 & 20 & 1,266 \\
\hline$\overline{T " P D 17}$ & 99,330 & 100 & 20 & 33,333 \\
\hline$T^{2}{ }^{2}$ & 99,160 & 100 & 100 & 1,613 \\
\hline 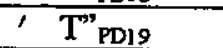 & 83,333 & 100 & 100 & 36,364 \\
\hline
\end{tabular}


Tabela 3.17-Look: Resultados obtidos para cada um dos conjuntos minimizados $T^{\prime \prime}{ }_{M D x}$ -

\begin{tabular}{|c|c|c|c|c|}
\hline 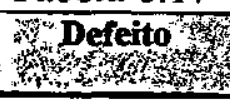 & 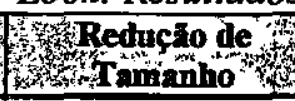 & H.t. & 13) & 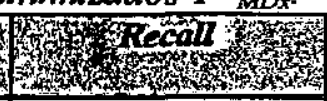 \\
\hline$T_{M D I}^{\prime \prime}$ & 85,484 & 100 & 5,556 & 100 \\
\hline $\mathrm{T}_{\mathrm{MD2}}$ & 92,350 & 100 & 100 & 7,650 \\
\hline $\mathrm{T}_{\mathrm{MD3}}$ & 91,837 & 100 & 6,250 & 50 \\
\hline$T_{M D 4}^{m}$ & - & - & - & - \\
\hline $\mathrm{T}_{\mathrm{MDS}}$ & 71,429 & 100 & 37,500 & 13,043 \\
\hline $\mathrm{T}^{\prime \prime} \mathrm{MDO}$ & 91,837 & 100 & 31,250 & 16,129 \\
\hline $\mathrm{T}_{\mathrm{MD} 7}$ & 89,076 & 100 & 53,846 & 20,588 \\
\hline$T_{M D 8}$ & 90,977 & 100 & 58,333 & 17,949 \\
\hline$T_{\mathrm{MD} 9}^{\prime}$ & - & $=$ & - & $=$ \\
\hline$T_{M D 10}$ & 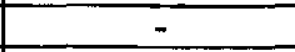 & - & - & $=$ \\
\hline $\mathrm{T}_{\mathrm{MD11}}$ & 89,916 & 100 & 75 & 25,714 \\
\hline$T_{\mathrm{MDI} 2}$ & 91,837 & 100 & 6,250 & 33,333 \\
\hline$T_{M D 13}$ & 81,096 & 100 & 61,538 & 22,222 \\
\hline $\bar{T}_{\mathrm{MDI4}}$ & 91,837 & 100 & 50 & 21,622 \\
\hline$\overline{\mathrm{T}_{\mathrm{MDIS}}}$ & - & - & - & - \\
\hline $\bar{T}_{\mathrm{MD16}}$ & 91,837 & 100 & $\overline{75}$ & 10,526 \\
\hline$T_{\mathrm{MDI7}}$ & 91,837 & 100 & 50 & 5,442 \\
\hline$T_{M D 18}$ & 84,483 & 100 & 5,556 & 50 \\
\hline $\mathrm{T}_{\mathrm{MDI}}^{\mathrm{m}}$ & 91,096 & 100 & 69,231 & 25 \\
\hline$T^{\prime \prime}{ }_{M 020}$ & 80 & 100 & 12,500 & 16,667 \\
\hline
\end{tabular}

Tabela 3.18 - Look: Resultados obtidos para cada um dos conjuntos priorizados $T_{P D x}$.

\begin{tabular}{|c|c|c|c|c|}
\hline Defeito & $\begin{array}{l}\text { Reducra de } \\
\text { Tánanho }\end{array}$ & (5) & 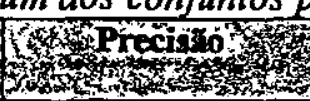 & 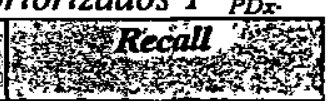 \\
\hline$T^{\prime}{ }_{P D I}$ & 98,387 & 100 & 50 & 100 \\
\hline$T_{\mathrm{PD} 2}$ & 99,454 & 100 & 100 & 0,546 \\
\hline $\bar{T}_{\mathrm{PD} 3}$ & 98,980 & 100 & 50 & 50 \\
\hline$T_{P D 4}^{\prime \prime}$ & - & - & - & - \\
\hline$T_{P D S}^{\prime \prime}$ & 99,429 & 100 & 100 & 4,348 \\
\hline$T_{P D G}$ & 99,490 & 100 & 100 & 3,226 \\
\hline $\mathrm{T}_{\mathrm{PD} 7}^{\prime \prime}$ & 99,160 & 100 & 100 & 2,941 \\
\hline$T_{\text {PD8 }}^{\prime \prime}$ & 99,248 & 100 & 100 & 2,564 \\
\hline$T_{\mathrm{PD} 9}$ & - & - & - & - \\
\hline T"PDIO & - & - & - & - \\
\hline $\bar{T}^{*}{ }_{\mathrm{PD1} 1}$ & 99,160 & 100 & 100 & 2,857 \\
\hline$\overline{T_{\mathrm{PD1} 12}}$ & 99,490 & 100 & 100 & 33,333 \\
\hline$T{ }^{P D 13}$ & 99,315 & 100 & 100 & 2,778 \\
\hline $\bar{T}_{\mathrm{PD} 14}$ & 99,490 & 100 & 100 & 2,703 \\
\hline$T_{\mathrm{PDIS}}$ & - & - & - & - \\
\hline$T_{\mathrm{PD16}}$ & 99,490 & 100 & $10 \overline{0}$ & 0,877 \\
\hline$\Gamma^{\prime \prime}{ }^{\prime} 17$ & 99,490 & 100 & 100 & 0,680 \\
\hline$T_{\mathrm{PD} 18}^{\prime \prime}$ & 99,138 & 100 & 100 & 50 \\
\hline T"PDI9 & 99,315 & 100 & 100 & 2,778 \\
\hline$\overline{\Gamma^{\prime}{ }_{\mathrm{PD} 20}}$ & 87,500 & 100 & 100 & 83,333 \\
\hline
\end{tabular}

\section{Programa UNIQ}

Tanto para os conjuntos minimizados quanto para os conjuntos priorizados, o programa Uniq obteve grandes reduções de tamanho e baixos valores para o recall. Essa alta redução de tamanho não afetou a eficácia dos conjuntos em revelar os defeitos, sendo que para todos os 
conjuntos minimizados e priorizados a eficácia foi de $100 \%$. As Tabelas 3.19 e 3.20 mostram os resultados obtidos com os conjuntos minimizados e priorizados com relação a cada um dos defeitos, respectivamente.

Tabela 3.19 - Uniq: Resultados obtidos para cada um dos conjuntos minimizados $T{ }_{M D_{x}}$

\begin{tabular}{|c|c|c|c|c|}
\hline 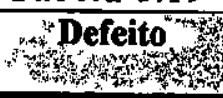 & 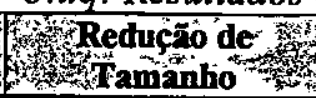 & F & 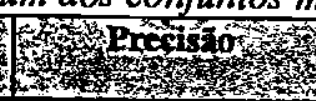 & Whecall \\
\hline $\mathrm{T}_{\mathrm{MDI}}$ & 97,585 & 100 & 60 & 2,308 \\
\hline$T_{M D 2}^{\prime \prime}$ & 98,596 & 100 & 0 & 1,220 \\
\hline $\mathrm{T}_{M{ }_{M D 3}}$ & 99,213 & 100 & 100 & 1,010 \\
\hline T"MD4 & 99,725 & 100 & 100 & 0,296 \\
\hline$T_{M M S}$ & 98,684 & 100 & 100 & 1,124 \\
\hline $\mathrm{T}_{\text {MD6 }}$ & 99,458 & 100 & 50 & 1,176 \\
\hline$T_{M D 7}$ & 98,365 & 100 & 16,667 & 0,763 \\
\hline$T_{M D 8}$ & 98,365 & 100 & 33,333 & 0,847 \\
\hline $\mathrm{T}_{\mathrm{MD} 9}^{\mathrm{M}}$ & 97,849 & 100 & 100 & 3,077 \\
\hline $\mathrm{T}_{\mathrm{MD} 10}$ & 99,728 & 100 & 100 & 1,389 \\
\hline $\mathrm{T}_{M D 11}$ & - & - & - & $=$ \\
\hline $\mathrm{T}_{\mathrm{MD12}}^{\prime \prime}$ & 98,406 & 100 & 25 & 0,704 \\
\hline $\mathrm{T}_{\mathrm{MD13}}$ & 99,455 & 100 & 50 & 0,690 \\
\hline$T_{M P 14}^{\prime \prime}$ & 97,849 & 100 & 100 & 3,333 \\
\hline$T_{M D 15}$ & 93,548 & 100 & 66,667 & 6,667 \\
\hline $\mathrm{T}_{\mathrm{MDI6}}$ & 99,234 & 100 & 50 & 1,282 \\
\hline$T_{M D 17}^{\prime \prime}$ & 99,455 & 100 & 50 & 0,685 \\
\hline T"'MD18 & 97,778 & 100 & 100 & 4,762 \\
\hline$T_{M D 19}$ & 97,826 & 100 & 100 & 1,754 \\
\hline
\end{tabular}

Tabela 3.20 - Uniq: Resultados obtidos para cada um dos conjuntos priorizados $T^{\prime \prime}{ }_{P D x}$

\begin{tabular}{|c|c|c|c|c|}
\hline Defeito & 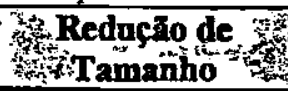 & 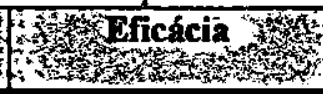 & W & W Recall \\
\hline $\mathrm{T}_{\mathrm{PD} 1}$ & 98,792 & 100 & 100 & 1,923 \\
\hline $\mathrm{T}_{\mathrm{PD2}}^{\prime \prime}$ & 98,246 & 100 & 60 & 3,659 \\
\hline $\mathrm{T}_{\mathrm{PD3}}^{\prime \prime}$ & 98,425 & 100 & 100 & 2,020 \\
\hline $\mathrm{T}_{\mathrm{PD4}}$ & 98,623 & 100 & 100 & 1,479 \\
\hline $\mathrm{T}_{\mathrm{PDS}}{ }_{\mathrm{P}}$ & 98,684 & 100 & 100 & 1,124 \\
\hline $\mathrm{T}_{\mathrm{PD6}}$ & 98,645 & 100 & 40 & 2,353 \\
\hline $\mathrm{T}_{\mathrm{PD} 7}$ & 98,638 & 100 & 80 & 3,053 \\
\hline $\mathrm{T}_{\mathrm{PD8}}$ & 98,638 & 100 & 100 & 2,119 \\
\hline $\mathrm{T}_{\mathrm{PD} 9}$ & 94,624 & 100 & 100 & 7,692 \\
\hline $\mathrm{T}_{\mathrm{PD} 10}$ & 98,638 & 100 & 60 & 4,167 \\
\hline$T_{\text {PDII }}^{\prime \prime}$ & - & - & - & - \\
\hline $\mathrm{T}_{\mathrm{PD12}}$ & 98,008 & 100 & 100 & 3,521 \\
\hline T"PDI3 & 98,638 & 100 & 100 & 3,448 \\
\hline$T_{\text {PDI4 }}^{\prime \prime}$ & 94,624 & 100 & 100 & 8,333 \\
\hline $\mathrm{T}^{\prime} \mathrm{PDI} 5$ & 94,624 & 100 & 100 & 8,333 \\
\hline$T_{\mathrm{PDI} 6}$ & 98,084 & 100 & 60 & 3,846 \\
\hline $\mathrm{T}_{\mathrm{PD} 17}$ & 98,638 & 100 & 100 & 3,425 \\
\hline$T_{\text {PD18 }}^{\prime \prime}$ & 97,778 & 100 & 100 & 4,762 \\
\hline$T^{m p D l 9}$ & 97,826 & 100 & 100 & 1,754 \\
\hline
\end{tabular}




\subsubsection{Aplicaçăo e Avaliação da Técnica baseada em Mutaçăo Seletiva}

Nesta Seção são descritos os passos realizados durante a aplicação da técnica baseada em Mutação Seletiva no programa SPACE. Relembrando, Wong et al. utilizaram-se dos programas UNIX para aplicar a técnica baseada em Mutação Seletiva.

Os seguintes passos foram realizados e são descritos a seguir:

Passo 1 - Preparação dos dados do Programa.

Passo 2 - Definição dos Operadores de Mutação.

Passo 3 - Aplicação da Técnica baseada em Mutação Seletiva no programa SPACE.

Passo 4 - Análise dos Resultados obtidos.

\section{Passo 1 - Preparação dos dados do Programa}

Como já mencionado, o programa utilizado para esses experimentos foi o programa SPACE. No entanto, algumas modificações no código fonte do programa foram realizadas para que o mesmo se adequasse à ferramenta PROTEUM1.4.1. Essas modificações foram realizadas na passagem de parâmetro das funções devido a algumas limitações da ferramenta em lidar com o tipo de passagem de parâmetro que estava sendo utilizada no programa. Foram modificadas todas as funções que compõem o programa. A Tabela 3.21 mostra os dados relacionados ao programa SPACE.

Tabela 3.21 - Dados do Programa SPACE.

\begin{tabular}{|c|c|c|}
\hline SPrograma & $\begin{array}{l}\text { Conjunito de Colos de } \\
\text { (T) }\end{array}$ & 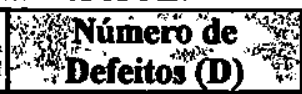 \\
\hline Space & 1000 & 10 \\
\hline
\end{tabular}

Assim como realizado com os programas UNIX, para os defeitos do programa SPACE, por meio da Tabela de Eficácia, estabeleceu-se os niveis de categorização para os dez defeitos disponíveis para o programa. Os seguintes níveis de categorização foram definidos de acordo com o conjunto de casos de teste disponível: Nível I - [0-250); Nível II - [250-500); Nível III [500-750); e Nível IV - [750-1000]. A Tabela 3.22 mostra em qual nível um determinado defeito está. 
Para o programa SPACE as versões modificadas foram geradas a partir da ativação dos defeitos no programa original. Ativado o defeito, um programa executável da versão modificada foi gerado e utilizado para o experimento.

Tabela 3.22 - Categorização dos Defeitos do Programa SPACE.

\begin{tabular}{|c|c|c|c|c|}
\hline Programa & Whing & 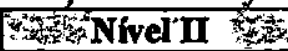 & Privel III & 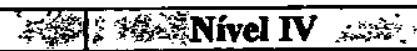 \\
\hline Space & $\begin{array}{c}\text { D1 D2 D3 D4 D5 D6 } \\
\text { D7 D8 D9 }\end{array}$ & D10 & 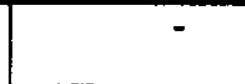 & - \\
\hline
\end{tabular}

* O símbolo "-" significa que não existem defeitos nesse nível.

Como se pode notar, a maioria das defeitos disponíveis são dificeis de serem revelados, 90\% dos defeitos estão no nível I. Analisando a Tabela de Eficácia gerada, 494 casos de teste, dos 1000 disponíveis, são capazes de revelar defeitos. Assim, a precisão do conjunto de casos de teste original é de 49,4\%. Entretanto, dos dez defeitos disponíveis, apenas o defeito D8 não foi revelado pelo conjunto de casos de teste original. Como esse defeito não é revelado pelo conjunto $\mathrm{T}$, a eficácia desse conjunto é de $90 \%$. A Tabela 3.23 mostra o número de casos de teste que revelam uma determinado defeito.

Tabela 3.23 - Número de Casos de Teste que revelam os Defeitos do Programa SPACE.

\begin{tabular}{|c|c|c|c|c|c|c|c|c|c|}
\hline D1 & \%D2 & gD3 & \$D4 & 政D5 & 1506 & : & D8 & - & \\
\hline 18 & 18 & 106 & 3 & 3 & 19 & 19 & - & 29 & 408 \\
\hline
\end{tabular}

\section{Passo 2 - Definição dos Operadores de Mutação}

Os operadores de mutação utilizados nos experimentos são os operadores essenciais definidos por Barbosa (1998). Barbosa definiu um conjunto de 9 operadores de mutação, a partir dos 71 operadores de mutação definidos na ferramenta PROTEUM.

Segundo Barbosa, o conjunto de operadores de mutação essenciais inclui alguns dos operadores de mutação utilizados por Wong et $a l^{4}$. Os operadores incluídos pelos operadores essenciais são: CRCR, OLLN e OLNG. A Figura 3.1 mostra a relação entre ambos os conjuntos de operadores de mutação e a Tabela 3.24 mostra os operadores de mutação essenciais que estão sendo utilizados e o número de mutantes gerados por cada um deles para o Programa SPACE. A Tabela 3.25 mostra o número de mutantes que são gerados pelo operadores de

\footnotetext{
${ }^{4}$ Um operador A inclui um outro opcrador B quando um conjunto de casos de teste adequado ao operador A é também adequado ao operador $B$.
} 
mutação utilizados por Wong et al. A descrição de cada um dos operadores de mutação das Tabelas 3.24 e 3.25 está no Apêndice A.

Tabela 3.24 - Conjunto de Operadores Essenciais e os Mutantes Gerados para cada Operador.

\begin{tabular}{|c|c|}
\hline Operadores de & Wutantes feredos \\
\hline SWDD & 37 \\
\hline SMTC & 53 \\
\hline SSDL & 3848 \\
\hline$\overline{\text { OLBN }}$ & 153 \\
\hline ORRN & 2765 \\
\hline VTWD & 2822 \\
\hline VDTR & 4233 \\
\hline $\mathrm{Cccr}$ & 37504 \\
\hline Cesr & 4077 \\
\hline Total & 55.492 \\
\hline
\end{tabular}

Tabela 3.25-Total de Mutantes gerados para o Conjunto de Operadores de Mutação definidos por

\begin{tabular}{|c|c|}
\hline \multicolumn{2}{|c|}{ Wong. } \\
\hline 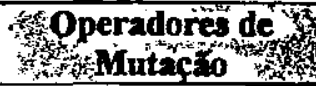 & $y_{3}^{3}$ \\
\hline VDTR & 4233 \\
\hline VTWD & 2822 \\
\hline SSDL & 3848 \\
\hline SMVB & 96 \\
\hline SBRn & 0 \\
\hline SCRn & 0 \\
\hline Vprr & 4210 \\
\hline Vtrr & 0 \\
\hline CRCR & 5655 \\
\hline$\overline{\mathrm{OLLN}}$ & 51 \\
\hline$\overline{\text { OLNG }}$ & 153 \\
\hline ORRN & 2765 \\
\hline Total & 23.833 \\
\hline
\end{tabular}

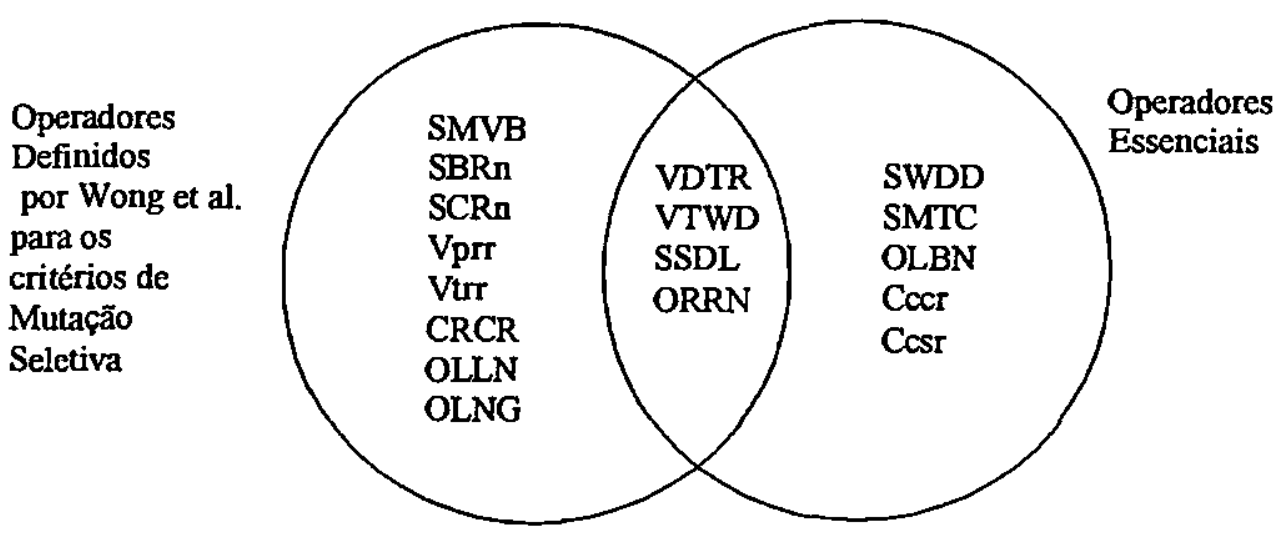

Figura 3.l - Relação entre os Conjuntos de Operadores de Mutação. 
Passo 3 - Aplicação da Técnica baseada em Mutação Seletiva no Programa SPACE

Seguindo os passos realizados por Wong (1997a), vide Figura 2.3, e utilizando-se a ferramenta PROTEUM, uma sessão de teste foi realizada com o programa SPACE. Após a geração dos mutantes, cada caso de teste t do conjunto $T$ original foi executado um a um. Se esse caso de teste $t$ melhorasse o escore de mutação, então esse era incluído no conjunto T’. Esse procedimento prosseguiu até que todos os casos de teste fossem executados. Ao final do processo, o conjunto T' $\subset$ T AM_adequado foi selecionado.

Ao final do processo de execução dos mutantes, nem todos os mutantes foram distinguidos pelo conjunto de casos de teste disponivel. Devido à complexidade do programa SPACE e do alto custo em analisar esses mutantes, todos foram considerados e marcados como equivalentes. Essa, certamente, não é uma abordagem precisa para determinação dos mutantes equivalentes, porém é conservadora. Pode ocorrer que mutantes que não são equivalentes sejam considerados como tal. Como conseqüência, durante a construção do conjunto AM_adequado, pode-se deixar de selecionar um caso de teste que distinguiria aquele mutante. Isso, por sua vez, só pode fazer com o que o conjunto AM_adequado seja menos eficaz em revelar um defeito, mas nunca mais eficaz. Dessa forma, as medidas de eficácia são pessimistas (Delamaro, 1997a).

Ao final do processo descrito acima, o conjunto T' definido para o programa SPACE com relaçăo aos operadores essenciais de mutação possui 229 casos de teste. No entanto, como se pode notar pela Tabela 3.23, nenhum caso de teste do conjunto $T$ original é capaz de revelar o defeito D8. Assim, um novo caso de teste foi selecionado, de um outro conjunto de casos de teste para o programa SPACE, e inserido em T'.

\section{Passo 4 - Análise dos Resultados obtidos}

A Tabela 3.26 mostra os resultados obtidos com os 229 casos de teste selecionados pelo processo descrito acima com relação ao conjunto de casos de teste original $\mathrm{T}$. A redução de eficácia foi de $0 \%$, pois o programa original não revela o defeito D8, como já mencionado.

A Tabela 3.27 mostra os resultados da precisão e do recall do conjunto T' com relação a cada defeito. Os valores de recall foram relativamente baixos com relação aos defeitos, média de $36,281 \%$. O mesmo ocorre com a precisão de T' com relação a cada um dos defeitos, com média 
de 8,626 . Nota-se pela Tabela 3.23 que os defeitos que possuem um número maior de casos de teste que o revelam, são aqueles com os maiores valores de precisão. Dos 229 casos de teste que foram selecionados, 95 não revelam defeito. Sendo assim, precisão do subconjunto de teste de regressão é relativamente baixa, 58,515\%.

Tabela 3.26 - Redução de Tamanho, Eficácia, Precisão e Recall do conjunto T' selecionado.

\begin{tabular}{|c|c|c|c|}
\hline $\begin{array}{l}\text { Reduçāo de } \\
\text { Tamanho }\end{array}$ & Reducão de & if:Precisio & Recall \\
\hline 77,100 & $\overline{0}$ & 58,515 & 27,126 \\
\hline
\end{tabular}

Tabela 3.27- Precisão e Recall do conjunto $T^{\prime}$ com relação a cada defeito.

\begin{tabular}{|c|c|c|}
\hline 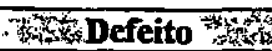 & 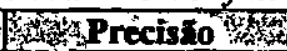 & 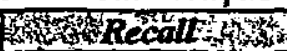 \\
\hline D1 & 3,057 & 38,889 \\
\hline D2 & 4,637 & 55,556 \\
\hline D3 & 11,790 & 25,472 \\
\hline$\overline{D 4}$ & 0,437 & 33,333 \\
\hline D5 & 0,437 & 33,333 \\
\hline D6 & 2,183 & 26,316 \\
\hline D7 & 2,183 & 26,316 \\
\hline$\overline{\mathrm{D} 8}$ & - & - \\
\hline$\overline{D 9}$ & 7,860 & 62,069 \\
\hline D10 & 44,978 & 25,245 \\
\hline
\end{tabular}

\subsubsection{Análise Comparativa dos Resultados obtidos com a aplicação de ambas as Técnicas de Teste de Regressão com os resultados obtidos por Wong et al. (1997a, 1997b)}

Para o experimento conduzido por Wong et al. (1997a), descrito no capítulo anterior, utilizando a técnica baseada em Modificação no programa SPACE, em média, os conjuntos baseados em modificação continham 80,3 casos de teste modification-traversing, sendo o menor com quatro casos de teste e o maior com 470 casos de teste. Os conjuntos minimizados, em média, continham 9,9 casos de teste, e os conjuntos priorizados continham $3,4,6,8$ e 9,9 casos de teste. Como se pode notar, os conjuntos priorizados obtiveram uma maior redução do número de casos de teste a serem reexecutados. Para os conjuntos minimizados a média de redução de tamanho, com relação aos conjuntos T", foi de 70,917\% e para os conjuntos priorizados foi de $88,552 \%, 77,103 \%$ e $70,917 \%$. Já os valores de precisão e recall para os dez conjuntos minimizados foram de $71,763 \%$ e $35,9 \%$, respectivamente, em média. Para os conjuntos priorizados os valores de precisão foram $69,001 \%, 68,083 \%$ e $70,254 \%$ e os valores de recall foram $12,038 \%, 22,561 \%$ e $34,742 \%$. Todos os conjuntos minimizados e priorizados obtiveram uma eficácia de $100 \%$ para todos os defeitos. 
Para os experimentos conduzidos durante este trabalho, uma síntese dos resultados estão na Tabela 3.28. Em média, a técnica foi capaz de reduzir bastante o número de casos de teste a serem reexecutados tanto para programas de grande porte, como o SPACE, quanto para programas pequenos, como os programas UNDX. Para todos os programas a técnica foi capaz de selecionar conjuntos de casos de teste de regressão com $100 \%$ de eficácia. Os valores de precisão e recall para os programas não foram muito diferentes.

Para programas mais complexos, a análise das modificações pode aumentar muito os custos de aplicação dessa técnica, uma vez que é necessário ter conhecimento das modificações realizadas para selecionar os casos de teste modification_traversing.

Tabela 3.28-Média para os conjuntos minimizados e priorizados dos programas UNLX*.

\begin{tabular}{|c|c|c|c|c|}
\hline 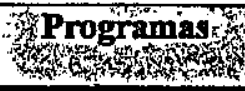 & IX Redigho de & 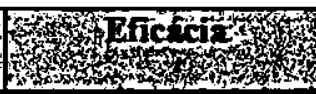 & W & H \\
\hline Cal & $83,926 / 83,954$ & $100 / 100$ & $59,815 / 90,625$ & $16,293 / 18,159$ \\
\hline Checkeg & $92,617 / 92,107$ & $100 / 100$ & $43,613 / 91,270$ & $10,592 / 9,828$ \\
\hline Comm & $95,608 / 95,655$ & $100 / 100$ & $48,636 / 72,778$ & $9,737 / 13,740$ \\
\hline Look & $88,558 / 98,346$ & $100 / 100$ & $73,315 / 93,750$ & $27,243, / 21,435$ \\
\hline Uniq & $98,395 / 97,787$ & $100 / 100$ & $78,191 / 88,889$ & $1,838 / 3,723$ \\
\hline
\end{tabular}

* conjuntos minimizados/conjuntos priorizados

Para o experimento conduzido por Wong et al. (1997b), descrito no capítulo anterior, utilizando a técnica baseada em Mutação Seletiva nos programas UNIX, todos os conjuntos de teste de regressão reduzidos foram capazes de revelar os defeitos com uma grande redução do número de casos de teste a serem reexecutados. Ex.: Para o programa $\mathrm{CAL}$, em média cada conjunto de teste de regressão reduzido continha 17,4 casos de teste e a eficácia foi de $100 \%$ para todos os conjuntos com relação aos defeitos disponiveis (Tabela 3.29). Os valores de precisão e recall não foram apresentados por Wong et al., assim como os resultados para cada um dos demais programas UNLX.

Aplicando a técnica baseada em Mutação Seletiva no programa SPACE, muitos casos de teste foram selecionados para serem reexecutados. Isso ocorreu devido a complexidade do programa SPACE. O conjunto de casos de teste selecionado para o programa SPACE continha 229 casos de teste, muito mais casos de teste que a média de 17,4 casos de teste nos conjuntos do programa $\mathrm{Cal}$. Os valores médio de precisão e recall para os conjuntos selecionados foram $8,626 \%$ e $36,281 \%$, respectivamente. 
Tabela 3.29 - Número de casos de teste selecionados para os conjuntos reduzidos com relaçăo ao critério MUT-A para o programa Cal.

\begin{tabular}{|c|c|}
\hline 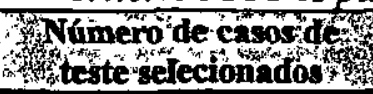 & Wumerode dercitos \\
\hline 20 & 20 \\
\hline 16 & 20 \\
\hline 17 & 20 \\
\hline 16 & 20 \\
\hline 18 & 20 \\
\hline
\end{tabular}

\subsubsection{Avaliação das Técnicas de Teste de Regressão segundo o Framework de Rothermel e Harrold}

Seguindo o framework, cada uma das técnicas foram avaliadas. Relembrando, as seguintes características são avaliadas pelo framework:

Inclusão - mede a extensão com a qual a técnica escolhe casos de teste que fazem com que $\mathbf{P}$ ' produza um saida diferente de $\mathbf{P}$ e revela os defeitos, casos de teste modification_revealing.

$>$ Precisão - mede a habilidade do técnica em evitar casos de teste que não fazem com que $\mathbf{P}$ ' produza um saida diferente de $\mathbf{P}$, casos de teste non_modification_revealing.

$>$ Eficiência - mede o custo computacional gasto pela técnica.

$>$ Generalidade - mede a habilidade da técnica em manipular linguagens diversas.

A precisão avaliada aqui e aquela avaliada na seção 3.2 têm o mesmo conceito, no entanto, calculam valores diferentes. Enquanto que a precisão do framework calcula a habilidade da técnica em evitar casos de teste que não causam comportamentos diferentes entre os programas original e modificado, ou seja, casos de teste non_modification_revealing, a outra calcula a porcentagem de casos de teste que são capazes de revelar um comportamento diferente entre os programas original e modificado. Assim, para diferenciar, a precisão definida pelo framework é referenciada como precisao_F.

Primeiramente, a avaliação dos resultados da Técnica baseada em Modificação é apresenta, e, em seguida, a avaliação dos resultados da Técnica baseada em Mutação Seletiva. 
A Técnica baseada em Modificação foi capaz de selecionar um menor número de casos de teste para serem reexecutados considerando os cinco programas utilizados. $O$ mecanismo de priorização utilizado possibilitou tornar a técnica mais segura tanto com relação à Técnica baseada em Mutação Seletiva quanto com relação ao mecanismo de minimização.

\section{Inclusão e Precisão_F}

Para o programa Cal, a precisão_F para os conjuntos minimizados é de $66,667 \%$ para os conjuntos $\mathrm{T}^{\prime \prime} \mathrm{MD1}, \mathrm{T}$ " ${ }_{\mathrm{MDS}}$ e $\mathrm{T}^{\text {" }}{ }_{\mathrm{MD}}$ e de $100 \%$ para os demais conjuntos. Assim, $85 \%$ dos conjuntos não possuem casos de teste que não revelam defeitos, ou seja, casos de teste non_modification_revealing. A precisão_F para os conjuntọs priorizados é de $100 \%$ para todos os conjuntos. Assim, todos os conjuntos não possuem casos de teste que não revelam defeitos. $\mathrm{Na}$ média, para os conjuntos minimizados a inclusão é de $16,293 \%$ e para os conjuntos priorizados é de $18,159 \%$.

Como para o programa Checkeq todos os casos de teste disponíveis no conjunto original são capazes de revelar pelo menos um defeito do conjunto utilizado, a precisão_F não foi calculada para os conjuntos minimizados e priorizados. A inclusão dos conjuntos minimizados, em média, foi de $10,592 \%$, e $9,828 \%$, em média, para os conjuntos priorizados. A inclusão para os minimizados e priorizados, com relação a cada defeito do programa, pode ser observada nas Tabelas 3.13 e 3.14 .

Para o programa Comm, a média de inclusão para os conjuntos minimizados foi de $9,737 \%$, e $13,740 \%$ para os conjuntos priorizados. A inclusão para cada um dos defeitos, com relação aos conjuntos minimizados e priorizados, pode ser observada nas Tabelas 3.15 e 3.16, respectivamente. Já a precisão_F foi de $100 \%$ para os conjuntos priorizados e $98,148 \%$ para os conjunto minimizados. Nesse casos, somente para o defeito $\mathrm{Dl}$ a técnica não foi de omitir todos os casos non_modification_revealing, sendo a precisão_F para esse conjunto de $66,667 \%$. O programa Comm é o programa que possui um menor número de casos de teste que não revelam um comportamento diferente entre o programa original e o programa modificado. Como esse programa possui o maior conjunto de casos de teste, as chances dos defeitos serem revelados são maiores. 
Para o programa Look, a inclusão obteve uma média de $27,243 \%$ para os conjuntos minimizados e $21,435 \%$ para os conjuntos priorizados. Os valores de inclusão para cada defeito podem ser observados nas Tabelas 3.17 e 3.18 para os conjuntos minimizados e priorizados, na coluna dos valores do recall. Para todos os conjuntos priorizados, a precisão_F foi de $100 \%$, enquanto que para os conjuntos minimizados, essa média foi de $99,583 \%$, sendo de $93,333 \%$ para o conjunto minimizado relacionado ao defeito $\mathrm{D} 1$.

O Uniq foi o programa que obteve uma menor média de inclusão com relação aos outros quatro programas. Para os conjuntos minimizados, essa média foi de $1,838 \%$, e para os conjuntos priorizados a média foi de $3,723 \%$. A inclusão para cada um dos defeitos pode ser observada nas Tabelas 3.19 e 3.20, para os conjuntos minimizados e priorizados respectivamente. No caso da precisão_F, para todos conjuntos priorizados essa medida foi de $100 \%$. Já para os conjuntos minimizados, a média foi de $99,647 \%$. O programa Uniq foi aquele com um maior número de conjuntos minimizados que possuíam casos de teste non_modification_revealing. Para os defeitos D1, D12 e D15, a precisão_F foi de 99,091\%, e para os defeitos D7 e D8, a precisão_F foi de $98,182 \%$. Como observado anteriormente, vide Tabela 3.3, o programa Uniq é aquele que possui um maior número de casos de teste que não revelam um comportamento diferente entre o programa original e o programa modificado, 110 casos de teste no total.

Eficiência: o custo de aplicação da técnica foi comprometido pelo fato de que foi necessário estabelecer o trace de execução de cada um dos casos de teste disponíveis para os cinco programas a fim de verificar quais casos de teste executavam quais modificações. No entanto, esse custo não foi tão elevado, pois se utilizou a ferramenta PROTEUM/IM que estabelece o trace de execução de um caso de teste, como já mencionado. O esforço maior foi despendido na tarefa de identificar em quais blocos de comandos as modificações foram realizadas. Comparando o trace de execução de cada caso de teste com o bloco no qual a modificação estava, foi possível definir os conjuntos de casos de teste modification_traversing para cada uma das modificações. Essa tarefa foi realizada através de scripts em $C$-shell do Unix. Como para cada uma das modificações um subconjunto de casos de teste de regressão foi definido, o custo também foi comprometido.

Generalidade: a técnica não depende da linguagem do programa em teste, nem mesmo da técnica aplicada para o teste. Independentemente das modificações realizadas, a técnica é capaz de selecionar um conjunto de casos de teste que as executem, sejam elas de remoção, alteração ou inserção de comandos, intra ou interprocedurais. Assim, qualquer ferramenta de teste que seja capaz de fornecer o trace de execução de um caso de teste pode apoiá-la 
computacionalmente, seja uma ferramenta que apóia o teste estrutural ou o teste de mutação. Por outro lado, o testador deve ter um prévio conhecimento de onde as modificações foram realizadas, pois não é possível saber se um caso de teste passa por uma modificação sem saber onde essa modificação está. Para programas complexos, essa tarefa despende bastante esforço, pois programas complexos possuem grafos de fluxo de controle complexos. No entanto, esse custo pode ser amenizado com a utilização de ferramentas automatizadas que realizam essa tarefa.

\section{$\rightarrow$ Técnica baseada em Mutação Seletiva}

A Técnica baseada em Mutação Seletiva obteve uma menor redução do conjunto de casos de teste a serem reexecutados durante o teste de regressão. Esse fato ocorreu devido à complexidade do programa SPACE e de suas modificações. A técnica também fó menos eficaz em evitar casos de teste que não revelam defeitos.

\section{Inclusão e Precisão_F}

A precisão_F para o conjunto T’ AM_adequado selecionado é de 81,225\%, ou seja, 411, dos 506 casos de teste non_modification_revealing, foram omitidos do conjunto de teste de regressão T'. A inclusão para o conjunto T' com relação ao conjunto original foi de $27,126 \%$. Considerando cada um dos defeitos disponíveis, em média, a inclusão foi de $36,281 \%$. Os valores da inclusão para cada defeito estão na Tabela 3.27, coluna dos valores de recall.

Eficiência: como o programa utilizado é de grande porte, a aplicação da técnica demandou muito custo computacional, na qual muitos mutantes foram gerados e executados pela ferramenta PROTEUM mesmo utilizando-se um subconjunto de operadores de mutação.

Generalidade: essa técnica também não depende da linguagem do programa em teste, no entanto, depende do critério de teste utilizado, no caso a Mutação Seletiva. Assim, para a aplicação computacional dessa técnica é necessário ter disponível uma ferramenta de teste que apóie a mutação seletiva. Além disso, é necessário também ter um prévio conhecimento do critério de teste e dos conceitos de teste de mutação. $O$ custo de aplicação da técnica foi amenizado pelo fato de que somente um conjunto AM_adequado foi definido para o teste de regressão de todas as modificações disponíveis. Além disso, o testador não necessariamente precisa saber onde as modificações foram realizadas para definir o subconjunto de teste de regressão a partir da mutação seletiva. 
As Tabelas 3.30 e .3 .31 sintetizam os resultados obtidos com a aplicação das técnicas de teste de regressão.

Tabela 3.30 - Média dos resultados de redução de tamanho, eficácia, inclusão e precisão_F para os conjuntos minimizados e priorizados do programas UNIX*.

\begin{tabular}{|c|c|c|c|c|}
\hline Wh Prompanas & 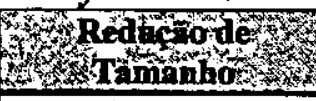 & Y) & (X) & W \\
\hline Cal & $83,926 / 83,954$ & $100 / 100$ & $16,293 / 18,159$ & $66,667 / 85$ \\
\hline Checkeq & $92,617 / 92,107$ & $100 / 100$ & $10,592 / 9,828$ & -- \\
\hline Comm & $95,608 / 95,655$ & $100 / 100$ & $9,737 / 13,740$ & $98,148 / 100$ \\
\hline Look & $88,558 / 98,346$ & $100 / 100$ & $27,243 / 21,435$ & $99,583 / 100$ \\
\hline Uniq & $98,395 / 97,787$ & $100 / 100$ & $1,838 / 3,723$ & $99,647 / 100$ \\
\hline
\end{tabular}

*conjuntos minimizados/conjuntos priorizados

Tabela 3.31 - Média dos resultados de redução de tamanho, eficácia, inclusão e precisão_F para o programa SPACE.

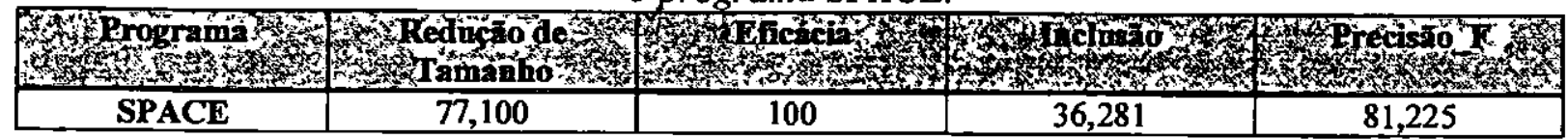

\subsection{Considerações Finais}

Neste capítulo foram apresentados os experimentos realizados para avaliar a aplícação de duas técnicas de teste de regressão: Técnica baseada em Modificação e Técnica baseada em Mutação Seletiva.

Com base nos experimentos realizados, um fato que deve ser ressaltado é que a complexidade do programa em teste compromete a eficiência da técníca. Além disso, a dependência do critério de teste também compromete a aplicação da técnica. Observa-se também pelos resultados obtidos que, dependendo dos mecanismos de minimização ou priorização aplicados, é possível deixar uma técnica mais segura, garantindo que casos de teste que não sejam modification_revealing não sejam selecionados. Outro fato observado, é que os mecanismos de minimização e priorização podem ser aplicados em qualquer técnica de teste de regressão, pois ambos não dependem da técnica. Para comprovar essa possibilidade, Rothermel et al. (1999) descrevem diversos experimentos utilizando-se diferentes mecanismos de priorização para auxiliar na seleção de subconjuntos de casos de teste de regressão. 


\section{Conclusões e Trabalhos Futuros}

Ressaltou-se neste trabalho a importância das atividades de teste na fase de manutenção de um software. Outro ponto importante também pertinente para o teste de regressão é a redução de custo de sua aplicação. Como se demonstrou por meio dos experimentos realizados, o teste de regressão é tão caro quanto o teste de software realizado durante o processo de desenvolvimento. Assim, diversas técnicas são propostas na literatura para reduzir os custos do teste de regressão. No entanto, a diversidade de técnicas de teste de regressão dificulta a escolha de uma delas. Diversos estudos empíricos são realizados para avaliar e comparar essas técnicas com o intuito de estabelecer estratégias de teste de regressão menos onerosas para os testadores e facilitar a escolha de uma delas.

Com base nos experimentos realizados por Wong et al. (1997a, 1997b) para avaliar as técnicas de teste de regressão baseada em Modificação e Mutação Seletiva com o programas SPACE e UNIX, respectivamente, este trabalho avaliou e comparou empiricamente essas duas técnicas de teste de regressão, utilizando a técnica baseada em Modificação para testar os programas UNIX e a técnica baseada em Mutação Seletiva para testar o programa SPACE.

Observou-se com a realização desses experimentos que ambas as técnicas se mostraram eficientes em revelar os defeitos nos programas modificados com um número bastante reduzido de casos de teste a serem reexecutados. No entanto, esse conjunto não foi tão reduzido considerando o experimento conduzido com o programa SPACE e a técnica baseada em Mutação Seletiva, pois o programa possui uma alta complexidade. A complexidade de um programa comprometer a eficácia da técnica em selecionar um menor número de casos de testes a serem reexecutados.

Com a aplicação das técnicas descritas neste trabalho, foi possível concluir que técnicas baseadas em selecionar casos de teste que cobrem modificações são mais eficazes em reduzir o 
conjunto de casos de teste a serem reexecutados e em revelar possíveis defeitos do que as técnicas baseadas no comportamento. Estudos realizados por Rothermel e Harrold (1997) e Wong et al. (1997b) também comprovam que técnicas que baseiam-se na cobertura são mais eficazes em revelar defeitos. Assim, um caso de teste que executa uma modificação tem maiores chances de revelar um possível defeito. No entanto, nem todo caso de teste que executa uma modificação é capaz de revelar defeitos.

Outro ponto importante é um mecanismo de priorização são eficazes em revelar defeitos reduzindo bastante o número de casos de teste a serem reexecutados. Estudos empíricos realizados por Rothermel et al. (1999) com diferentes mecanismos de priorização demonstram a eficácia desses mecanismos em revelar defeitos com um número reduzido de casos de teste. Segundo esses estudos, o mecanismo de priorização mais eficaz é aquele que seleciona os casos de teste que mais revelavam defeitos, sendo esse o mesmo mecanismo utilizado neste trabalho para aplicar a Técnica baseada em Modificação. No entanto, nesse caso é necessário ter um prévio conhecimento das modificaçōes realizadas.

\subsection{Trabalhos Futuros}

Para ilustrar possiveis trabalhos futuros podem-se citar:

$>$ Ampliar o universo de programas utilizados para a comparação das técnicas.

$>$ Avaliar o custo de aplicação das técnicas de acordo com o modelo de custo proposto por Leung e White (1991)

$>$ Utilização de outros mecanismos de priorização para a Técnica baseada em Modificação. Rothermel et al. (1999) avaliam empiricamente diversos mecanismos de priorização, como já mencionado. Alguns desses mecanismos poderiam ser utilizados com o intuito de, além de melhorar a aplicação da Técnica baseada em Modificação, complementar os estudos realizados por Rothermel.

$>$ Utilização do conjunto de operadores essenciais de mutação para o Critério Mutação de Interface, definido por Vincenzi em seu trabalho (1998), para a Técnica baseada em Mutação Seletiva. Assim, a invés de avaliar o quanto que um conjunto de casos de teste AM_adequado é adequado ao programa original e também adequado às suas versões modificadas, avaliar-se-ia o quanto que um conjunto de casos de teste adequado ao 
critério Mutação de Interface com relação ao programa original é também adequado às suas modificações.

$>$ Explorar essas técnicas no contexto de reengenharia reversa. 

Abdullah, K., Kimble, J., White, L.; Correcting for Unreliable Regression Integration Testing, IEEE Proceedings of the $17^{\text {th }}$ International Conference on Software Maintenance, Outubro, 1995.

Acree, A., Budd, T. A., DeMillo, R. A., Lipton, R. J., Sayward, F. G.; Mutation Analysis, Relatório Técnico GIT-ICS-79/08, Georgia Institute of Technology, Setembro, 1979.

Barbosa, E. F.; Uma Contribuição para Determinação de um Conjunto Essencial de Operadores de Mutação para o Teste de Programas C, Dissertação de Mestrado, ICMC-USP, Novembro, 1998.

Bates, S., Horwitz, S.; Incremental Program Testing Using Program Dependence Graphs, Proceedings of the $20^{\text {th }}$ ACM Symposium on Principles of Programming Languages, Janeiro, 1993.

Biezer, B.; Software Testing Techniques, $2^{\mathbf{a}}$ Edição, Van Nostrand Reinhold, New York, 1990.

Binkley, D.; Reducing the Cost of Regression Testing by Semantics Guided Test Case Selection, IEEE Proceedings of the $17^{\text {th }}$ International Conference on Software Maintenance, Outubro, 1995.

Budd, T. A., DeMillo, R. A., et al; Theoretical and Empirical Studies on Using Programs Mutation to Test the Functional Correctness of Programs, Proceedings of the $7^{\text {th }}$ ACM Symposium on Principles of Programming Languages, Janeiro, 1980a.

Budd, T. A.; Mutation Analysis of Programs Test Data, Tese de Doutorado, Yale University, Maio, 1980b.

Budd, T. A.; Mutation Analysis: Ideas, Examples, Problems and Prospects, Computer Program Testing, North-Holand Publishing Company, 1981.

Chaim, M. L.; Poke-tool - Uma Ferramenta para Suporte ao Teste Estrutural de Programas Baseado em Análise de Fluxo de Dados, Dissertação de Mestrado, DCA/FEEC/UNICAMP, 1991.

Chen, Y., Rosenblum, D.S., Vo, K.; TestTube: A System for Selective Regression Testing, IEEE Proceedings of the $16^{\text {th }}$ International Conference on Software Engineering, Maio, 1994.

Coward, P.; A Review of Software Testing, Information and Software Technology, Abril, 1988.

Delamaro, M. E.; Proteum - Um Ambiente de Teste Baseado na Análise de Mutantes, Dissertação de Mestrado, ICMSC/USP, Outubro, 1993

Delamaro, M. E.; Mutaçao de Interface: Um Critério de Adequação Interprocedural para o Teste de Integraçao, Tese de Doutorado, IFSC/USP, 1997a.

Delamaro, M. E. ,Maldonado, J. C.; Interface Mutation : An Approach for Integration Testing, Workshop do Projeto Validação de Teste de Sistemas de Operação, Janeiro, $1997 \mathrm{~b}$.

Delamaro, M. E., Maldonado, J. C.; Interface Mutation : A Case Study, Workshop do Projeto Validação de Teste de Sistemas de Operação, Janeiro, 1997c.

DeMillo, R. A., Lipton, R. J., Sayward, F. G.; Hints on Test Data Selection: Help for the Practicing Programmer, IEEE Computer, Abril, 1978. 
DeMillo, R. A.; Mutation Analysis as a Tool for Software Quality Assurance, Proceedings of the COMPSAC80, Outubro, 1980.

DeMillo, R. A.; Software Testing and Evaluation, The Benjamin/Cummings Publishing Company, 1987.

DeMillo, R. A., Mathur, A. P.; On the use of Software Artifacts to Evaluate the Effectiveness of Mutation Analysis for Detecting Errors in Production Software, Proceedings of the $13^{\text {th }}$ Minnowbrook Workshop on Software Engineering, Julho, 1990.

Forgács, I., Takács, E.; Mutation-Based Regression Testing, Networked Computer Science Technical Reference Library, Maio, 1997.

Frankl P. G.; The Use of Data Flow Information for the Selection and Evaluation of Software Test Data, Tese de Doutorado, New York University, Outubro, 1987.

Granja, I.; Uma Ferramenta de Apoio ao Teste de Regressao, Dissertação de Mestrado, DCAFEEC-UNICAMP, Dezembro, 1997.

Granja, I., Jino, M.; Techniques for Regression Testing: Selecting Test Case Sets Taylored to Possibly Modified Functionalities, Proceedings of the $3^{\text {rd }}$ European Conference on Software Maintenance and Reengineering, Março, 1999.

Graves, T., Harrold, M., Kim, J., Porter, A., Rothermel, G.; An Empirical Study of Regression Test Selection Techniques, Proceedings of the $20^{\text {th }}$ International Conference on Software Engineering, IEEE, Abril, 1998.

Garey, M. R., Johnson, D. S.; Computers and Intractability: $A$ Guide to the Theory of NPCompleteness, V.Klee, Ed. Freeman, 1979.

Hartmann, J., Robson, D.; Techniques for Selective Revalidation, IEEE Software, Janeiro, 1990.

Harrold, M. J., Gupta, R. and Soffa, M. L.; A Methodology for Controlling the Size of a Test Suite, ACM Transactions on Software Engineering and Methodology, Julho, 1993.

Harrold, M. J., Rothermel, G.; Aristotle: A System for Research on and Development of ProgramAnalysis-Based Tools, Relatório Técnico OSU-CISRC-3/97-TRI7, Ohio State University, Março, 1997.

Howden, W. E.; The Theory and Practice of Functional Testing, IEEE Software, Setembro, 1985.

Koening, A.; C Traps and Pitfalls, Addison-Wesley, 1988.

Lehman, M. M.; Process Improvement - The Way Forward, Anais do X Simpósio Brasileiro de Engenharia de Software, Outubro, 1996.

Leung, H. K. N., White, L.; A Cost Model to Compare Regression Test Strategies, IEEE Proceedings of the $13^{\text {th }}$ International Conference on Software Maintenance, Outubro, 1991.

Maldonado, J. C.; Critérios Potenciais Usos: Uma Contribuição para o Teste Estrutural de Software, Tese de Doutorado, DCA/FEEC/UNICAMP, Julho, 1991.

Mathur, A. P.; On the Relative Strengths of Data Flow and Mutation Testing, Proceedings of the $9^{\text {th }}$ Annual Pacific Northwest Software Quality Conference, 1991.

Mathur, A. P., Wong, W. E.; Evaluation of The Cost Alternate Mutation Strategies, VII Simpósio Brasileiro de Engenharia de Software, Outubro, 1993.

Mathur, A. P., Wong, W. E.; An Empirical Comparison of Data Flow and Mutation-Based Test Adequacy Criteria, The Journal of Software Testing, Verification and Reliability, Março, 1994.

Mayrhauser, A. v., Mraz, R. T., Walls, J.; Domain Based Regression Testing, IEEE Proceedings of the $16^{\text {th }}$ International Conference on Software Maintenance, Setembro, 1994. 
Myers, G. J.; The Art of Software Testing, John Wiley \& Sons, 1979.

Offut, A. J., Rothermel, G., Zapt, C.; An Experimental Evaluation of Selective Mutation, Proceedings of the $15^{\text {th }}$ International Conference on Software Engineering, Maio, 1993.

Offut, A. J., Pan, J., Tewary, K., Zhang, T.; An Experimental Evaluation of Data Flow and Mutation Testing, Software Practice and Experience, Fevereiro, 1996a.

Offut, A. J., Lee, A., Rothermel, G., Untch, R. H., Zapf, C.; An Experimental Determination of Sufficient Mutant Operators, ACM Transaction on Software Engineering Methodology, Abril, 1996b.

Ostrand T. J., Weyuker E. J.; Using Data Flow Analysis for Regression Testing, Proceedings of the $6^{\text {th }}$ Annual Pacific Northwest Software Quality Conference, Setembro, 1988.

Pressman, R. S.; Software Engineering - A Practitioner's Approach, $3^{*}$ Edição, McGraw Hill, 1992.

Rapps, S., Weyuker, E. J.; Data Flow Analysis Techniques for Test Data Selection, Proceedings of the International Conference on Software Engineering, Setembro, 1982.

Rapps, S., Weyuker, E. J.; Selecting Software Test Data Using Data Flow Information, IEEE Transactions on Software Engineering, Abril, 1985.

Rosenblum, D. S., Weyuker, E. J.; Using Coverage Information do Predict the Cost-Effectiveness of Regression Testing Strategies, IEEE Transactions on Software Engineering, Marco, 1997.

Rothermel, G., Harrold, M. J.; Analyzing Regression Test Selection Techniques, IEEE Transactions on Software Engineering, Agosto, 1996.

Rothermel, G., Harrold, M. J.; A Safe, Efficient Regression Test Selection Technique, ACM Transactions on Software Engineering and Methodology, Abril, 1997.

Rothermel, G., Harrold M. J.; Empirical Studies of a Safe Regression Test Selection Technique, IEEE Transactions on Software Engineering, Junho, 1998.

Rothermel, G., Untch, R. H., Chu, C., Harrold, M. J., Test Case Prioritization: An Empirical Study, IEEE Proceedings of the $21^{\text {st }}$ International Conference on Software Maintenance, Setembro, 1999.

Souza, S. R. S.; Avaliação do Custo e Eficácia do Critério Análise de Mutantes na Atividade de Teste de Programas, Dissertação de Mestrado, ICMSC-USP, Junho, 1996.

Souza, S. R. S.; Análise de Técnicas Seletivas para o Teste de Regressão, Exame de Qualificação para obtenção do Título de Doutor, IFSC-USP, Outubro, 1997.

Tullio, R. R.; Estudos Empiricos de Critério de Teste de Software com Ênfase no Critério Análise de Mutantes, Projeto de Iniciação Científica em andamento, 1999.

Varadan G. S.; Trends in Reliability and Test Strategies, IEEE Software, Maio, 1995.

Vergílio, S. R.,Critérios Restritos de Teste de Software: Uma Contribuição para Gerar Dados de Teste mais Eficazes, Tese de Doutorado, DCA/FEEC/UNICAMP, Julho, 1997.

Vilela, P. R. S.; Uma ferramenta para Auxilio Visual ao Teste e Depuração de Programas, Dissertação de Mestrado, DCA/FEE/UNICAMP, Março, 1994.

Vilela, P. R. S., Maldonado, J. C., Jino, M.; Program Graph Visualization: An Approach without Replacement, Software Practice \& Experience, Abril, 1996.

Vincenzi, A. M. R.; Subsídios para o Estabelecimento de Estratégias de Teste Baseadas na Técnica de Mutação, Dissertação de Mestrado, ICMC-USP, Novembro, 1998. 
White, L. J., Leung, H. K. N. ; A Firewall Concept for Both Control-Flow and Data-Flow in Regression Integration Testing, IEEE Proceedings of the $14^{\text {th }}$ International Conference on Software Maintenance, Novembro, 1992.

Wong, W. E.; On Mutation and Data Flow, Tese de Doutorado, Software Engineering Research Center - Purdue University, Dezembro, 1993.

Wong, W. E., Maldonado, J. C., Delamaro, M. E., Mathur, A. P.; Constrained Mutation in C Programs, Anais VIII Simpósio Brasileiro de Engenharia de Software, Outubro, 1994a.

Wong, W. E., Mathur, A. P., Maldonado, J. C.; Mutation versus All-uses: An Empirical Evaluation of Cost, Strength and Effectiveness, $1^{\text {st }}$ IFIP/SQI - International Conference on Software Quality and Productity (ICSQP'94): Theory, Practice, Education and Training, Dezembro, 1994b.

Wong, W. E., Mathur, A. P, London, S.; Effect of Test Set Minimization on the Fault Detection Effectiveness of the All-Uses Criterion, Relatório Técnico SERC-TR-152-P, Software Engineering Research Center, Purdue University, Abril, $1994 c$.

Wong, W. E., Mathur, A. P.; Fault detection effectiveness of mutation and data flow testing, Software Quality Journal, Março, 1995a.

Wong, W. E., Mathur, A. P.; Reducing the cost of mutation testing: An empirical study, The Journal of Systems and Software, Dezembro, 1995b.

Wong, W. E., Horgan, J. R., London, S., Agrawal, H.; A Study of Effective Regression Testing in Practice, Proceedings of the $8^{\text {th }}$ IEEE International Symposium on Software Reliability Engineering, Novembro, 1997a.

Wong, W. E., Maldonado, J. C., Delamaro, M. E.; Reduncing the Cost of Regression Testing by Using Selective Mutation, Anais VIII Conferência Internacional de Tecnologia de Software: Qualidade de Software, Junho, $1997 \mathrm{~b}$.

Wong, W. E., Maldonado, J. C., Delamaro, M. E., Souza, S. R. S.; Use of Proteum to Accelerate Mutation Testing in C Programs. Proceedings of the $3^{\text {rd }}$ ISSAT International Conference on Reliability and Quality in Design, Anaheim, Março, 1997c.

Yau, S. S., Kishimoto, Z.; A Method for Revalidating Modified Programs in the Maintenance Phase, Proceedings IEEE Compsac, 1987. 


\section{Operadores de Mutação}

A seguir os operadores de mutação utilizados por Wong et al. (1997b) em seus experimentos (Quadro A.1) e os operadores essenciais de mutação utilizados neste trabalho (Quadro A.2) são descritos.

Quadro A.1 - Operadores de Mutação utilizados por Wong et al.

\begin{tabular}{|c|c|}
\hline 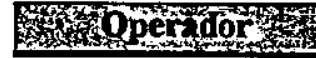 & $f_{1}$ \\
\hline VDTR & Atribui os valores negativo, positivo e zero para cada referência escalar \\
\hline VTWD & Substitui referência escalar por seu valor antecessor ê sucessor \\
\hline SSDL & Retira um comando de cada vez do programa \\
\hline SMVB & Move ' $\}$ ' para cima e para baixo quando possivel \\
\hline SBRn & $\begin{array}{l}\text { Troca o comando continue ou break por uma função } \\
\text { break_out_of_level_n(J) sendo que } J \text { varia de acordo com o múmero de laços } \\
\text { aninhados. Essa funcão forca a interrupcão dos J lacos externos }\end{array}$ \\
\hline SCRn & $\begin{array}{l}\text { Troca o comando continue ou break por uma função } \\
\text { continue_out_of_level_n(J) sendo que } \mathrm{J} \text { varia de acordo com o número de } \\
\text { laços aninhados. Essa função força a transferência do programa para o final de } \\
\mathrm{J} \text { laços acima }\end{array}$ \\
\hline Vprr & $\begin{array}{l}\text { Substitui as referências a apontadores por variáveis escalares, globais e locais } \\
\text { do programa }\end{array}$ \\
\hline Vtrr & $\begin{array}{l}\text { Substitui as referências a estruturas e uniōes por variáveis escalares, globais e } \\
\text { locais do programa }\end{array}$ \\
\hline CRCR & Troca constantes por: $0,1,-1$, dependendo do tipo de referência \\
\hline OLLN & Troca operador lógico por outro operador lógico \\
\hline OLNG & Insere negação lógica em condiç̃es compostas \\
\hline ORRN & Troca operador relacional por outro operador relacional \\
\hline
\end{tabular}

Quadro A.2 -Operadores Essenciais de Mutação.

\begin{tabular}{|c|c|}
\hline Operador & W \\
\hline SWDD & Troca o comando while por do while \\
\hline SMTC & Interrompe a execução do laco após 2 execucōes \\
\hline SSDL & Descrito acima \\
\hline OLBN & Troca operador lógico por operador bitwise \\
\hline ORRN & Descrito acima \\
\hline VTWD & Descrito acima \\
\hline VDTR & Descrito acima \\
\hline $\mathrm{Cccr}$ & Troca constantes por outra constante \\
\hline Cosr & Troca referências escalares por constantes \\
\hline
\end{tabular}



Apêndice B

\section{Versões Modificadas dos Programas do UNIX e Ativação do erros do Programa SPACE}

Neste Apêndice encontram-se as versões modificadas geradas para os programas UNIX utilizadas nos experimentos e o processo de ativação de erros do programa SPACE. Utilizou-se a primitiva diff do UNIX para diferenciar a versão original de cada programa de suas versōes. A primitiva diff tem como entrada, além de parâmetros, ọ arquivos (diretórios) que devem ser comparados. Após compará-los, a primitiva retorna a linha na qual os arquivos (diretórios) são diferentes e o conteúdo dessas linhas. A primitiva possui a seguinte sintaxe:

diff [conjunto de parâmetros] arq1 arq $2 /$ [conjunto de parâmetros] dir 1 dir2

Versões Modificados dos Programas UNIX

\section{Programa CAL}

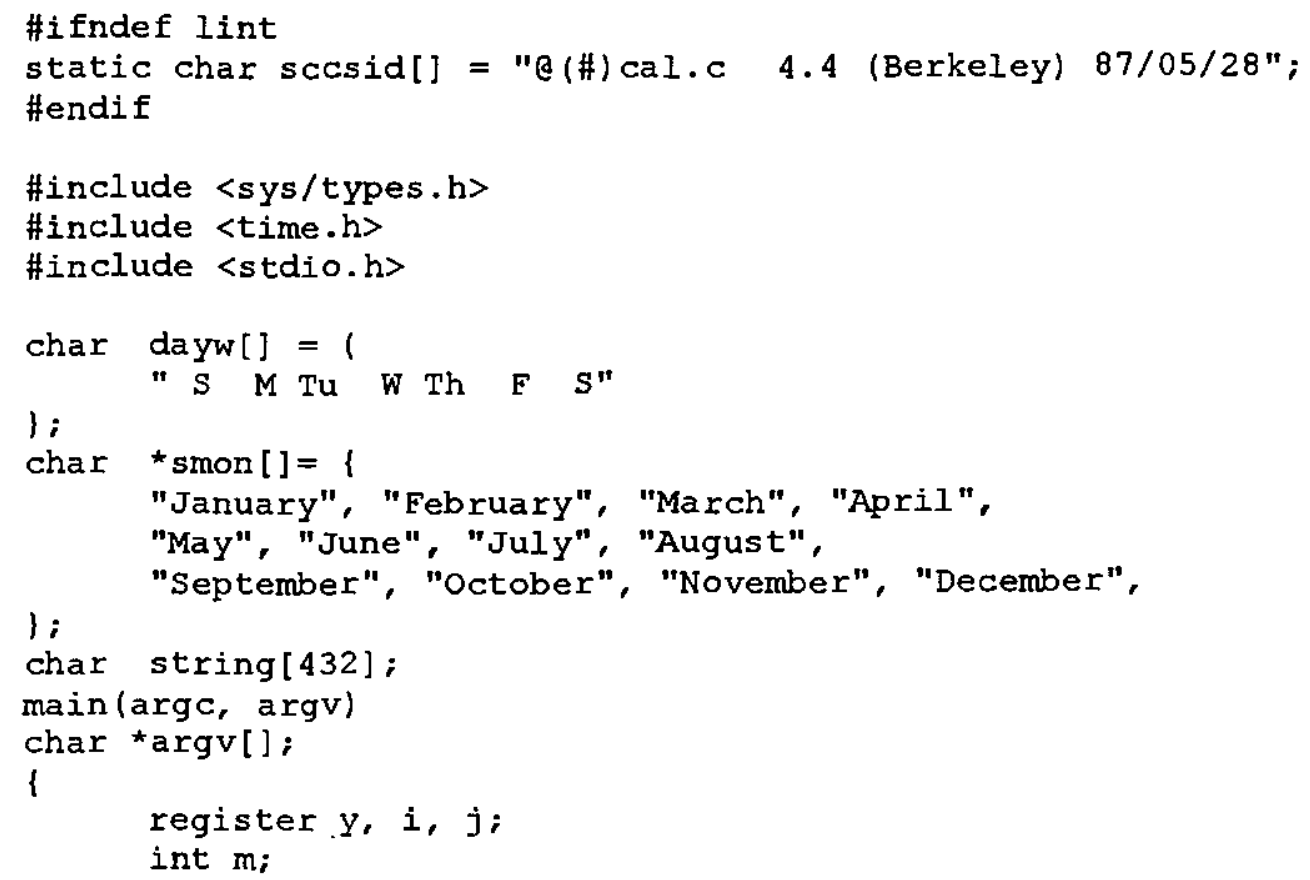




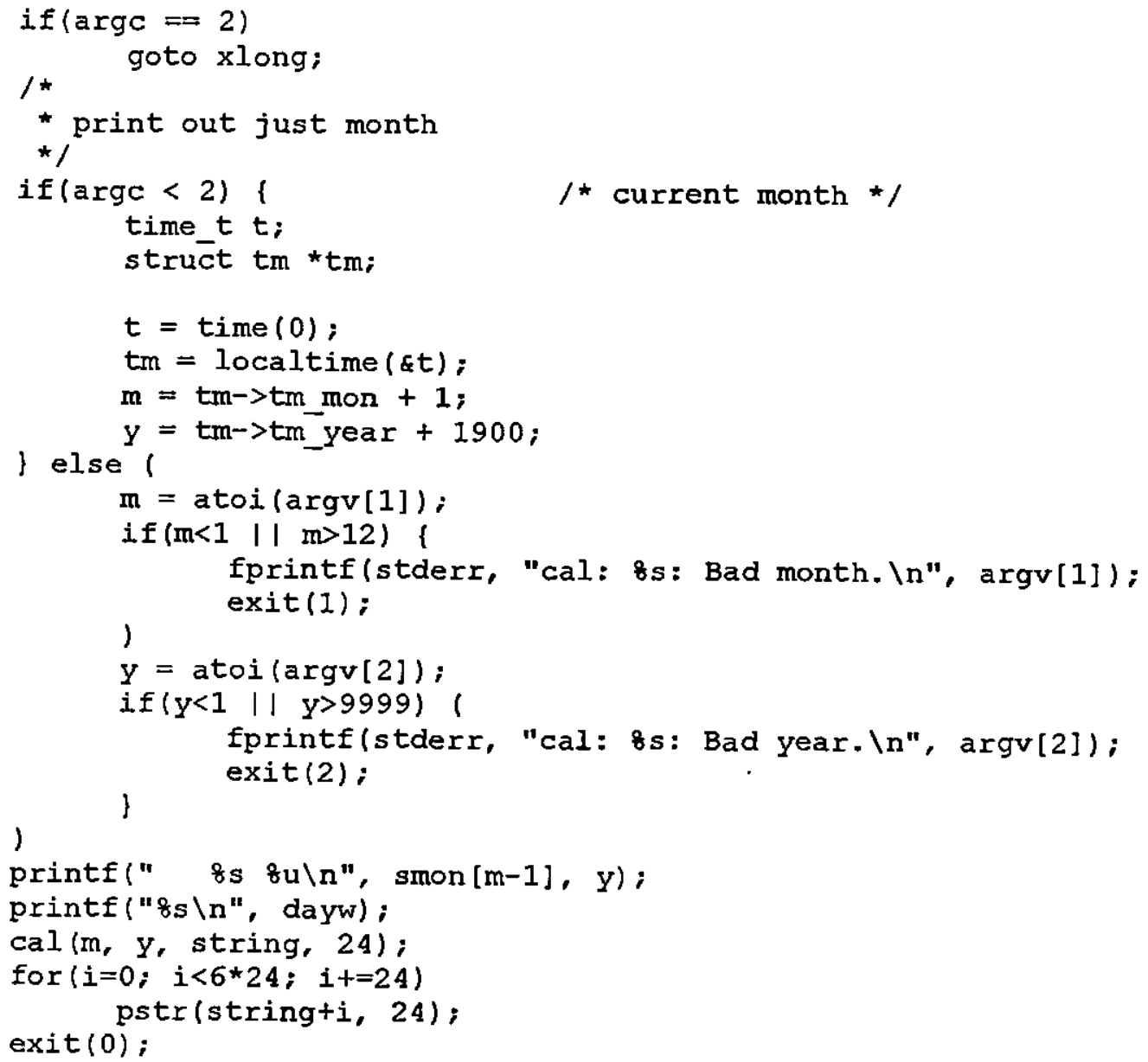

xlong :

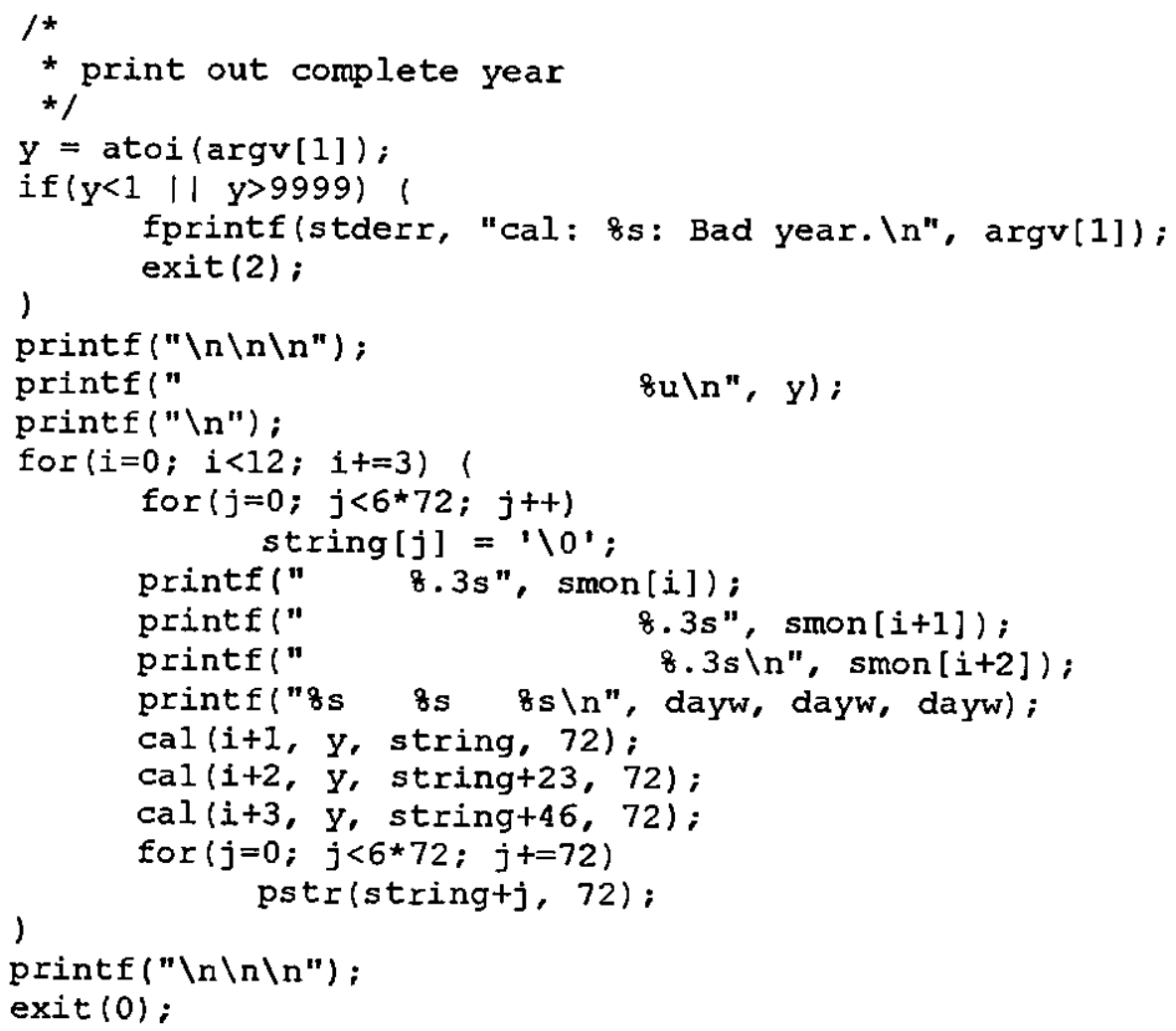


)

pstr(str, n)

char *str;

I

register $i$;

register char *s;

$s=s t r$

$i=n$;

while (i--)

if (*st+ $=$ " $\backslash 0$ ')

$\mathrm{i}=\mathrm{n}+1$;

$s[-1]='$ ';

while (i--)

if $(*--s !=1)$

break;

$s[1]=' \backslash 0^{\prime} ;$

)

printf("8s $\backslash \mathrm{n} "$, str) ;

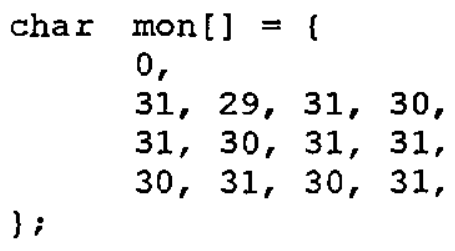


了

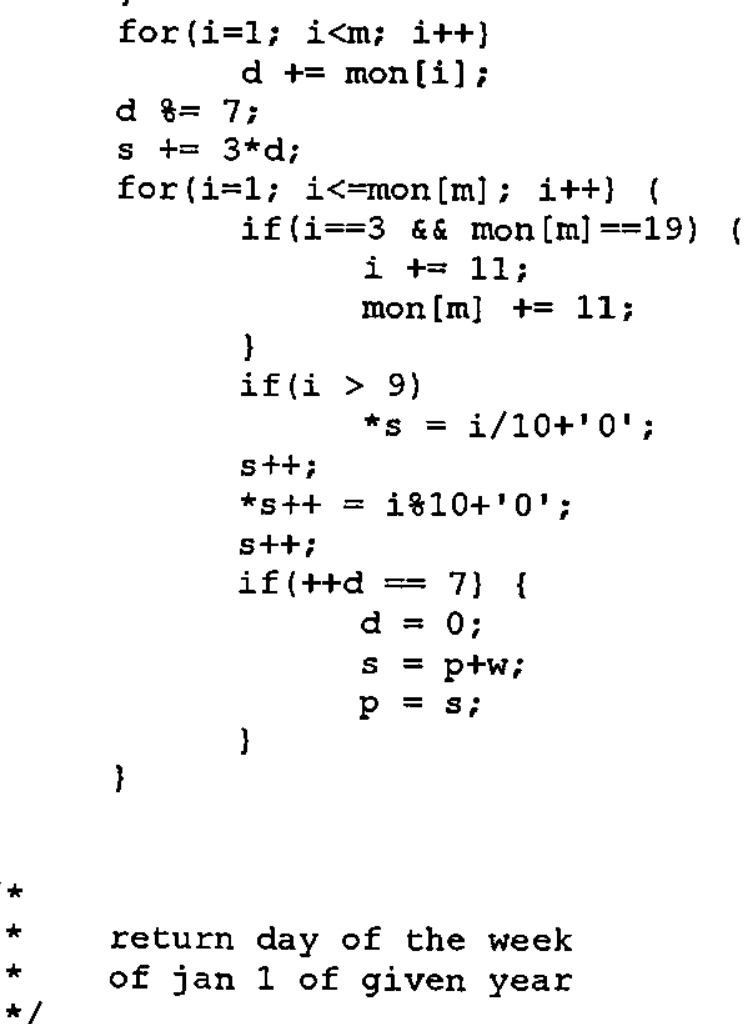




\section{Versōes Do Programa CAL}

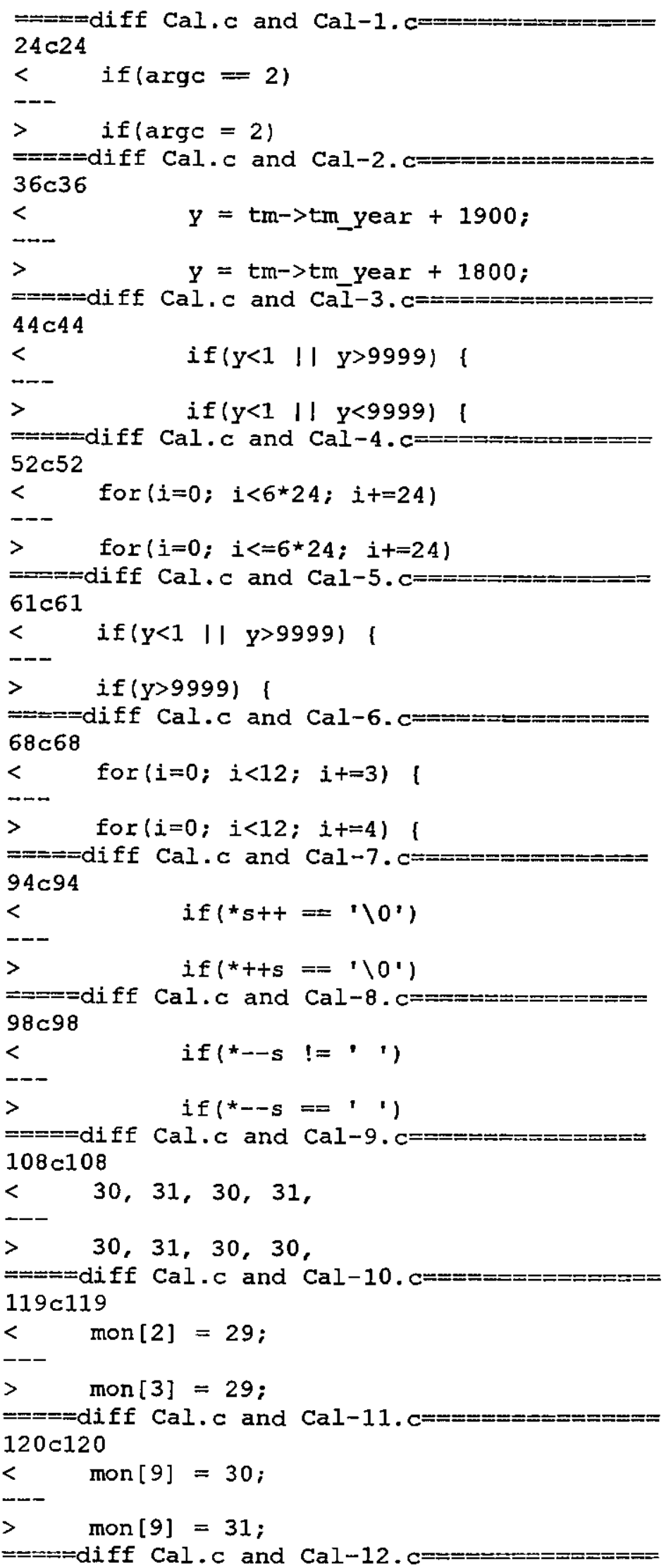

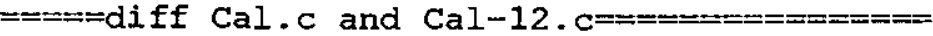
$127,129 \mathrm{~d} 126$ 


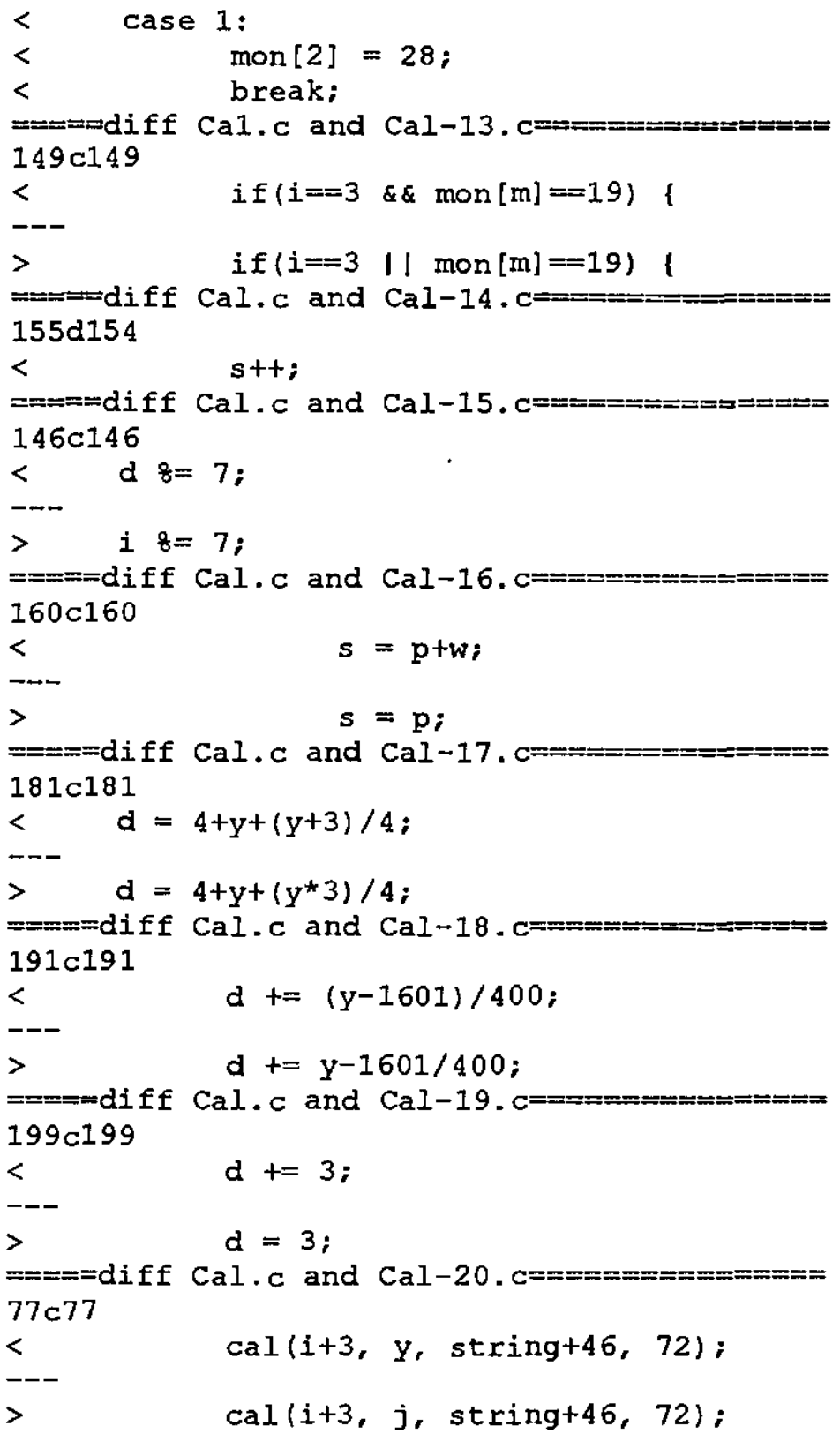

\section{Programa Checkeq}

$1 *$

* Copyright (c) 1987 Regents of the University of California.

* All rights reserved. The Berkeley software License Agreement

* specifies the terms and conditions for redistribution.

$\star /$

\#ifndef lint

char copyright [] =

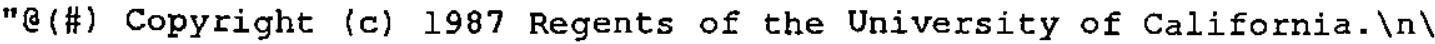
All rights reserved. $\mid \mathrm{n}^{\prime \prime}$; \#endif $/ *$ not lint $* /$

\#ifndef lint

static char sccsid[] = "[(\#) checkeq.c 4.3 (Berkeley) 12/2/87"; 


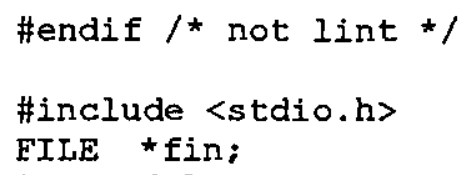




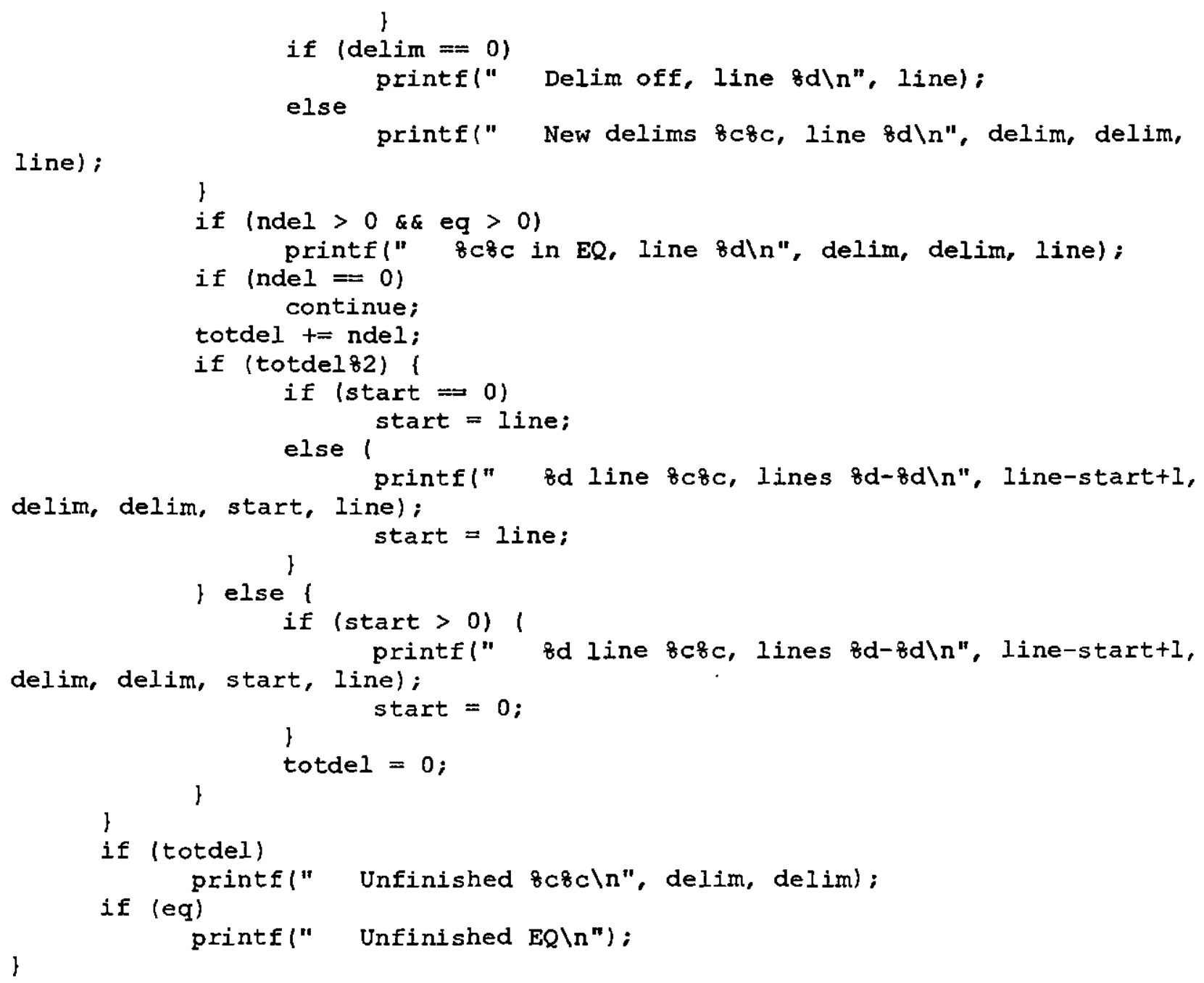

\section{Versőes Do Programa CHECKEQ}

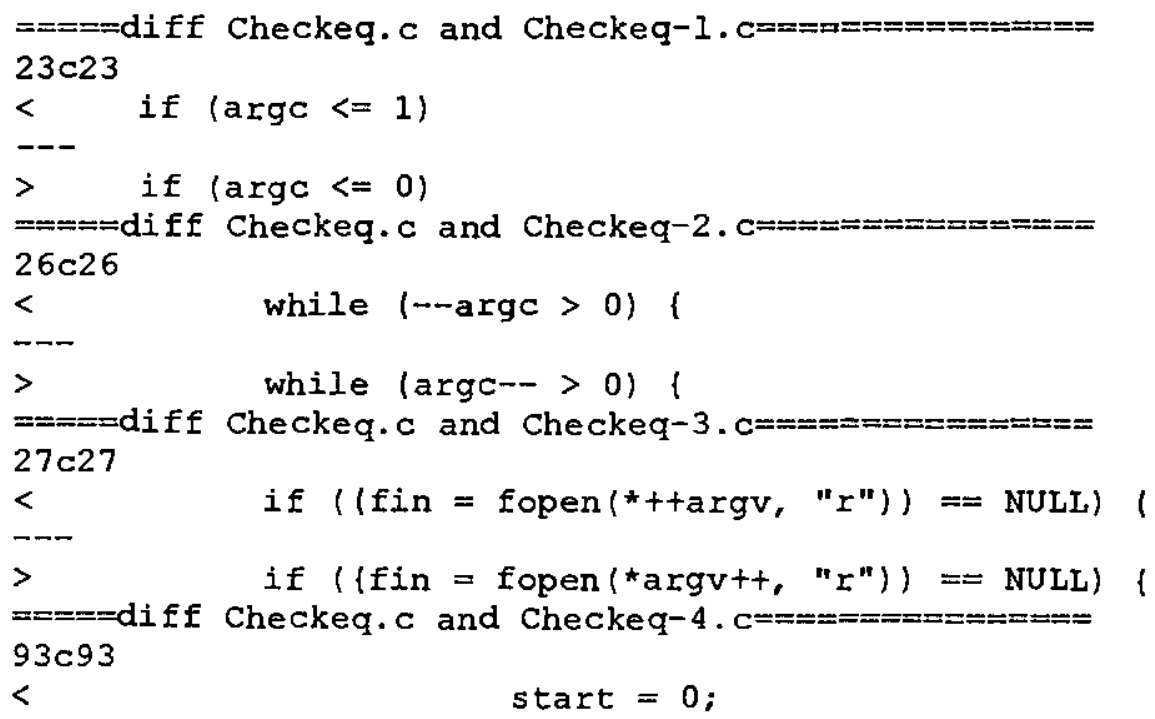


$>\quad$ totdel $=0$;

$====$ diff Checkeq. $c$ and Checkeq-5.c== $=====0=0==$

$44 c 44$

$<\quad$ start $=$ eq $=$ line $=$ ndel $=$ totdel $=0$;

$---$

$>\quad$ start $=$ line $=$ ndel $=$ totdel $=0$;

$====$ diff Checkeq. $\mathrm{C}$ and Checkeq-6. $\mathrm{c}=\mathrm{m}============$

$45 c 45$

$<$ while (fgets (in, 600, f) != NULL) ।

$>\quad$ while (fgets (in, 300, f) != NULL) (

$=====$ diff Checkeq. $c$ and Checkeq-7.c==:=:==:===== $47 \mathrm{c} 47$

$<\quad$ ndel $=0$;

$>\quad$ ndel $=1$;

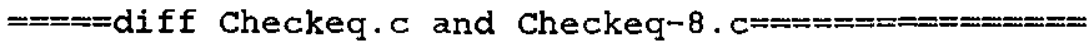
$50 c 50$

$<$ ndelt+;

-- -

$>\quad$ ndel--;

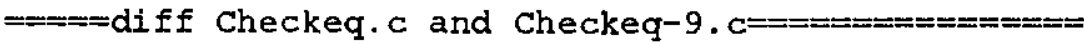
$95 \mathrm{~d} 94$

$<\quad$ totdel $=0$;

$====$ diff Checkeq. $c$ and Checkeq-10. $c============$ $51 \mathrm{c} 51$

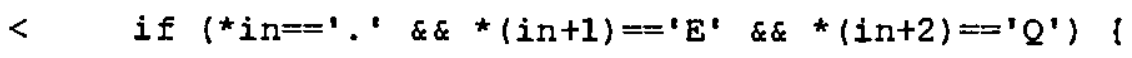

$-\cdots$

$>\quad$ if $\left(*^{*}{ }^{\prime}==^{\prime} .^{\prime} \& \& *^{*}(i n+1)==^{\prime} E^{\prime} \mid 1 *(i n+2)==^{\prime} Q^{\prime}\right)$

$====$ diff Checkeq. $c$ and Checkeq-11. c== $=========$ $56 c 56$

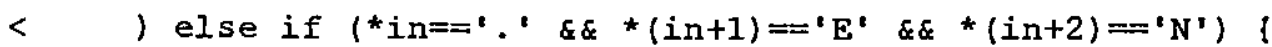

$-\cdots$

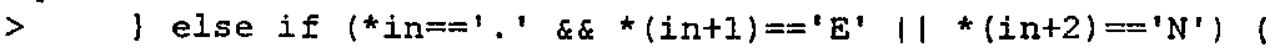

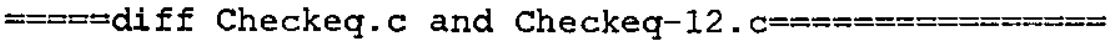
$60 c 60$

$<\quad$ eq $=0$;

$>\quad$ eq $=1$;

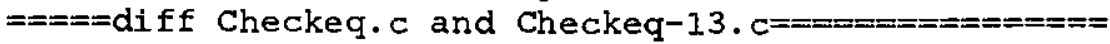
$64 c 64$

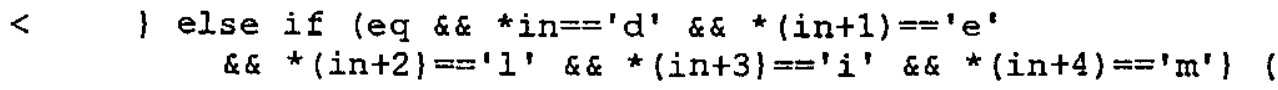




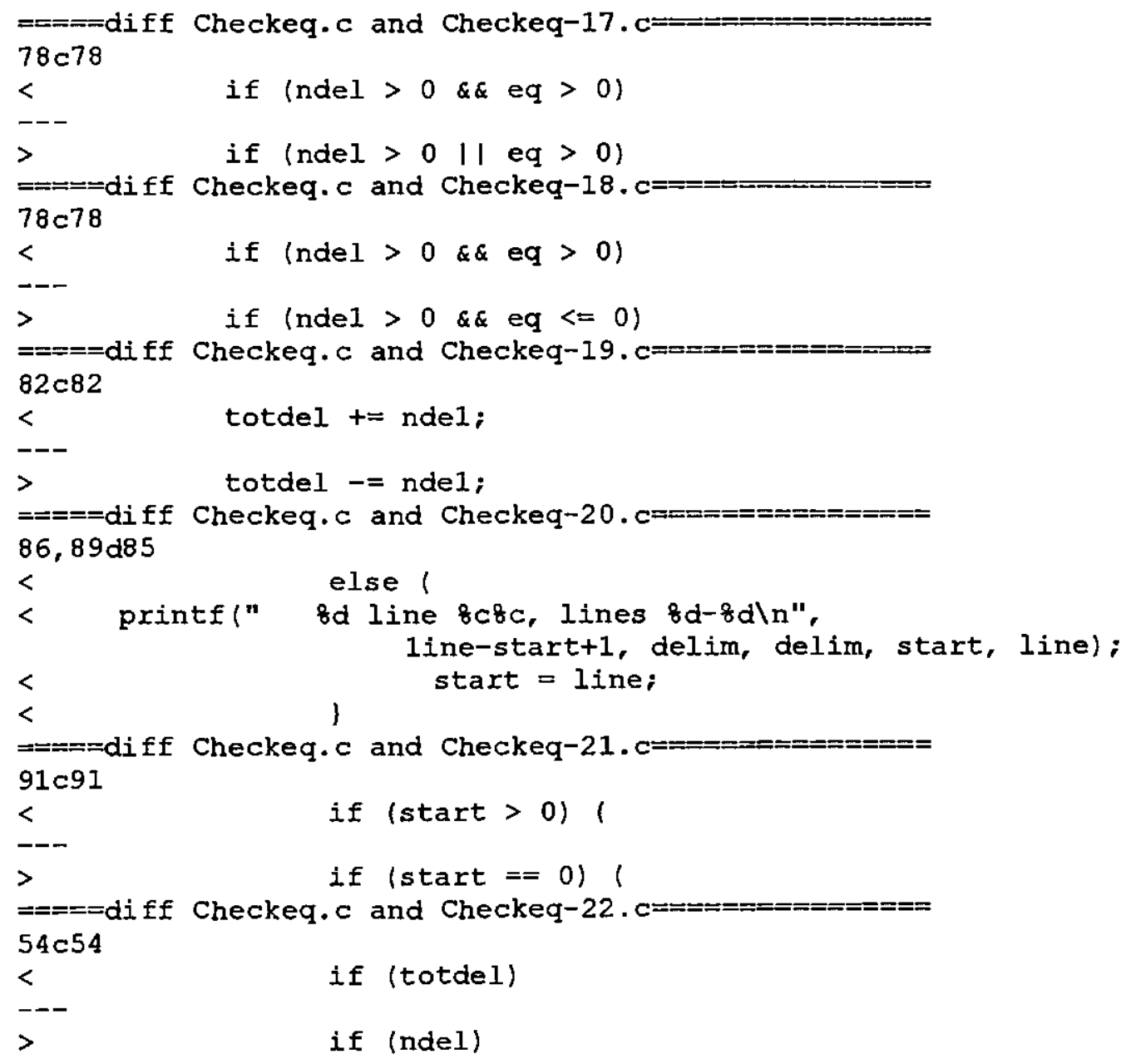

\section{Programa Comm}

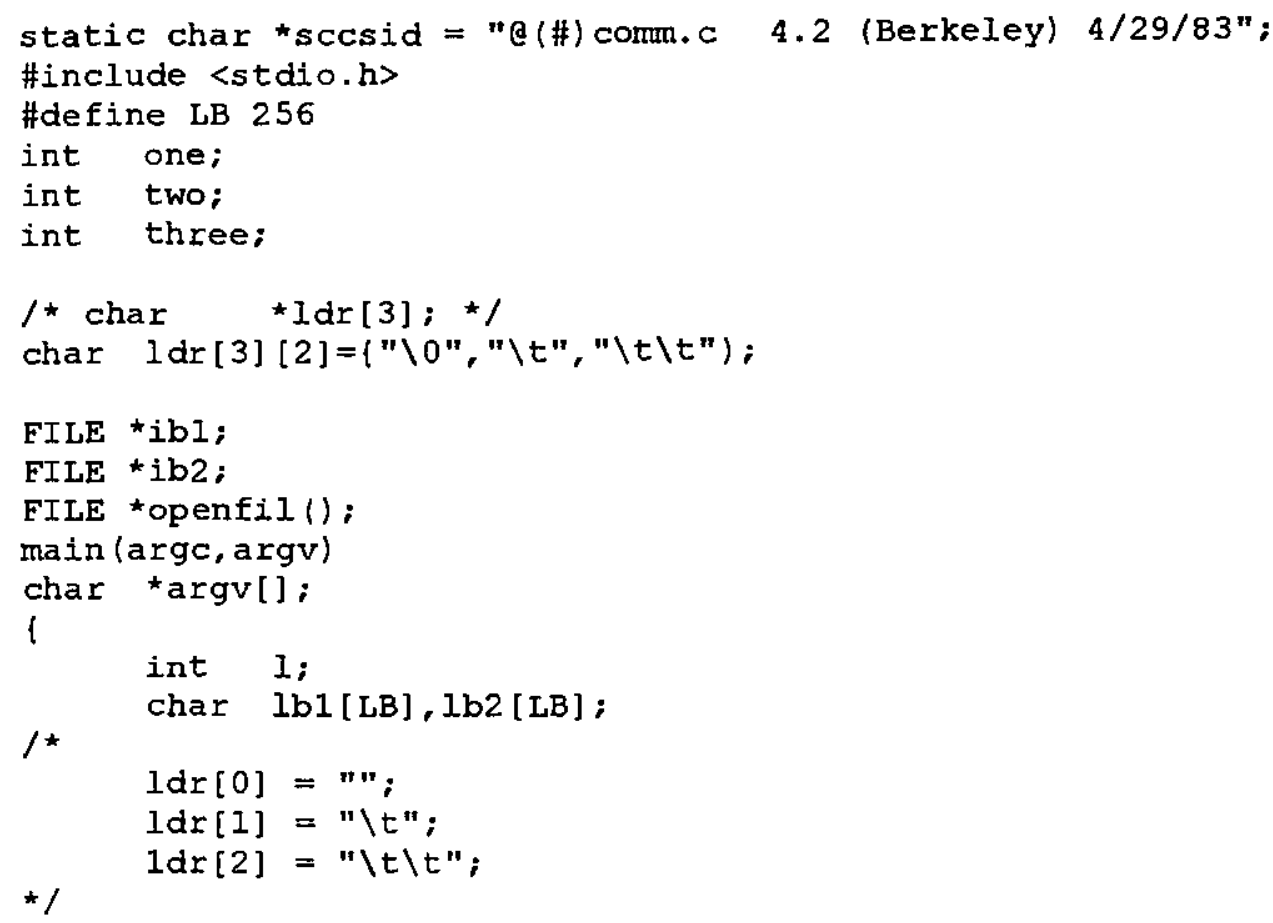




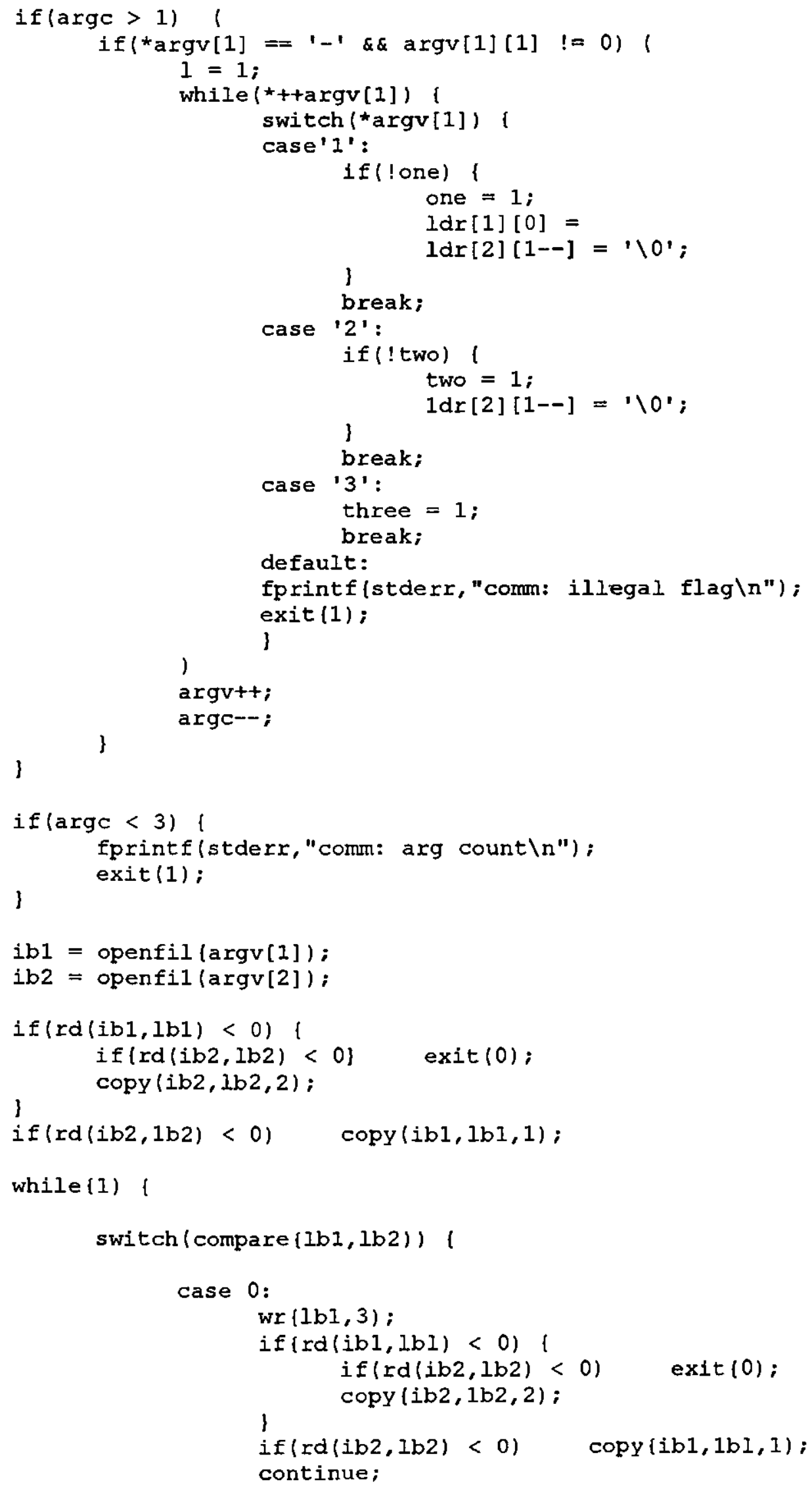




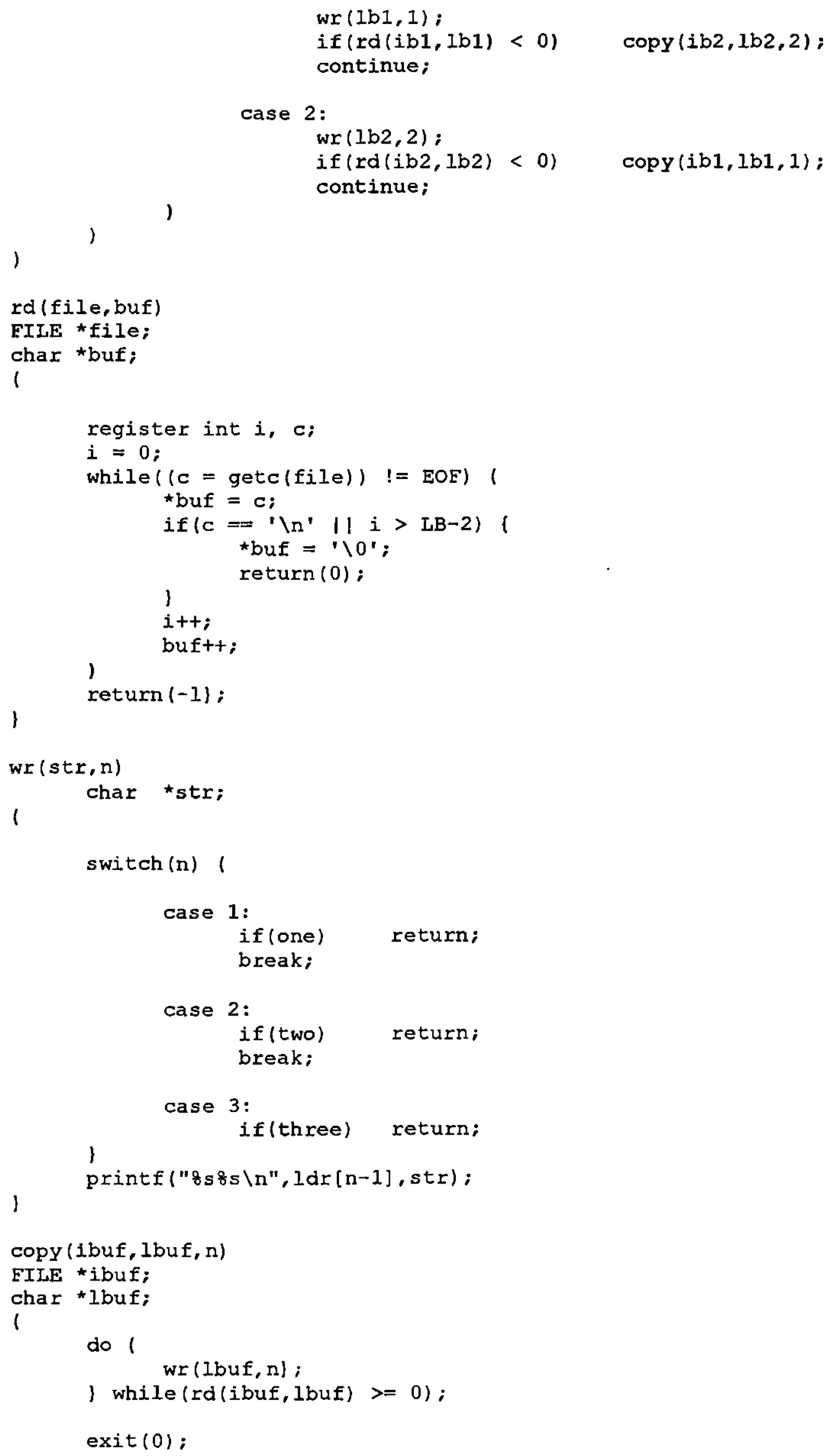




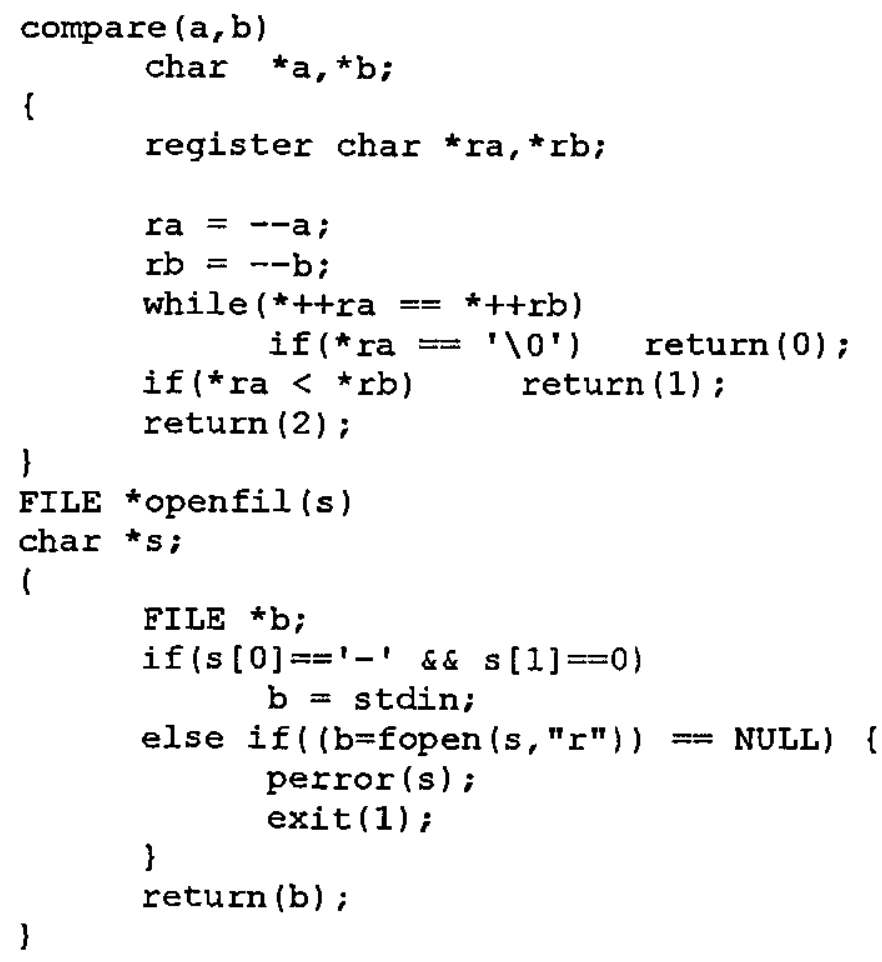

\section{Versões Do Programa COMM}

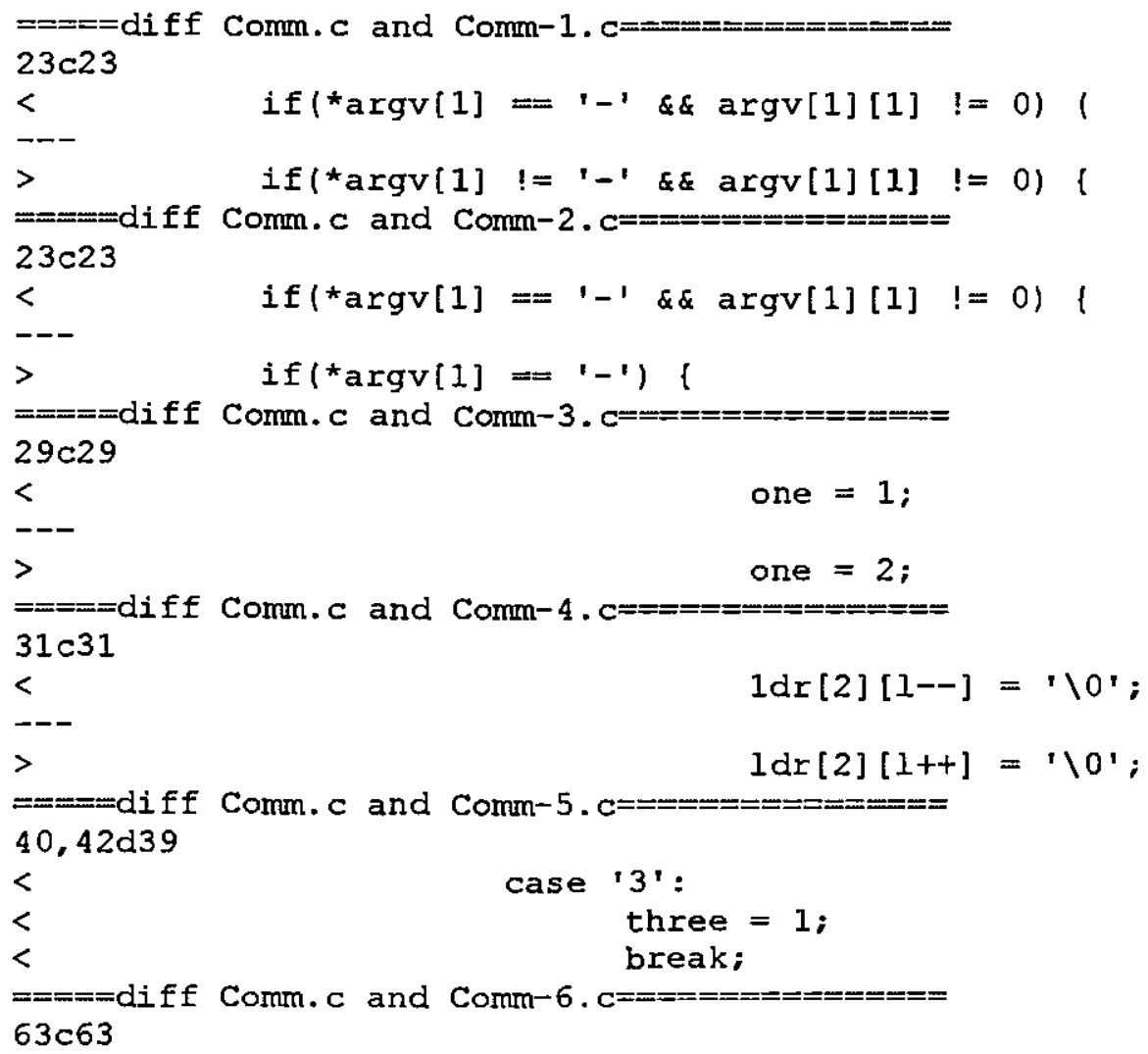




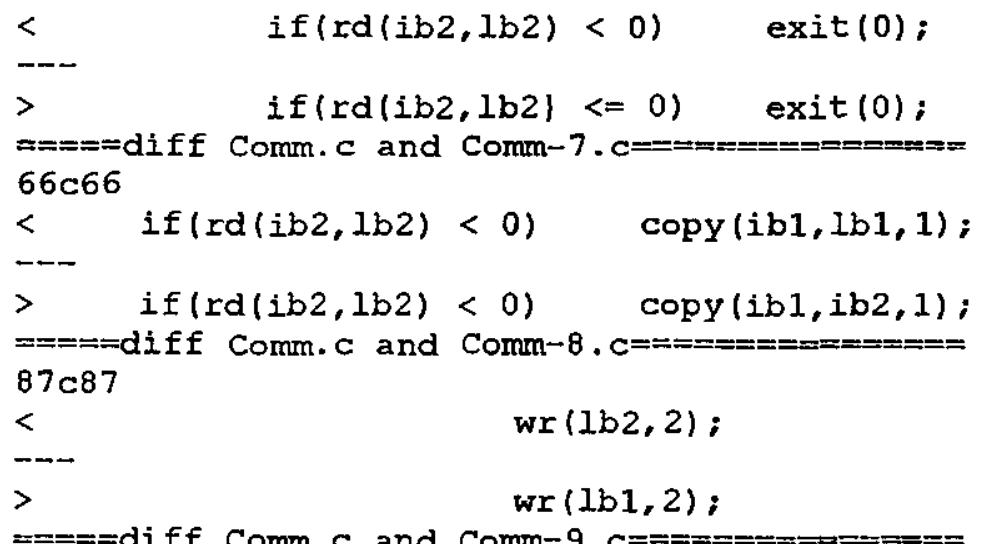

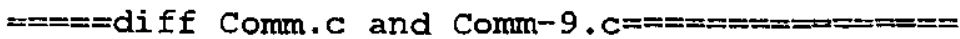
$103 \mathrm{c} 103$

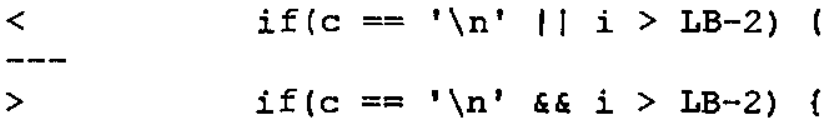

$===2$ diff Comm. $c$ and Comm-10. c $103 \times 103$

$<\quad$ if $(\mathrm{C}=\bar{\prime}=\mid \mathrm{n}$ ' || i $>$ LB-2) 1

$>\quad$ if $\left.\left(\mathrm{C}={ }^{\prime} \backslash \mathrm{n}^{\prime}|| \mathrm{i}\right\rangle=\mathrm{LB}-2\right) \mid$

$====d i f f$ Comm. $c$ and Comm-1I. $\mathrm{C}==$ $108 \mathrm{~d} 107$

$<$ buft+;

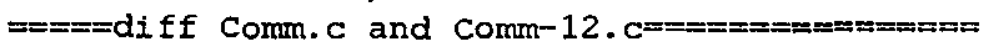
$12 \mathrm{Id} 120$

< break;

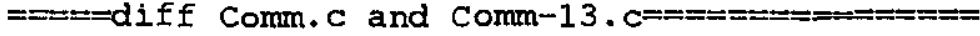
$127,128 \mathrm{~d} 126$

$<$ case 3:

$<$ if(three) return;

$=====$ diff Comm. $c$ and Comm-14. $c=============$ $139 c 139$

< \} while (rd(ibuf, lbuf\} >= 0);

$>\quad\}$ while(rd(ibuf,lbuf) $>0\}$;

$=====d i$ ff Comm. $c$ and Comm-15. $c====$ $151<151$

$<\quad$ while $(*++r a=*++r b)$

$>\quad$ while $(*++r a=* r b++)$

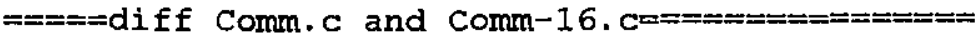
$160 \mathrm{c} 160$

$<\quad$ if $\left(s[0]==^{\prime}-'\right.$ \&\& $\left.s[I]==0\right)$

$--$

$>\quad$ if $\left(s[0]==^{\prime}-' \mid s[1]=0\right.$ )

$====$ diff Comm. $\mathrm{c}$ and Comm-17. $\mathrm{cm====}$ $160 c 160$

$<\quad$ if $\left(s[0]==^{\prime}-{ }^{\prime} \quad \& \& s[1]==0\right)$

$--\infty$

$>\quad$ if $(s[1]==0)$

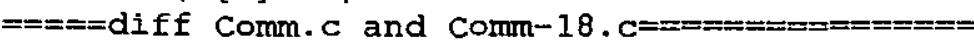
$153 \mathrm{c} 153$

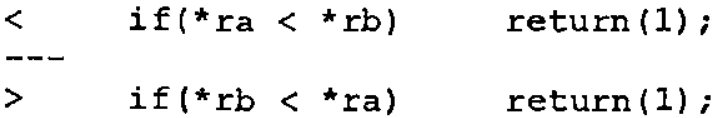

$=====$ diff Comm. $c$ and comm-19. $c==============$ $76 c 76$ 
copy $(\mathrm{ib} 2,1 \mathrm{~b} 2,2)$;

$---$

copy $(i b 1,1 b 1,2)$;

\section{Programa Look}

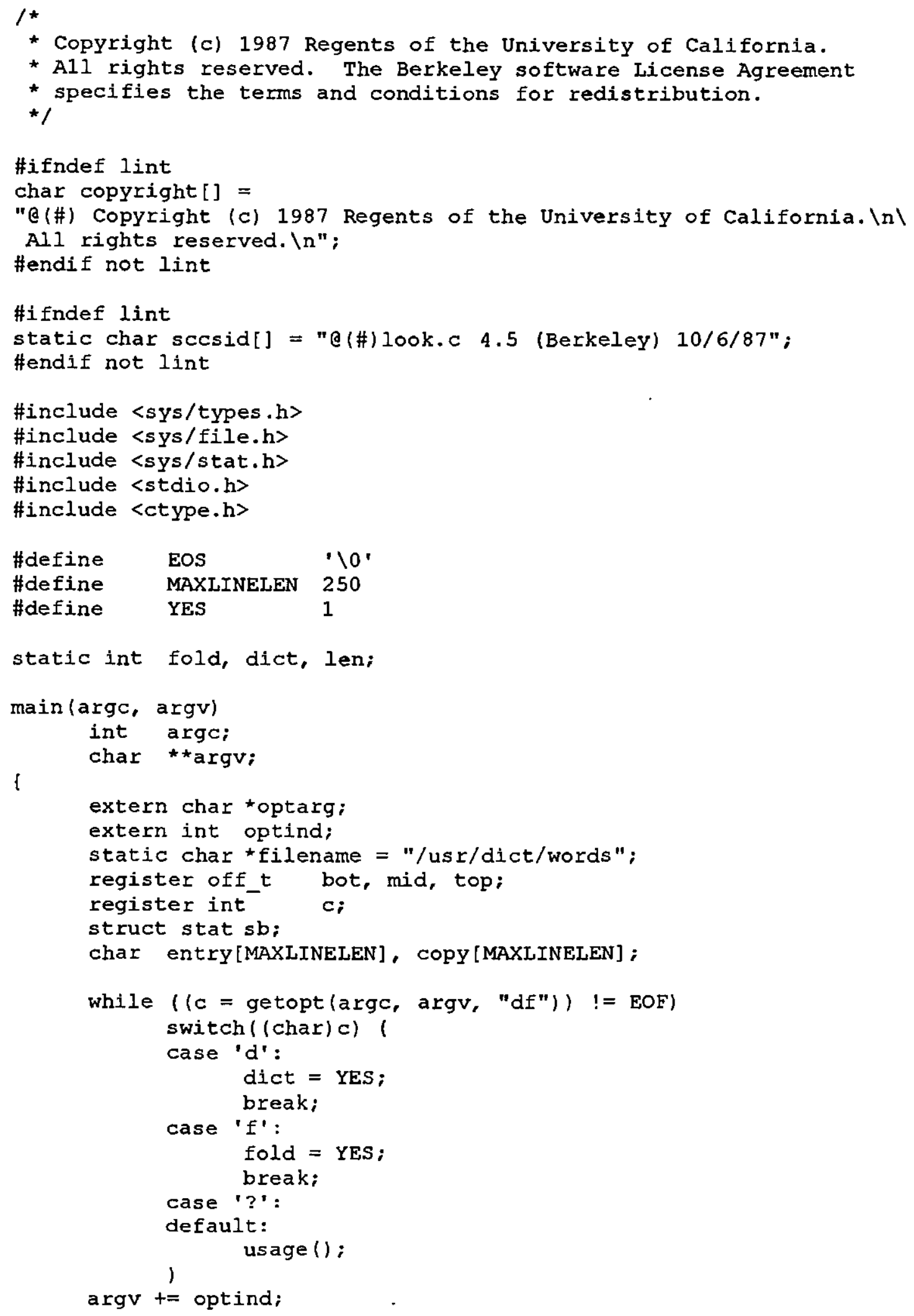




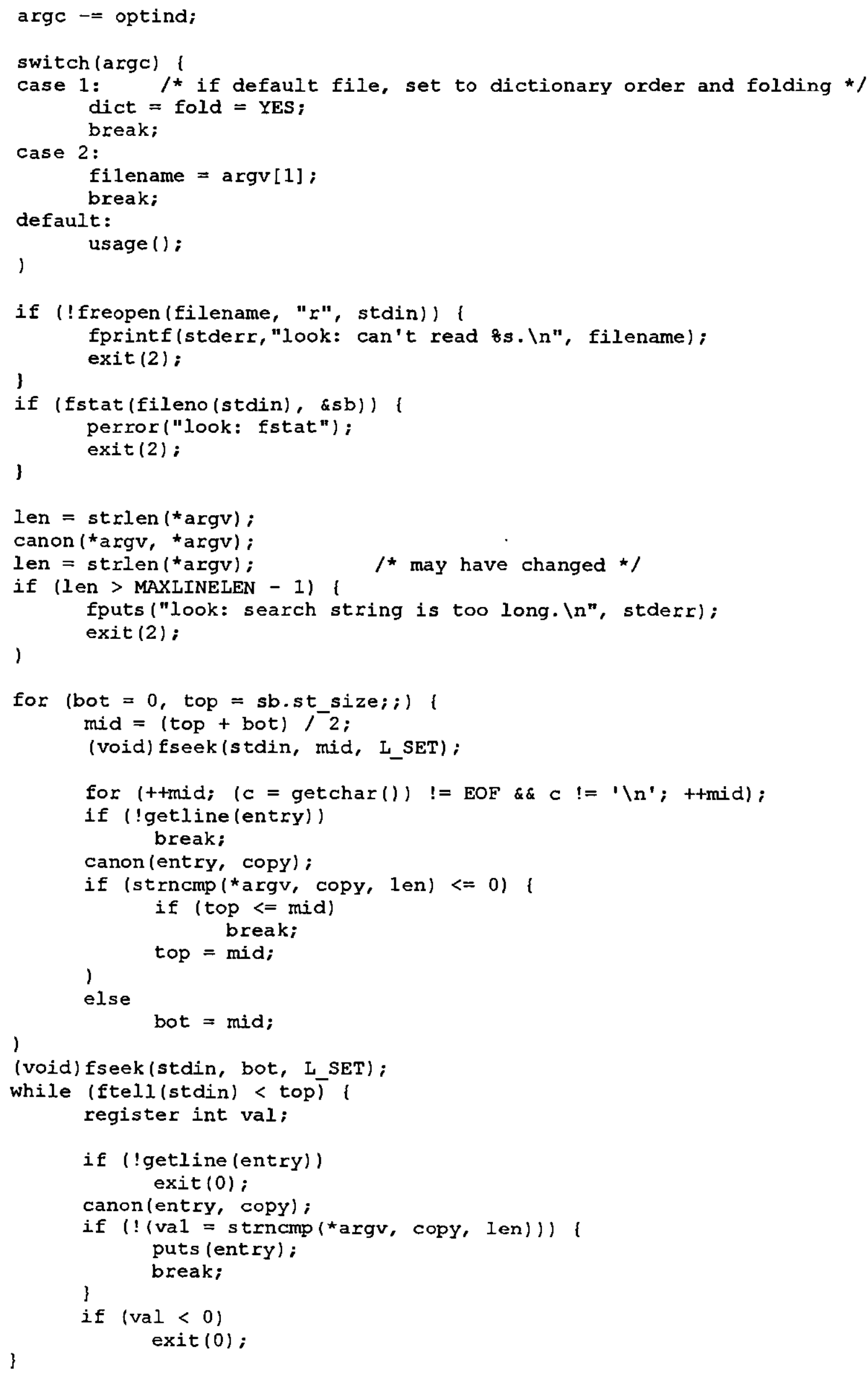




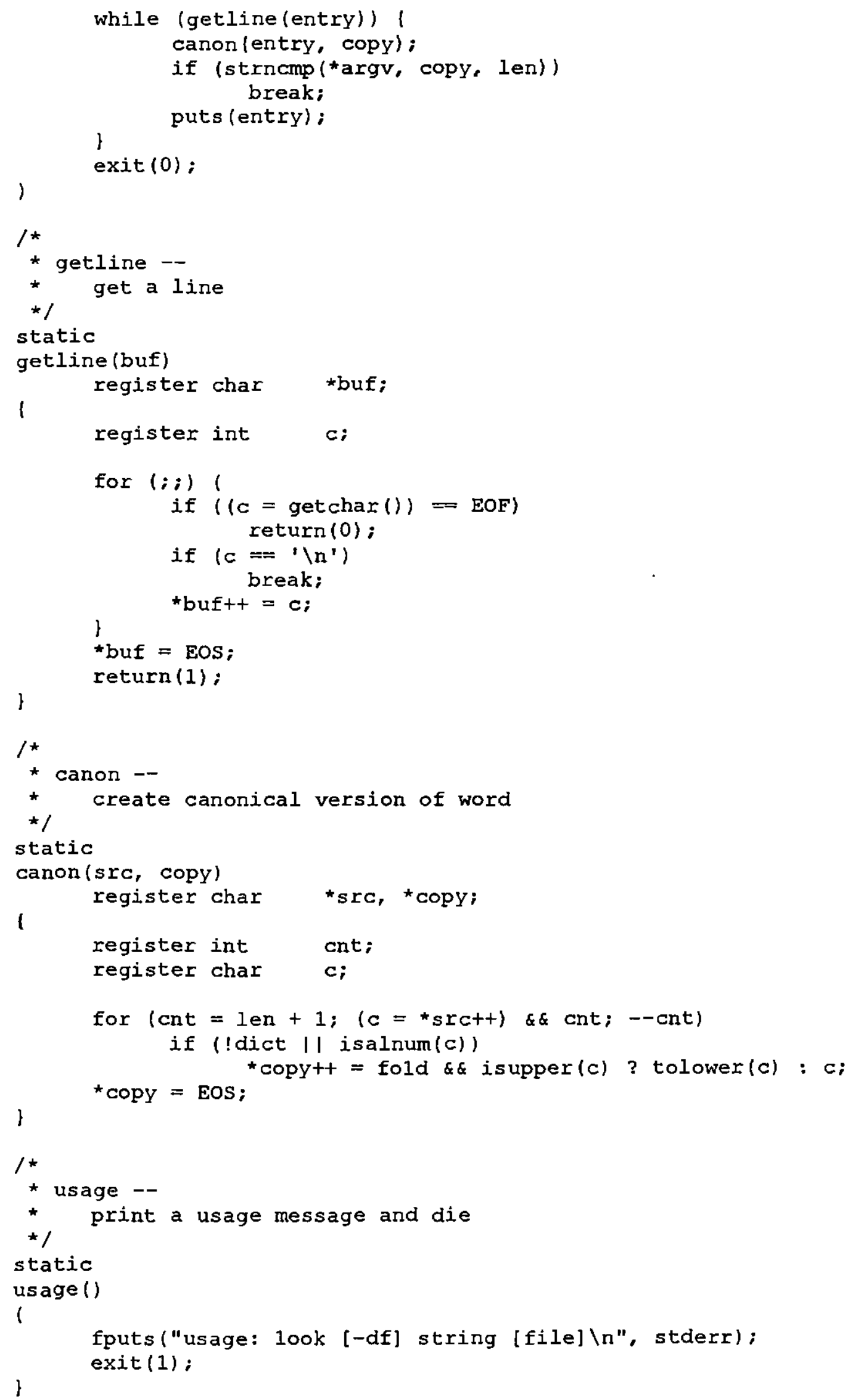




\section{Versões Do Programa LOOK}

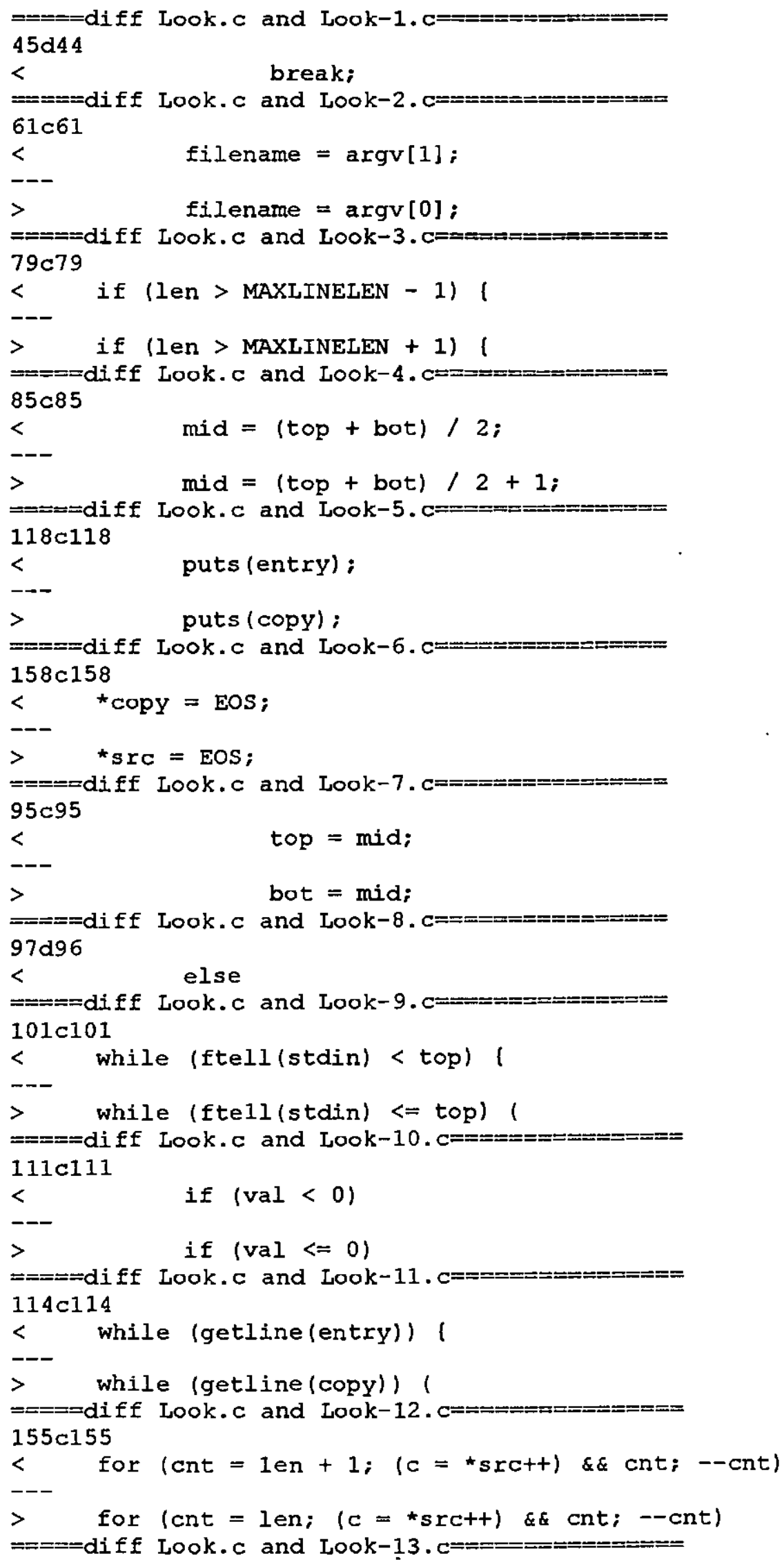


$138 \mathrm{c} 138$

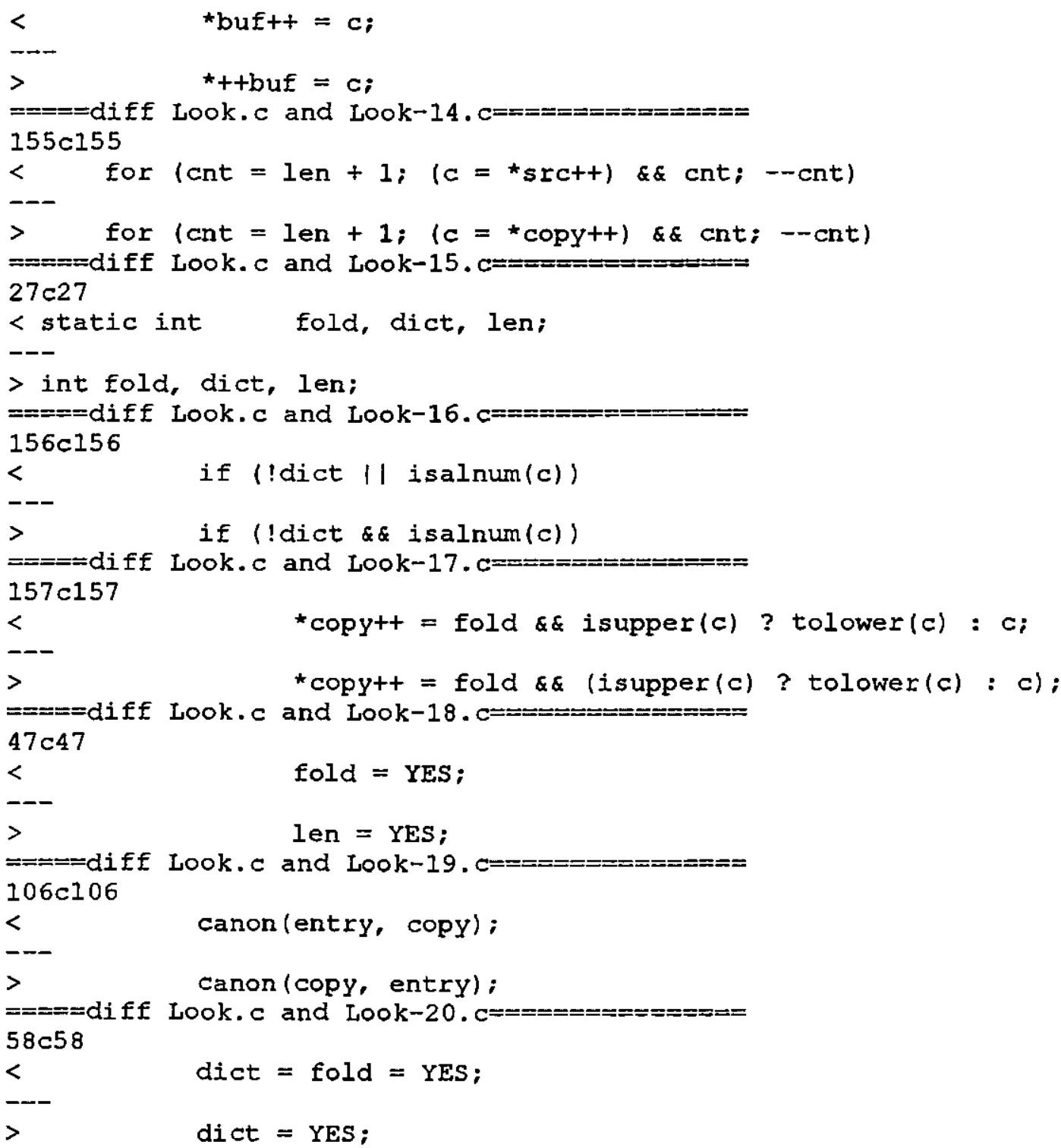

\section{Programa Uniq}

static char *sccsid $=$ "@(\#) uniq.c 4.1 (Berkeley) 10/1/80";

$1 *$

* Deal with duplicated lines in a file

$\star 1$

\#include <stdio.h>

\#include <ctype.h>

int fields;

int letters;

int linec;

char mode;

int uniq;

char *skip();

main (argc, argv)

int argc;

char *argv[]; 


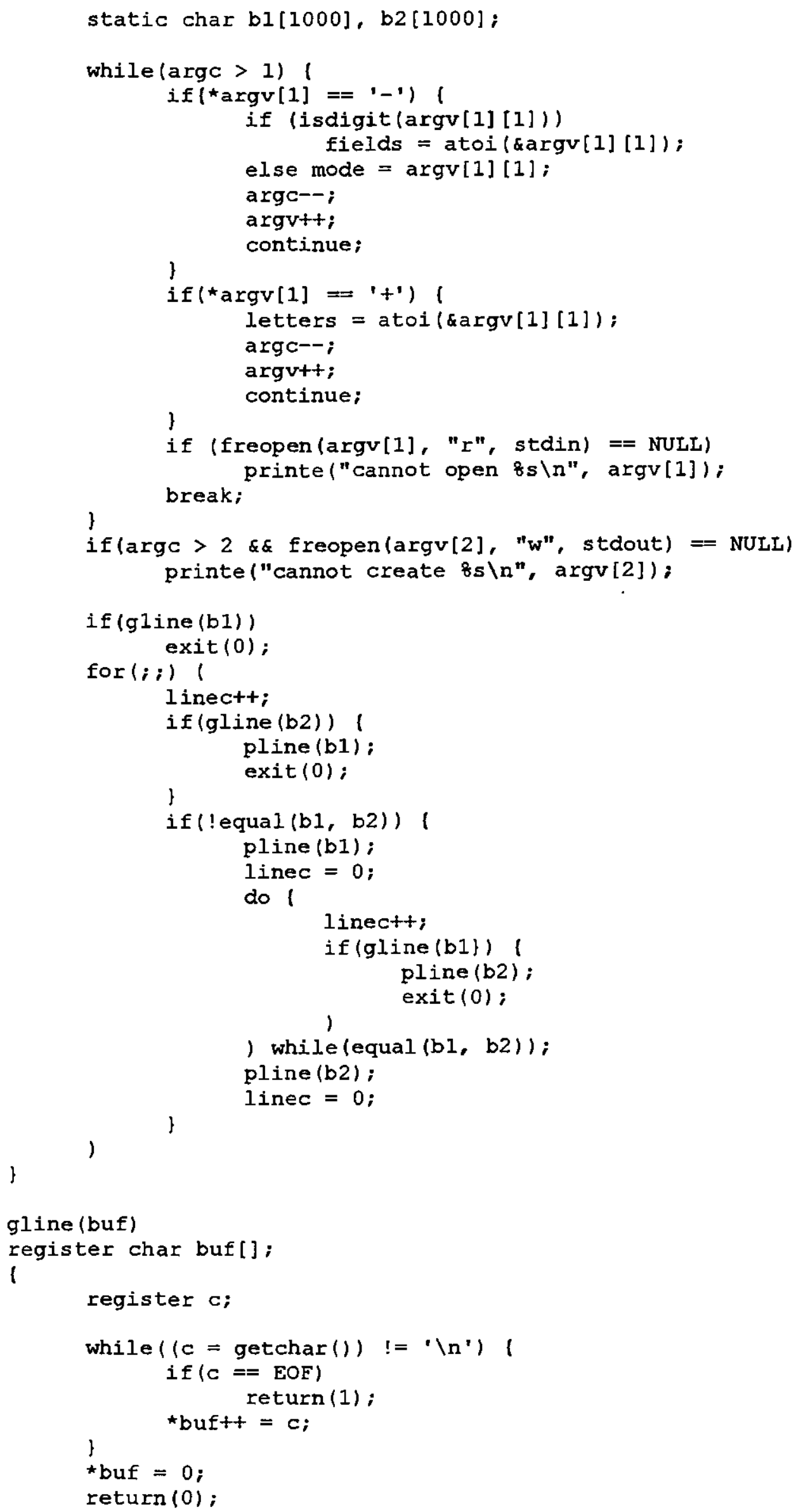


pline (buf)

register char buf[];

\{

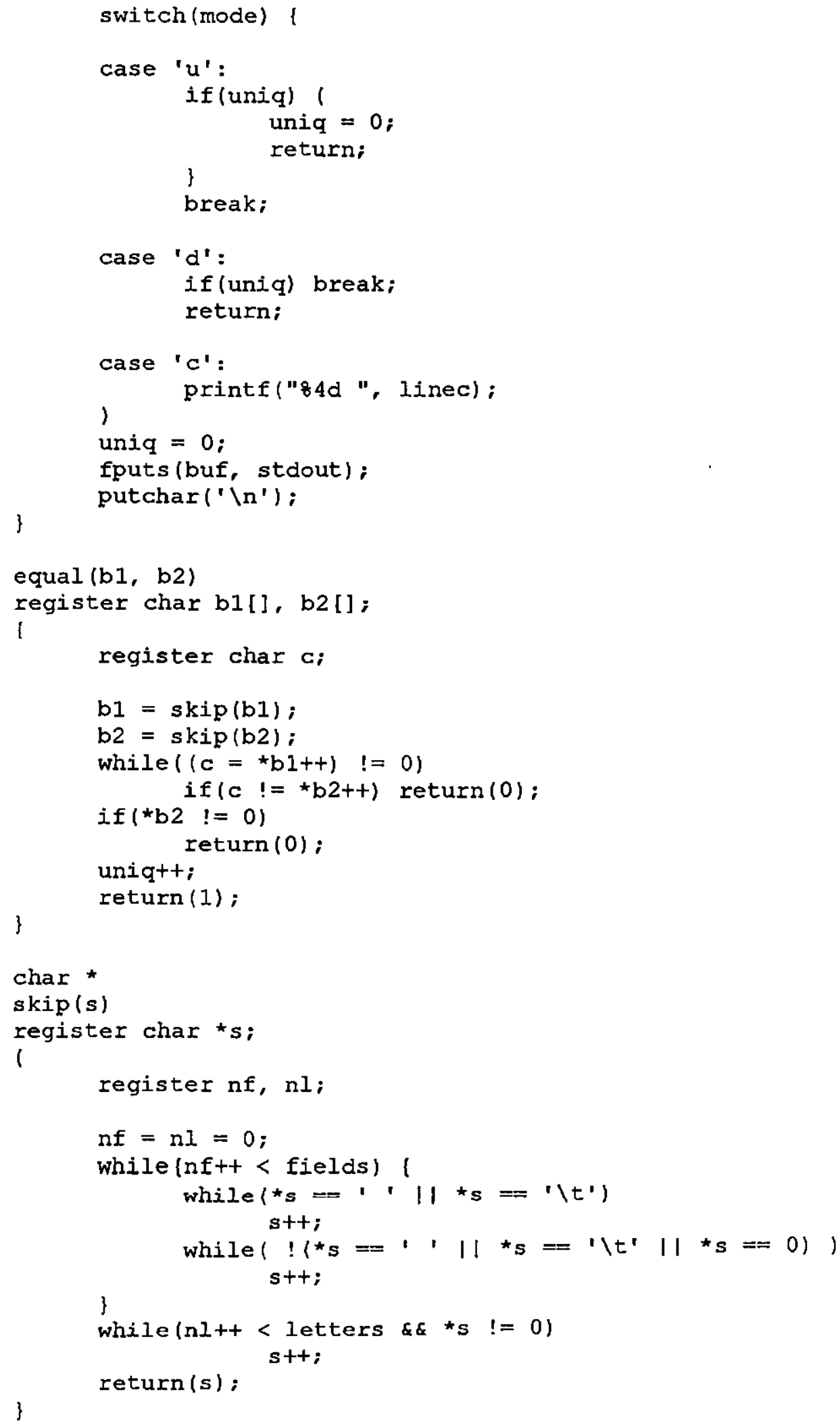


printe $(p, s)$

char ${ }^{*} p,{ }^{*}$;

(

fprintf(stderr, $p, s)$;

r exit (1):

\section{Versões Do Programa UNIQ}

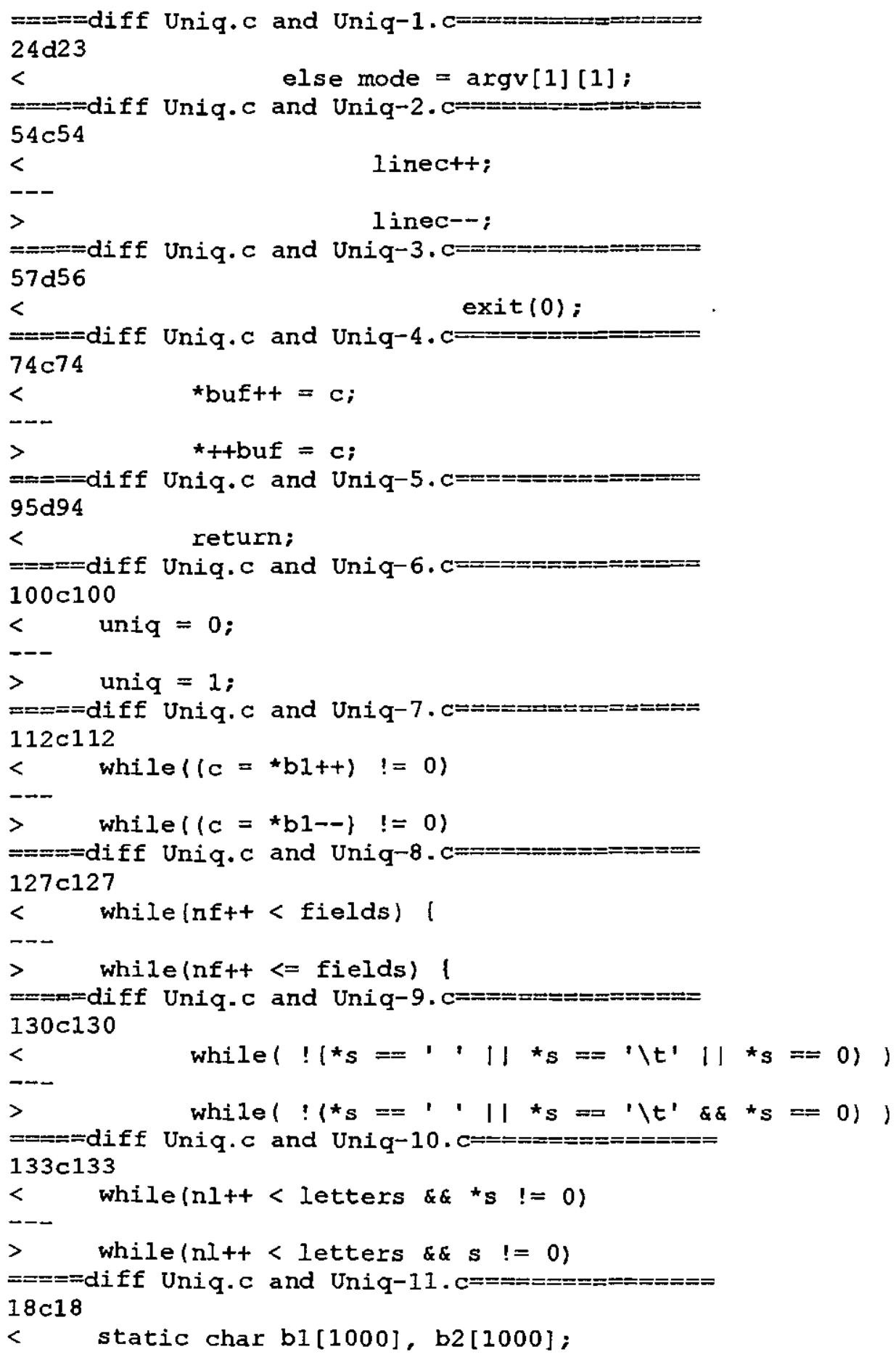


char b1[1000], b2 [1000];

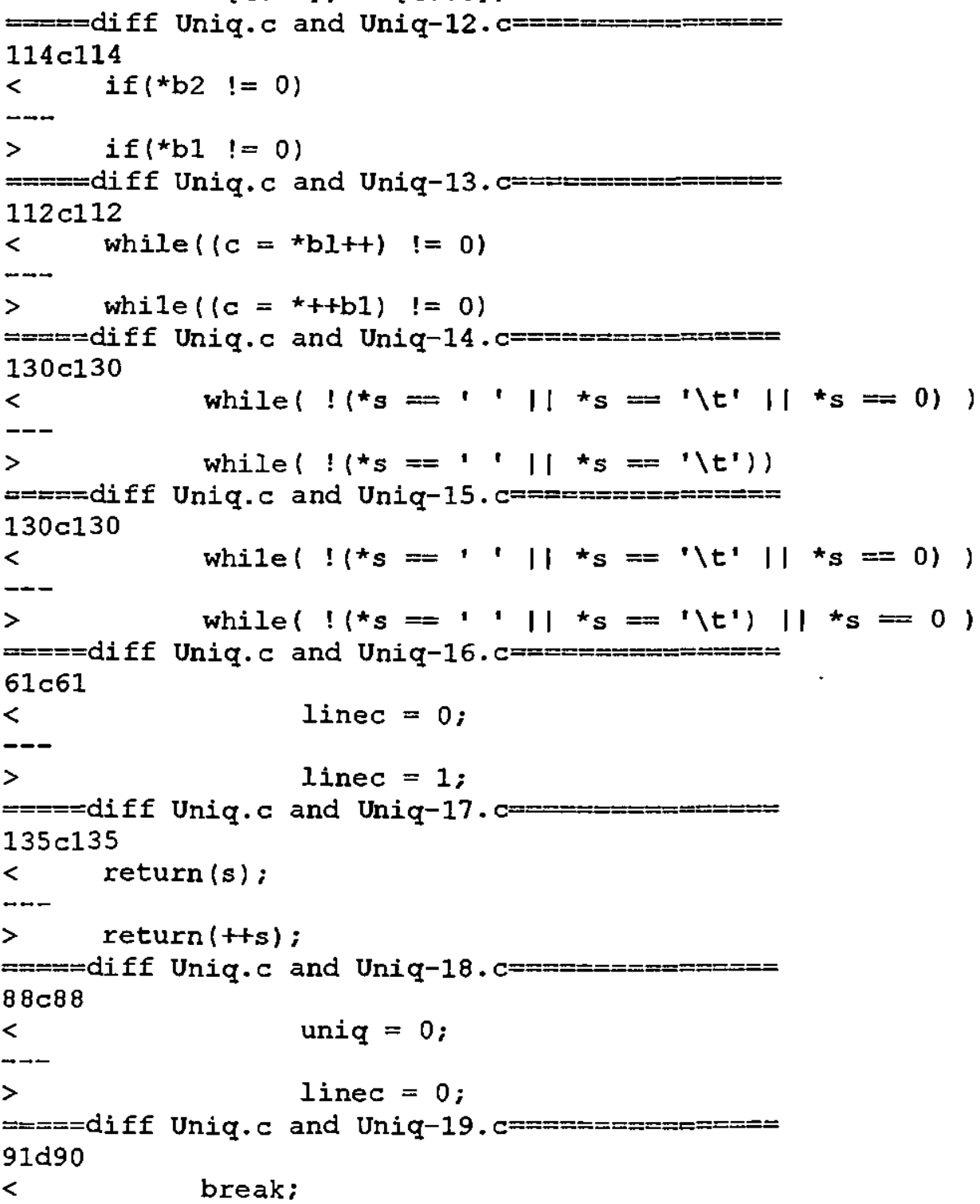




\section{Processo de Ativaçăo do erros do Programa SPACE}

Para gerar os programas com erros utilizou-se uma versão do SPACE adequada para essa atividade (prepro.c) utilizando-se o seguinte comando de compilação:

gcc prepro.c-DESF $\rightarrow$ SPROG.exe $-\mathrm{Im}-\mathrm{w}$

no qual:

prepro.c: programa fonte que ativa os erros

-DESF: primitiva que defini qual erro deve ser ativado; $\$ F=$ erro ativado 